\title{
O Teorema de Kronecker-Weber segundo Kronecker e Weber
}

Carlos Juiti Watanabe

\author{
DISSERTAÇÃO APRESENTADA \\ $\mathrm{AO}$ \\ INSTITUTO DE MATEMÁTICA E ESTATÍSTICA \\ DA \\ UNIVERSIDADE DE SÃO PAULO \\ PARA \\ OBTENÇÃO DO GRAU \\ $\mathrm{DE}$ \\ MESTRE EM MATEMÁTICA
}

Orientador: Prof. Dr. Paulo Agozzini Martin

14 de Julho de 1997 


\section{O Teorema de Kronecker-Weber segundo Kronecker e Weber}

Este exemplar corresponde à redação final da dissertação, devidamente corrigida, apresentada por Carlos Juiti Watanabe e aprovada pela comissão julgadora.

São Paulo, julho de 1997.

Banca examinadora:

Prof. Dr. Paulo Agozzini Martin

IME-USP

Prof. Dr. Ricardo Bianconi

IME-USP

Prof. Dr. Michel Spira

UFMG 


\section{Índice}

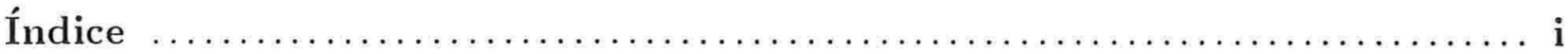

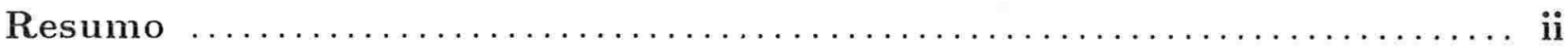

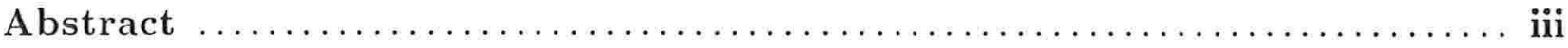

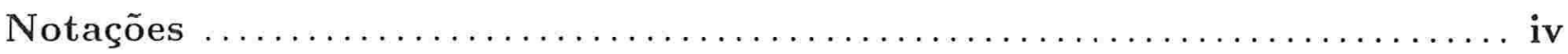

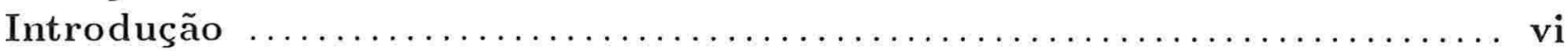

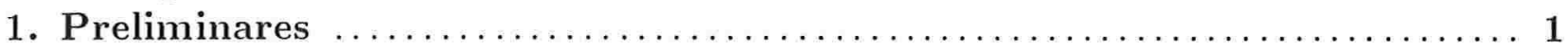

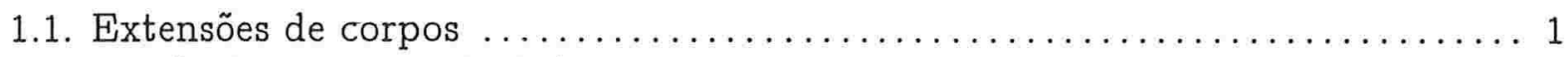

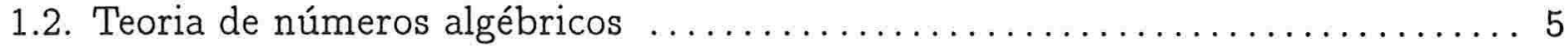

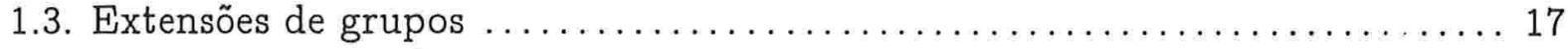

2. Alguns Teoremas Sobre Extensões Centrais $\ldots \ldots \ldots \ldots \ldots \ldots \ldots \ldots \ldots \ldots$

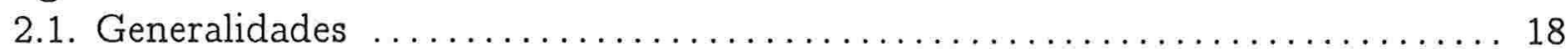

3. Alguns Resultados Sobre Corpos Ciclotômicos $\ldots \ldots \ldots \ldots \ldots \ldots \ldots \ldots$

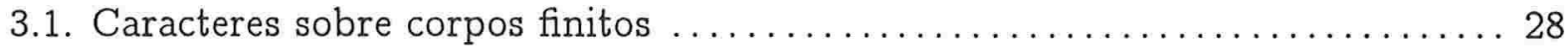

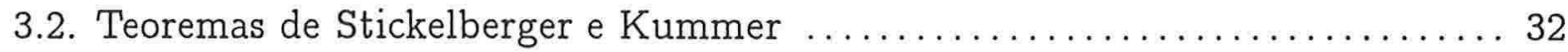

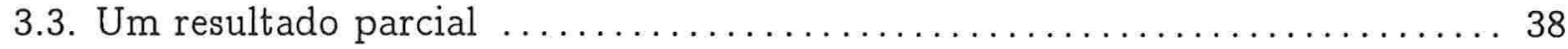

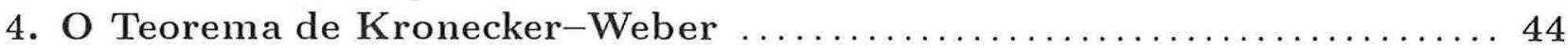

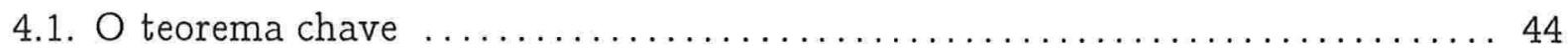

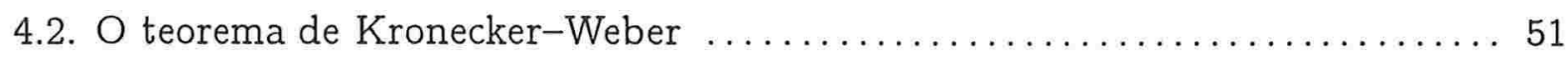

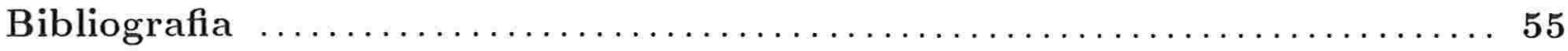




\section{Resumo}

Este texto destina-se a demonstrar o teorema de Kronecker-Weber, que afirma que toda extensão abeliana de $\mathbb{Q}$ é ciclotômica.

Existem muitas demonstrações do célebre teorema, e baseadas sobre princípios diversos - com maior ou menor necessidade de resultados tirados da teoria algébrica dos números.

O objetivo deste trabalho é apresentar uma demonstração desse resultado seguindo de perto as demonstrações originais de Kronecker e de Weber.

Embora seja mais longa e mais elaborada do que as provas mais modernas (como por exemplo de Hilbert ou Šafarevič), baseia-se sobre princípios muito gerais, que podem eventualmente prestar-se a generalizações em outros contextos. 


\section{Abstract}

This work aims to prove the Kronecker-Weber theorem, which asserts that every abelian extension of $\mathbb{Q}$ is ciclotomic.

There are many proofs of this famous theorem, all of them based on several principles which requires more or less results of algebraic number theory.

The aim of this work is to present one proof of this result as close as possible the original proof of Kronecker and Weber.

This proof is longer and more elaborated than modern ones, like Šafarevič's and Hilbert's, but it is based in general principles and it might be generalized in other contexts. 


\section{Notações}

Neste texto, utilizaremos várias notações, que, para maior facilidade do leitor, serão listadas abaixo:

$\begin{aligned} \mathbb{Q} & \text { o corpo dos números racionais; } \\ \operatorname{Gal}(\mathbb{L} / \mathbb{K}) & \text { o grupo de Galois da extensão de corpos } \mathbb{L} / \mathbb{K} ; \\ \mathbb{Z}(G) & \text { o centro do grupo } G \text { : } \\ \mathbb{R} & \text { o corpo dos números reais; } \\ \mathbb{C} & \text { o corpo dos números complexos; } \\ \mathbb{K} & \text { o fecho algébrico do corpo } \mathbb{K}: \\ \mathbb{L}^{H} & \text { o corpo fixo por } H \text { em } \mathbb{L}: \\ \mathbb{Z} & \text { o anel de inteiros racionais: } \\ \operatorname{Aut}(H) & \text { o grupo dos automorfismos do grupo } H ; \\ \mathcal{O}_{\mathbb{L}} & \text { o anel de inteiros do corpo } \mathbb{L} ; \\ I_{\mathbb{L}} & \text { o grupo dos ideais fracionários de } \mathbb{L} ; \\ P_{\mathbb{L}} & \text { o grupo dos ideais principais de } \mathbb{L} ; \\ |, \mathfrak{a}| \mathfrak{b} & \text { o símbolo de divisão, a divide } \mathfrak{b} ; \\ \mathbb{N} & \text { o conjunto dos números naturais; } \\ \mathcal{O}_{\mathbb{L}}\left[\mathbb{X}^{\prime}\right] & \text { o anel de polinômios com coeficientes em } \mathcal{O}_{\mathbb{L}} ; \\ v_{\mathfrak{p}} & \text { a valorização induzida pelo ideal } \mathfrak{p} ; \\ N_{\mathbb{L} / \mathbb{F}} & \text { a norma relativa de } \mathbb{L} \text { sobre } \mathbb{K} ; \\ \mathbb{Z}[X] & \text { o anel dos polinômios com coeficientes em } \mathbb{Z} ; \\ \epsilon(\mathfrak{P} / \mathfrak{p}), \epsilon_{i} & \text { o índice de ramificação de } \mathfrak{P} ; \\ f(\mathfrak{P} / \mathfrak{p}), f_{i} & \text { o grau do ideal primo } \mathfrak{P} ; \\ \mathcal{O}_{\mathbb{L} v} & \text { o anel de valorização de } v ; \\ P_{v} & \text { o ideal de valorização de } v ; \\ C_{n} & \text { o corpo totalmente real de grau } 2^{n} ; \\ \mathbb{Z}[G] & \text { o anel de grupos de } G ; \\ T f & \text { a transformada de Fourier de } f ; \\ I^{-1} & \text { o inverso do ideal fracionário } I ; \\ A \backslash B & \text { a diferença dos conjuntos } A \text { e } B ;\end{aligned}$




$$
\begin{array}{r}
T_{\mathfrak{P} / \mathfrak{p}}, T(\mathfrak{P} / \mathfrak{p}) . T \\
D_{\mathfrak{P} / \mathfrak{p}}, D(\mathfrak{P} / \mathfrak{p}) . D \\
D_{\mathfrak{p}} \\
\mathfrak{D}_{\mathbb{L} / \mathbb{R}} \\
\mathfrak{D}_{\mathbb{L} / \mathbb{R}}(\mathfrak{a}) \\
\operatorname{Tr}_{\mathbb{L} / \mathbb{Q}}(x), T r(x) \\
\mu_{m} \\
\operatorname{ker}(\alpha) \\
p \\
\mathbb{F}_{p} \\
\mathbb{F}^{*} \\
m \\
\zeta_{m} \\
\mathbb{Q}^{\prime} \\
\mathbb{F}_{q} \\
\mu_{n}=\left\{\zeta \in \mathbb{C}_{n}=\zeta^{n}=1\right\} \\
\lambda: \mathbb{F}_{q} \longrightarrow \mu_{p} \\
\chi: \mathbb{F}_{q}^{*} \mu_{q-1} \\
G \\
r: G \longrightarrow\left(\mathbb{Z} / m \mathbb{Z}^{*}\right.
\end{array}
$$

o grupo de inércia de $\mathfrak{P}$ sobre $\mathfrak{p}$;

o grupo de decomposição de $\mathfrak{P}$ sobre $\mathfrak{p}$ :

o grupo de decomposição de $\mathfrak{p}$ sobre $p$;

o diferente da extensão de $\mathbb{L} / \mathbb{K}$;

o diferente da extensão do ideal a:

o traço de $x$ em relação a $\mathbb{L} / \mathbb{Q}$;

o grupo das raízes $m$-ésimas da unidade;

o núcleo do morfismo $\alpha$;

um primo racional positivo;

o corpo primo de característica $p$;

o grupo dos elementos invertíveis de $\mathbb{F}$;

um inteiro racional positivo;

uma raiz $m$-ésima primitiva da unidade;

a extensão ciclotômica $\mathbb{Q}\left(\zeta_{m}\right)$;

um corpo finito com $q$ elementos, $q=p^{n}$;

o grupo das raízes $n$-ésimas da unidade;

o caracter de $\mathbb{F}_{q}$ dado por $\lambda(x)=\zeta_{p}^{T r(x)}$;

um caracter multiplicativo;

o grupo de Galois de $\mathbb{Q}^{\prime}$ sobre $\mathbb{Q}$;

o isomorfismo de grupos dado por $\sigma\left(\zeta_{m}\right)=\zeta_{m}^{r(\sigma)}$;

o grupo das unidades de $\mathbb{Q}\left(\zeta_{m}\right)$. 


\section{Introdução}

O teorema de Kronecker-Weber afirma: se $\mathbb{L} / \mathbb{Q}$ é uma extensão galoisiana finita, cujo grupo de Galois é abeliano, então existe uma raiz da unidade $\zeta$ tal que $\mathbb{L} \subset \mathbb{Q}(\zeta)$.

Existem muitas demonstrações desse teorema, várias das quais utilizam mais resultados de teoria de números algébricos e da teoria de ramificação do que a apresentada aqui. Podemos citar, como exemplo a demonstração elaborada por Hilbert, que reduz o problema ao caso de extensões abelianas finitas de corpos $p$-ádicos, refazendo a teoria de Kummer para extensões de $\mathbb{Q}_{p}$.

Há outras provas como a de S̆afarevič ${ }^{[1]}$ em que a teoria de ramificação aparece de modo mais incisivo do que neste texto.

Uma outra abordagem para a demonstração do teorema de Kronecker-Weber, também utilizando um pouco mais de teoria de números algébricos é a proposta por J. M. Greenberg, ${ }^{[2]}$ o qual fornece uma demonstração bem similar ao nosso texto, porém, utilizando grupos superiores de ramificação.

Atualmente o teorema de Kronecker-Weber é provado como uma conseqüência de teoria de corpos de classe (como por exemplo em [Cohn, 2] ou [GoLdstein, 5]).

Historicamente, Kronecker, em 1853, foi o primeiro a enunciar, em sua publicação "Über die algebraish aufösbaren Gleichungen", o teorema para extensões abelianas de corpos de números. Porém sua demonstração estava incompleta, ele o havia demonstrado para extensões cíclicas e não para extensões abelianas como foi afirmado. Em particular, houve dificuldades com as extensões de grau $2^{n}$.

A primeira tentativa de Heinrich Weber para provar o teorema de Kronecker, foi em 1886 e foi baseada nas principais idéias de Kronecker, mesmo assim, ainda houve um

[1] v. "A New proof of the Kronecker-Weber Theorem", de I. R. S̆afarevič, Tr. Mat. Inst. Steklova, 38, pp. 382-387, 1951..

[2] v. "A Elementary Proof of Kronecker-Weber theorem", American Mathematical Mountly, 81, pp. 601-607, 1974.. 
engano, pois sua demonstração está correta apenas para extensões cíclicas $\mathbb{L} / \mathbb{Q}$ de grau $m$ com a propriedade $\mathbb{L} \cap \mathbb{Q}\left(\zeta_{m}\right)=\mathbb{Q}$. A demonstração de Weber era baseada nos trabalhos de Kronecker sobre extensões cíclicas e no resultado de Kummer sobre períodos gaussianos.

Em 1896/1897, David Hilbert fez uma nova demonstração do teorema de KroneckerWeber baseado em princípios diferentes (teoria de ramificação). Esta prova, está fortemente baseada no teorema do discriminante de Minkowski e sua prova é completa.

O propósito deste trabalho é provar o teorema de Kronecker-Weber sem utilizar a teoria de corpos de classe e tentando utilizar o mínimo possível de teoria algébrica de números.

O texto base para este trabalho, foi o de Neumann, [NEUMANN, 17], que apresenta duas demonstrações do teorema de Kronecker-Weber, uma de acordo com os trabalhos que Kronecker e a outra é uma versão simplificada e corrigida da prova de Weber, porém ambas baseadas em um único teorema, que afirma que extensões cíclicas de grau $\ell^{n}$ sobre $\mathbb{Q}$, onde $\ell$ é um primo, são ciclotômicas.

O primeiro capítulo é apenas um apanhado de teoremas e definições que fornecem um pouco de linguagem e ferramentas para a demonstração do teorema de Kronecker-Weber, como por exemplo, o teorema de Kummer para classes de ideais em corpos ciclotômicos.

O segundo capítulo tem uma caracterização de extensões centrais. Essas extensões são as que Kronecker obtinha, tomando uma extensão cíclica $\mathbb{L} / \mathbb{Q}$ de grau $m$ e juntando raízes $m$-ésimas da unidade. Conseguimos mostrar que um gerador $\mathbb{L}\left(\zeta_{m}\right)$ tem a forma $\mathbb{Q}\left(\zeta_{m}, \sqrt[m]{\Delta(\gamma)}\right)$, onde $\Delta=\sum_{\sigma \in G} r(\sigma) \sigma^{-1}, G$ é o grupo de Galois de $\mathbb{Q}\left(\zeta_{m}\right) / \mathbb{Q}$ e $G \stackrel{r}{\longrightarrow}(\mathbb{Z} / m \mathbb{Z})^{*}$ é o isomorfismo dado por $\sigma\left(\zeta_{m}\right)=\zeta_{m}^{r(\sigma)}$.

O terceiro capítulo expõe fatos sobre corpos ciclotômicos, como o resultado de Kummer sobre Somas de Gauss ou o teorema de Stickelberger. Nesse capítulo, também, é enunciado um teorema de Weber, que claramente é uma generalização do teorema de Kummer sobre Somas de Gauss (ou períodos gaussianos), o qual fornece um gerador explícito para ideais da forma $\Delta(\mathfrak{p})$ onde $\mathfrak{p}$ é um ideal primo que divide $(p)$ em $\mathbb{Q}\left(\zeta_{\ell^{n}}\right)$.

O capítulo 4 está dividido em 2 seções. A primeira seção trata de extensões quadráticas, caso relativamente simples de ser provado e demonstra um teorema chave que mostra que corpos da forma $\mathbb{Q}\left(\zeta_{\ell^{n}}, \sqrt[\ell]{\Delta(\gamma)}\right)$ são ciclotômicos. Este teorema é uma parte importante do teorema de Kronecker-Weber, pois o problema de estudar extensões abelianas de $\mathbb{Q}$ recai sobre o problema de estudar extensões cíclicas de $\mathbb{Q}$. Na segunda seção do capítulo 4 estão as demonstrações de Kronecker e de Weber do famoso teorema.

As finalidades de se retomar as demonstrações originais do teorema de KroneckerWeber, são: 
- As abordagens feitas por Kronecker e Weber são de um ponto de vista "exterior", não utilizando fatos tão aprofundados da teoria de números algébricos;

- As demonstrações originais são, por não usar teorias específicas, mais suscetíveis a serem generalizados para contextos mais amplos. 


\section{Capítulo 1}

\section{Preliminares}

Neste capítulo, abordaremos alguns resultados sobre extensões finitas de corpos, ideais e grupos; tais resultados serão usados nos capítulos seguintes e na demonstração do teorema de Kronecker-Weber.

Chamaremos de corpos de números ou simplesmente corpos, qualquer corpo que seja extensão finita de $\mathbb{Q}$.

\subsection{Extensões de corpos}

Nosso primeiro lema será sobre o compositum de extensões galoisianas que têm grupos de Galois isomorfos. Esse lema será importante quando, no capítulo 4, faremos um compositum de corpos ciclotômicos, mostrando que uma extensão cíclica de $\mathbb{Q}$ de grau primo é ciclotômico.

1.1.1. Lema. Se $\mathbb{L} / \mathbb{K}, \mathbb{M}_{1} / \mathbb{K}, \mathbb{M}_{2} / \mathbb{K}$, são extensões galoisianas tais que $\mathbb{M}_{1} \supset \mathbb{L}, \mathbb{M}_{2} \supset \mathbb{L}$ e

$$
\{1\} \longrightarrow C \longrightarrow G \stackrel{\varphi}{\longrightarrow} \operatorname{Gal}(\mathbb{L} / \mathbb{K}) \longrightarrow\{1\}
$$

é uma seqüência exata curta de grupos onde o grupo $C$ está contido no centro de $G$ e se existirem isomorfismos $\psi_{1}: \operatorname{Gal}\left(\mathbb{M}_{1} / \mathbb{K}\right) \stackrel{\sim}{\longrightarrow} G$ e $\psi_{2}: \operatorname{Gal}\left(\mathbb{M}_{2} / \mathbb{K}\right) \stackrel{\sim}{\longrightarrow} G$, tais que $\varphi \circ \psi_{1}$ e $\varphi \circ \psi_{2}$ coincidem, respectivamente, com os epimorfismos canônicos $\pi_{1}: \operatorname{Gal}\left(\mathbb{M}_{1} / \mathbb{K}\right) \longrightarrow$ $\operatorname{Gal}(\mathbb{L} / \mathbb{K})$ e $\pi_{2}: \operatorname{Gal}\left(\mathbb{M}_{2} / \mathbb{K}\right) \longrightarrow \operatorname{Gal}(\mathbb{L} / \mathbb{K})$, então existe uma extensão galoisiana $\mathbb{E} / \mathbb{K}$ tal que $\mathrm{Gal}(\mathbb{E} / \mathbb{K}) \hookrightarrow C$ e $\mathbb{M}_{1} \cdot \mathbb{M}_{2}=\mathbb{M}_{1} \cdot \mathbb{E}=\mathbb{M}_{2} \cdot \mathbb{E}$; nesta situação, dizemos que $\mathbb{M}_{1}$ e $\mathbb{M}_{2}$ diferem entre si pelo corpo $\mathbb{E}$. 
Demonstração: Sejam $\Sigma_{i}: \operatorname{Gal}\left(\mathbb{M}_{1} \cdot \mathbb{M}_{2} / \mathbb{K}\right) \longrightarrow \operatorname{Gal}\left(\mathbb{M}_{i} / \mathbb{K}\right) \quad(i=1,2)$ os epimorfismos canônicos com as seguintes composições:

$$
\eta_{1}:=\iota_{1} \circ \varepsilon_{1} \quad \eta_{2}:=\iota_{2} \circ \varepsilon_{2} .
$$

A aplicação diferença

$$
\eta_{1}^{-1} \eta_{2}: \operatorname{Gal}\left(\mathbb{M}_{1} \cdot \mathbb{M}_{2} / \mathbb{K}\right) \longrightarrow G
$$

definida por:

$$
g \longmapsto \eta_{1}^{-1} \eta_{2}(g):=\eta_{1}(g)^{-1} \eta_{2}(g),
$$

é um homomorfismo, pois sua imagem está no grupo $C$.

De fato, para todo $g \in \operatorname{Gal}\left(\mathbb{M}_{1} \cdot \mathbb{M}_{2} / \mathbb{K}\right)$, temos

$\varphi\left(\eta_{1}^{-1} \eta_{2}(g)\right)=\varphi\left(\eta_{1}(g)^{-1} \cdot \eta_{2}(g)\right)=\varphi\left(\eta_{1}(g)^{-1}\right) \cdot \varphi\left(\eta_{2}(g)\right)=\left(\varphi \circ \psi_{1} \circ \varepsilon_{1}(g)\right)^{-1} \cdot \varphi \circ \psi_{2} \circ \varepsilon_{2}(g)=$ $\pi_{1}\left(\varepsilon_{1}(g)\right)^{-1} \cdot \pi_{2}\left(\varepsilon_{2}(g)\right)$, e como o diagrama seguinte comuta e $\pi_{1}$ e $\pi_{2}$ são as projeções canônicas temos $\pi_{1}\left(\varepsilon_{1}(g)\right)^{-1} \cdot \pi_{2}\left(\varepsilon_{2}(g)\right)=1$.

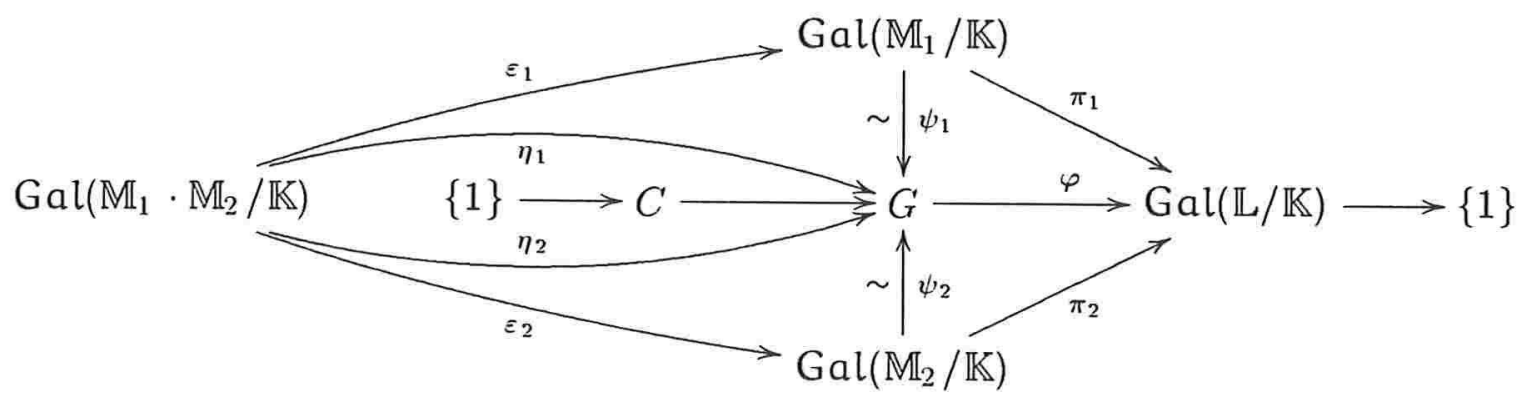
somente se

$$
\left|\begin{array}{l}
\eta_{1}(h)=1_{G} \\
\eta_{2}(h)=1_{G}
\end{array} \Longleftrightarrow\right| \begin{aligned}
& \eta_{1}(h)^{-1} \eta_{2}(h)=1_{G} \\
& \eta_{2}(h)=1_{G}
\end{aligned} \Longleftrightarrow \mid \begin{aligned}
& \eta_{1}(h) \eta_{2}(h)^{-1}=1_{G} \\
& \eta_{1}(h)=1_{G}
\end{aligned} .
$$

Seja $\mathbb{E}$ o corpo fixo por $\operatorname{ker}\left(\eta_{1}^{-1} \eta_{2}\right)$. Como $\psi_{i}$ é um isomorfismo, temos que $M_{i}$ é o corpo fixo por $\operatorname{ker}\left(\eta_{i}\right)$. Além disso, $g \in \operatorname{ker}\left(\eta_{1}\right) \cap \operatorname{ker}\left(\eta_{1}^{-1} \eta_{2}\right) \Longleftrightarrow g \in \operatorname{ker}\left(\eta_{1}\right) \cap \operatorname{ker}\left(\eta_{2}\right) \Longleftrightarrow g=1$, donde $\mathbb{M}_{1} \cdot \mathbb{M}_{2}=\mathbb{M}_{1} \cdot \mathbb{E}$ e, analogamente, $\mathbb{M}_{1} \cdot \mathbb{M}_{2}=\mathbb{E} \cdot \mathbb{M}_{2}$.

Finalmente, $\operatorname{Gal}(\mathbb{E} / \mathbb{K}) \cong \frac{\operatorname{Gal}\left(\mathbb{M}_{1} \cdot \mathbb{M}_{2} / \mathbb{K}\right)}{\operatorname{ker}\left(\eta_{1}^{-1} \eta_{2}\right)} \cong \operatorname{Im}\left(\eta_{1}^{-1} \eta_{2}\right) \subseteq C$. 


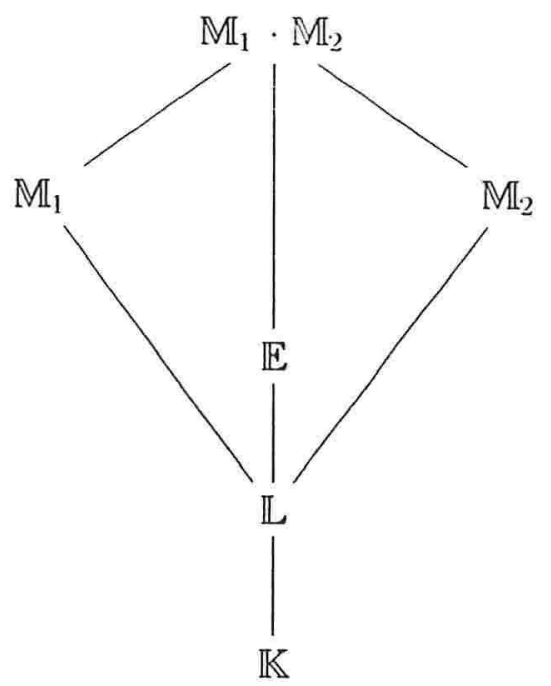

$\{1\}$

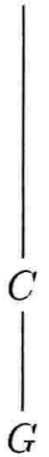

1.1.2. Definição. Se $\mathbb{L} / \mathbb{K}$ é uma extensão galoisiana de corpos de números e $G=$ $\mathrm{Gal}(\mathbb{L} / \mathbb{K})$, definimos a norma de um elemento $\beta \in \mathbb{L}$ como sendo o número

$$
N_{\mathbb{L} / \mathbb{R}^{:}}(\beta):=\prod_{\sigma \in G}(\sigma(\beta))
$$

Vale notar que a norma de qualquer elemento de $\mathbb{L}$ é um elemento do corpo $\mathbb{K}$. Agora estamos em condições de enunciar o teorema "Satz-90" de Hilbert, que caracteriza todos os elementos de norma 1 , em extensões galoisianas cíclicas de $\mathbb{Q}$.

1.1.3. Teorema ("Satz-90" de Hilbert). Sejam $\mathbb{L} / \mathbb{Q}$ uma extensão finita galoisiana cíclica e $\sigma$ um gerador do grupo de Galois de $\mathbb{L} / \mathbb{Q}$. Então para cada elemento $\beta \in \mathbb{L}$, $\beta \neq 0$, tal que $N_{\mathbb{U} / \mathbb{Q}}(\beta)=1$, existe um $\gamma \neq 0, \gamma \in \mathbb{L}$, tal que $\beta=\frac{\sigma(\gamma)}{\gamma}$.

Demonstração: $(\Rightarrow)$ Sejam $m=|\operatorname{Gal}(\mathbb{L} / \mathbb{Q})|$ e $\tau$ um elemento primitivo de $\mathbb{L} / \mathbb{Q}$ tal que o conjunto $\left\{\sigma(\tau), \ldots, \sigma^{m}(\tau)\right\}$ forme uma $\mathbb{Q}$-base de $\mathbb{L}$, cuja existência é garantida pelo teorema da base normal[3]. Tomemos $\beta$ tal que $N_{\mathbb{L} / \mathbb{Q}}(\beta)=1$. Então consideremos o seguinte elemento de $\mathbb{L}$ :

$$
\chi=\sigma(\tau) \beta+\sigma^{2}(\tau) \sigma(\beta) \beta+\cdots+\sigma^{m}(\tau) \sigma^{m-1}(\beta) \sigma^{m-2}(\beta) \ldots \sigma(\beta) \beta
$$

vale notar que \ não é nulo, pois $\left\{\sigma(\tau) \ldots, \sigma^{m}(\tau)\right\}$ é uma $\mathbb{Q}$-base de $\mathbb{L}$, e $\beta \neq 0$. Assim,

$$
\begin{gathered}
\sigma(\chi)=\sigma^{2}(\tau) \sigma(\beta)+\sigma^{3}(\tau) \sigma^{2}(\beta) \sigma(\beta)+\cdots+\sigma(\tau) \sigma^{m}(\beta) \sigma^{m-1}(\beta) \cdots \sigma^{2}(\beta) \sigma(\beta)= \\
=\sigma^{2}(\tau) \sigma(\beta)+\sigma^{3}(\tau) \sigma^{2}(\beta) \sigma(\beta)+\cdots+\sigma(\tau) 1= \\
=\beta^{-1}\left(\sigma^{2}(\tau) \sigma(\beta) \beta+\sigma^{3}(\tau) \sigma^{2}(\beta) \sigma(\beta) \beta+\cdots+\sigma(\tau) \beta\right)=\beta^{-1} \chi,
\end{gathered}
$$

[3] Cf. [ARTiN, 1, pp. 66, 67, teorema 28]. 
ou seja,

$$
\beta=\frac{1}{\sigma(1)}
$$

Se tomarmos $\gamma=\frac{1}{\chi}$, teremos $\beta=\frac{\sigma(\gamma)}{\gamma}$.

Reciprocamente, se $\beta=\frac{\sigma(\gamma)}{\gamma}$ para algum $\gamma \in \mathbb{L}$, então é claro que $N_{\mathbb{L} / \mathbb{Q}}(\beta)=1$, pois $\sigma$ apenas induz uma permutação no grupo $\operatorname{Gal}(\mathbb{L} / \mathbb{Q})$, ou seja, $N_{\mathbb{L} / \mathbb{Q}}(\sigma(\gamma))=N_{\mathbb{L} / \mathbb{Q}}(\gamma)$. 


\subsection{Teoria de números algébricos}

Apresentaremos, nesta seção, alguns fatos sobre teoria de números algébricos. Esses resultados serão utilizados como ferramentas nos capítulos 3 e 4 , quando provaremos alguns resultados sobre corpos ciclotômicos e o próprio teorema de Kronecker-Weber.

Chamaremos de número algébrico qualquer elemento $a \in \mathbb{C}$ tal que a seja raiz de algum polinômio de $\mathbb{Q}\left[\mathrm{Y}^{-}\right]$.

1.2.1. Definição. Sejam $\mathbb{K}$ um corpo de números e $\alpha \in \mathbb{K}$. Dizemos que $\alpha$ é um inteiro de $\mathbb{K}$ ou inteiro algébrico se a for raiz de algum polinômio mônico de $\mathbb{Z}[X]$.

1.2.2. Proposição. Seja $\mathbb{K}$ um corpo de números. São equivalentes:

(a). $\alpha \in \mathbb{K}$ é um inteiro algébrico;

(b). $\mathbb{Z}[\alpha]$ é finitamente gerado.

Demonstração: $(\Rightarrow)$ Seja a uma raiz de algum polinômio mônico com coeficientes inteiros, isto é, existem $a_{1}, \ldots, a_{m} \in \mathbb{Z}$ tais que

$$
\alpha^{m}+a_{1} \alpha^{m-1}+\cdots+a_{m-1} \alpha+a_{m}=0 .
$$

Portanto $\mathbb{Z}[\alpha]=\mathbb{Z} \cdot 1+\mathbb{Z} \cdot \alpha+\cdots+\mathbb{Z} \cdot \alpha^{m-1}$, isto é, $\mathbb{Z}[\alpha]$ é finitamente gerado.

Se $\mathbb{Z}[\alpha]$ é finitamente gerado, então existem $f_{1}, f_{2}, \ldots f_{k} \in \mathbb{Z}[\alpha]$ tais que

$$
\mathbb{Z}[\alpha]=\mathbb{Z} \cdot f_{1}+\cdots+\mathbb{Z} \cdot f_{k} .
$$

Desse modo, se $t$ for o maior expoente de $\alpha$ que aparece em $f_{1}, \ldots, f_{k}$, então temos

$$
\mathbb{Z}[\alpha]=\mathbb{Z} \cdot 1+\cdots+\mathbb{Z} \cdot \alpha^{t} .
$$

Logo, $a^{t+1}=a_{0}+a_{1} \alpha+\cdots+a_{t-1} \alpha^{t-1}+a_{t} a^{t}$ onde $a_{i} \in \mathbb{Z} \quad \forall 1 \leq i \leq t$, isto é, $\alpha$ é inteiro algébrico.

1.2.3. Corolário. Seja $\mathbb{L} / \mathbb{Q}$ uma extensão finita de corpos. Se $\alpha$ e $\beta$ são inteiros algébricos de $\mathbb{L}$, então $\alpha+\beta$ e $\alpha \cdot \beta$ são inteiros algébricos.

Demonstração: Se $\alpha$ e $\beta$ são inteiros algébricos, então $\alpha+\beta \in \mathbb{Z}[\alpha+\beta]$ e $\alpha \cdot \beta \in \mathbb{Z}[\alpha \cdot \beta]$. Notemos que $\mathbb{Z}[\alpha+\beta]$ e $\mathbb{Z}[\alpha \cdot \beta] \subset \mathbb{Z}[\alpha][\beta]=\mathbb{Z}[a . \beta]$. Como $\mathbb{Z}[\alpha]$ é finitamente gerado e $\mathbb{Z}[\beta]$ também, temos que

$$
\begin{aligned}
\mathbb{Z}[\alpha][\beta]= & \mathbb{Z} \cdot 1+\mathbb{Z} \cdot \alpha+\cdots+\mathbb{Z} \cdot \alpha^{t}+ \\
& \mathbb{Z} \cdot \beta+\mathbb{Z} \cdot \alpha \beta+\cdots+\mathbb{Z} \cdot \alpha^{t} \beta+ \\
& \mathbb{Z} \cdot \beta^{2}+\mathbb{Z} \cdot \alpha \beta^{2}+\cdots+\mathbb{Z} \cdot \alpha^{t} \beta^{2}+ \\
& \mathbb{Z} \cdot \beta^{r}+\mathbb{Z} \cdot \alpha \beta^{r}+\cdots+\mathbb{Z} \cdot \alpha^{t} \beta^{r}
\end{aligned}
$$

onde $t$ e $r$ são números inteiros positivos.

Sabemos que anéis de inteiros de corpos de números, em geral, não são domínios de fatoração única. Entretanto se considerarmos os ideais não nulos nesses anéis, então conseguimos recuperar a fatorização unica, fatorando ideais, em vez de números. 
1.2.4. Definição. Quando um domínio integral A, tiver as propriedades:

(a). A é noetheriano;

(b). A é integralmente fechado em seu corpo de frações, ou seja, se $t \in \mathbb{L}=\left\{\frac{\alpha}{\beta}: \alpha, \beta \in\right.$ $A$ e $\beta \neq 0\}$ e té raiz de algum polinômio mônico de $A[\mathrm{Y}]$ então $t \in A$;

(c). todo ideal primo não nulo é maximal;

A será chamado de domínio de Dedekind. ideais.

É fato ${ }^{4]}$ que num domínio de Dedekind, existe fatorização única no conjunto dos

1.2.5. Proposição. Se $\mathbb{L} / \mathbb{Q}$ é uma extensão finita de corpos, então o anel $\mathcal{O}_{\mathbb{L}}$ verifica:

(a). É um domínio com corpo de frações $\mathbb{L}$;

(b). É noetheriano (todo ideal é finitamente gerado);

(c). Se $\alpha \in \mathbb{L}$ é raiz de um polinômio mônico de $\mathcal{O}_{\mathbb{Z}}[\mathrm{Y}]$, então $\alpha \in \mathcal{O}_{\mathbb{L}}$;

(d). Todo ideal primo não nulo de $\mathcal{O}_{\mathbb{L}}$ é maximal.

Demonstração: Isso é o mesmo que dizer que todo anel de inteiros algébricos é um domínio de Dedekind. Portanto a prova é a mesma de [MARCus, 16, p. 56 teorema 14].

1.2.6. Definição. Seja $\mathbb{L} / \mathbb{K}$ uma extensão de corpos de números. Dizemos que $I \subset \mathbb{L}$ é um ideal fracionário de $\mathbb{L}$ se $I$ for um $\mathcal{O}_{\mathbb{L}}$-módulo, tal que existe $c \in \mathcal{O}_{\mathbb{L}} \backslash\{0\}$, verificando $c I \subset \mathcal{O}_{\mathbb{L}}$.

1.2.7. Definição. Seja $\mathbb{L} / \mathbb{K}$ uma extensão de corpos de números. Um ideal fracionário do tipo $y \mathcal{O}_{\mathbb{L}}$, com $y \in \mathbb{L}$, é chamado de ideal fracionário principal.

1.2.8. Definição. Seja $\mathbb{L} / \mathbb{K}$ uma extensão de corpos de números. Um ideal a em $\mathcal{O}_{\mathbb{L}}$ é invertível se existir um ideal $\mathfrak{b}$ em $\mathcal{O}_{\mathbb{L}}$ tal que $\mathfrak{a} \cdot \mathfrak{b}=\mathcal{O}_{\mathbb{L}}$.

1.2.9. Definição. Seja $\mathbb{L} / \mathbb{K}$ uma extensão de corpos de números e seja $I$ um ideal fracionário de $\mathbb{L}$. Definimos o inverso de $I$ como o conjunto $I^{-1}=\left\{x \in \mathbb{L}: x I \subset \mathcal{O}_{\mathbb{L}}\right\}$.

1.2.10. Proposição. $I^{-1}$ é ideal fracionário de $\mathbb{L}$ e caso $I$ seja ideal invertível, então $I^{-1}$ será um ideal em $\mathcal{O}_{\mathbb{L}}$.

Demonstração: v. [Goldstein, 7, p. 14]

[4] Cf. [Marcus, 16, p. 59 teorema 16]. 


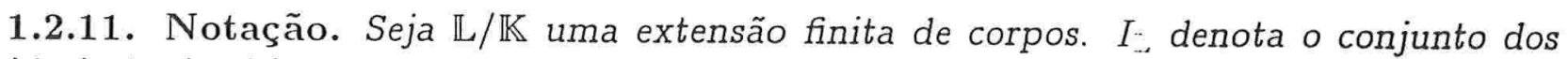
ideais fracionários de $\mathbb{L}$.

Sabemos que, num domínio de Dedekind $(A)$ que não é corpo, todo ideal se fatora como produto de ideais primos de forma única exceto pela ordem. Mais ainda, qualquer ideal não nulo invetível se fatora como produto de ideais primos invertíveis. [5]

Do fato de todo ideal não nulo ser invertível, temos que o conjunto dos ideais não nulos em A forma um grupo abeliano. Se tomarmos um ideal não nulo, a $\in I_{\mathbb{L}}$, então $\mathfrak{a}=\mathfrak{b}(b)^{-1}$, onde $\mathfrak{b}$ é um ideal em $A$ e $b \in A$ então podemos expressar $\mathfrak{b}$ e $(b)$ como produto de ideais primos. Claramente, se $(b)=\prod \mathfrak{p}_{j}$ então $(b)^{-1}=\prod \mathfrak{p}_{j}^{-1}$ e agrupando todos os ideais primos iguais, temos

$$
\mathfrak{a}=\prod_{\mathfrak{p}} \mathfrak{p}^{v_{p}} \quad v_{\mathfrak{p}}(\mathfrak{a}) \in \mathbb{Z}
$$

Este produto pode ser estendido formalmente a todos os ideais primos. Com efeito, a é um produto finito de primos, portanto $v_{\mathfrak{p}}(\mathfrak{a})$ é nulo para quase todos os ideais primos $\mathfrak{p}$, exceto para um conjunto finito (os divisores de $\mathfrak{a}$ ).

1.2.12. Definição. Em um domínio de Dedekind $A$, dizemos que um ideal a $\subset A$ divide um ideal $\mathfrak{b} \subset A$ se existir um ideal $\mathfrak{c} \subset A$ tal que $\mathfrak{a} \cdot \mathfrak{c}=\mathfrak{b}$.

Vale observar que, dizer um ideal $\mathfrak{a}$ divide $\mathfrak{b}$ é o mesmo que dizer $\mathfrak{a}$ está contido em $\mathfrak{b}$.

1.2.13. Proposição. Sejam a e b ideais não nulos de um domínio de Dedekind A. Então são equivalentes:

(a). $\mathfrak{a}+\mathfrak{b}=\mathfrak{A}$;

(b). $\mathfrak{a} \cap \mathfrak{b}=\mathfrak{a} \cdot \mathfrak{b}$;

(c). $v_{\mathfrak{p}}(\mathfrak{a}) \cdot v_{\mathfrak{p}}(\mathfrak{b})=0 \quad \forall \mathfrak{p}$. Neste caso, dizemos que $\mathfrak{a}$ e $\mathfrak{b}$ são primos entre si.

Demonstração: v. [FRÖLICH, 4, p. 43, teorema 1.15.g]

1.2.14. Proposição. Se $\mathfrak{p}$ for um ideal primo não nulo de um domínio de Dedekind $A e$ seja $r \in \mathbb{N}$, então temos o isomorfismo de grupos aditivos:

$$
A / \mathfrak{p} \cong \mathfrak{p}^{r} / \mathfrak{p}^{r+1}
$$

Demonstração: v. [FRÖLICH, 4, p. 43, teorema 1.16]

[5] Cf. Frölich, 4, pp. 39, 40 e 41. 
1.2.15. Teorema. Seja $\mathbb{L} / \mathbb{Q}$ uma extensão galoisiana finita de corpos e $\mathcal{O}_{\mathbb{L}}$ o anel de inteiros de $\mathbb{L}$. Então, se $I$. $J \in I_{\text {- temos }}$

(a). I é um ideal de $\mathcal{O}_{\mathrm{L}}$ se e somente se, $\iota_{\mathrm{p}}(I) \geq 0$, para todo ideal primo p de $\mathcal{O}_{\mathbb{E}}$;

(b). $\left\{\begin{array}{l}v_{\mathrm{p}}(I+J)=\min \left\{v_{\mathrm{p}}(I) \cdot v_{\mathrm{p}}(J)\right\} \\ v_{\mathrm{p}}(I \cdot J)=v_{\mathrm{p}}(I)+v_{\mathrm{p}}(J) \\ v_{\mathrm{p}}(I \cap J)=\max \left\{v_{\mathrm{p}}(I), v_{\mathrm{p}}(J)\right\} .\end{array}\right.$

Demonstração: (a). v. [MARCus, 16, pp. 56, 59, 60, teoremas 14 e 16].

(b). v. [Goldstein, 5, p. 27].

1.2.16. Definição. Seja $\mathbb{L} / \mathbb{K}$ uma extensão de corpos de números e sejam $\mathcal{O}_{\mathbb{L}}$ o anel de inteiros de $\mathbb{L}$ e $\mathfrak{p} \subset \mathbb{K}$ um ideal primo. Definimos o índice de ramificação de $\mathfrak{P}_{i}$ como o $e_{i}$ da decomposição de $\mathfrak{p} \mathcal{O}_{\mathbb{L}}$ em ideais primos $\mathfrak{p} \mathcal{O}_{\mathbb{L}}=\mathfrak{P}_{1}^{e_{1}} \cdots \mathfrak{P}_{n}^{e_{n}}$, em $\mathcal{O}_{\mathbb{L}}$. Se, para algum $i, \epsilon_{i}>1$, dizemos que $\mathfrak{P}_{i}$ é ramificado em $\mathbb{L} / \mathbb{K}$ ou que $\mathfrak{p}$ ramifica em $\mathbb{L}$, caso $\epsilon_{i}=1$ para todo $i$, dizemos que $\mathfrak{P}_{i}$ é não-ramificado em $\mathbb{L}$ ou que $\mathfrak{p}$ não ramifica em $\mathbb{L}$ e a cada primo $\mathfrak{P}_{i}$ em que $\epsilon_{i} \geq 1$ dizemos que $\mathfrak{P}_{i}$ está sobre $\mathfrak{p}$ ou que $\mathfrak{p}$ está abaixo de $\mathfrak{P}_{i}$.

1.2.17. Definição. Sejam $\mathbb{L} / \mathbb{Q}$ uma extensão de corpos de números, $\mathcal{O}_{\mathbb{L}}$ o anel de inteiros de $\mathbb{L}$, e $\mathfrak{P}_{i}$ um ideal primo em $\mathcal{O}_{\mathbb{L}}$, chamaremos de corpo de restos de $\mathfrak{P}_{i}$ o corpo $\mathcal{O}_{\mathbb{L}} / \mathfrak{P}_{i}$.

Se $\mathbb{L} / \mathbb{Q}$ é uma extensão finita e $(p) \subset \mathbb{Z}$ um ideal primo, tomemos $\mathfrak{P}_{1} \cdots \mathfrak{P}_{g}$ a decomposição de $p \mathcal{O}_{\mathbb{L}}$ em $\mathcal{O}_{\mathbb{L}}$, então o corpo $\mathcal{O}_{\Xi} / \mathfrak{P}_{i}$ será uma extensão finita de $\mathbb{Z} / p \mathbb{Z}$, e portanto $\mathcal{O}_{\mathbb{L}} / \mathfrak{P}_{i}$ será finito.

1.2.18. Definição. Sejam $\mathbb{L} / \mathbb{K}$ uma extensão de corpos de números, consideremos um ideal primo $\mathfrak{p} \subset \mathcal{O}_{\mathbb{L}}, \mathcal{O}_{\mathbb{L}}$ o anel de inteiros de $\mathbb{L}$ e $\mathfrak{P}_{1}^{\epsilon_{1}} \cdots \mathfrak{P}_{n}^{e_{n}}$ a decomposição de $\mathfrak{p} \mathcal{O}_{\mathbb{L}}$ em ideais primos de $\mathcal{O}_{\mathbb{L}}$. Chamaremos de grau de $\mathfrak{P}_{i}$ o número $f\left(\mathfrak{P}_{i} / \mathfrak{p}\right):=\left[\mathcal{O}_{\mathbb{L}} / \mathfrak{P}_{i}: \mathcal{O}_{\mathbb{E}} / \mathfrak{p}\right]$, que denotaremos simplesmente por $f_{i}$ quando não houver perigo de confusão.

1.2.19. Teorema. Sejam $\mathbb{K} / \mathbb{Q}$ uma extensão de grau $n, \mathcal{O}_{\mathbb{R}}$ seu anel de inteiros, $p$ um primo racional, $\mathfrak{p}_{1}, \mathfrak{p}_{2}, \ldots, \mathfrak{p}_{g}$ primos em $\mathcal{O}_{\mathbb{k}}$ que estão sobre $(p)$. Sejam $\epsilon_{i}$ e $f_{i}$ o índice de ramificação e o grau de $\mathfrak{p}_{i}$. Então

$$
\sum_{i=1}^{g} e_{i} \cdot f_{i}=n
$$

Demonstração: v. [ENDLER, 3, p. 104, teorema 12.3] 
1.2.20. Definição. Seja $\mathrm{X}$ um conjunto. Seja $G$ um subgrupo do grupo das aplicações bijetoras de $\mathrm{X}$ em $\mathrm{X}$. Dizemos que $G$ age transitivamente sobre $\mathrm{X}$ se dados dois elementos $a . b \in \mathrm{X}$, existe um elemento $\sigma \in G_{r}^{\prime}$ tal que $\sigma(a)=b$.

1.2.21. Teorema. Sejam $\mathbb{L} / \mathbb{K}$ uma extensão galoisiana de grau n e p um ideal primo em $\mathbb{K}$. Então o grupo de Galois de $\mathbb{L} / \mathbb{K}$ age transitivamente sobre os primos que estão sobre p.

Demonstração: v. [MARCUS, 16, pp. 70, 71, teorema 23] ou [SERRE, 19, p. 20, proposição 19]

1.2.22. Corolário. Seja $\mathbb{K} / \mathbb{Q}$ uma extensão galoisiana de grau n e sejam $p$ um primo racional, $\mathfrak{p}_{1}, \mathfrak{p}_{2}, \ldots, \mathfrak{p}_{g}$ ideais primos em $\mathbb{K}$ que estão sobre $(p)$. Sejam $\epsilon_{i}$ e $f_{i}$ o índice de ramificação e o grau de $\mathfrak{p}_{i}$. Então

$$
\epsilon_{1}=\epsilon_{2}=\cdots=\epsilon_{g} \quad \text { e } \quad f_{1}=f_{2}=\cdots=f_{g} .
$$

Demonstração: Basta notar que $\sum_{i=1}^{g} e_{i} \cdot f_{i}=n$ e que $\operatorname{Gal}(\mathbb{K} / \mathbb{Q})$ age transitivamente sobre os primos que estão sobre $(p)$. Assim, se $(p)=\mathfrak{p}_{1}^{e_{1}} \mathfrak{p}_{2}^{e_{2}} \ldots \mathfrak{p}_{g}^{e_{g}}$ então $\left(\sigma_{1,2}(p)\right)=$ $(p)=\mathfrak{p}_{2}^{e_{1}} \mathfrak{p}_{1}^{e_{2}} \ldots \mathfrak{p}_{g}^{e_{g}}$, onde $\sigma_{1,2}$ leva o ideal primo $\mathfrak{p}_{1}$ no ideal primo $\mathfrak{p}_{2}$. Como a decomposição de $(p)$ em fatores primos é única, temos que $\epsilon_{1}=\epsilon_{2}$. Analogamente provamos que $\epsilon_{i}=\epsilon_{1} \quad \forall i \leq g$. A igualdade de $f_{i}=f_{j}$, é obtida através do isomorfismo $\sigma_{1,2}: \mathcal{O}_{\mathbb{K}} \longrightarrow \mathcal{O}_{\mathbb{K}}, \quad \sigma_{1,2} \in \operatorname{Gal}(\mathbb{K} / \mathbb{Q})$, pois

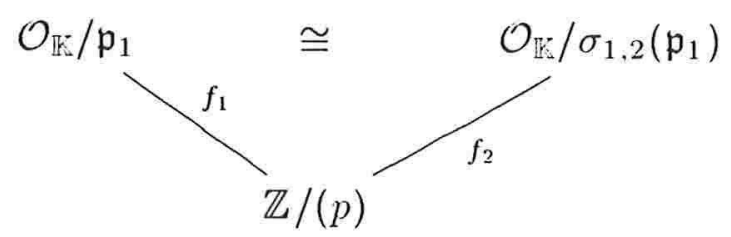

e portanto $f_{1}=f_{2}$. Analogamente concluímos que $f_{i}=f_{1}$.

1.2.23. Corolário. Sejam $\mathbb{K} / \mathbb{Q}$ uma extensão galoisiana de grau $n$, $\mathcal{O}_{\mathbb{K}}$ o anel de inteiros de $\mathbb{K}, p$ um primo racional e $\mathfrak{p}_{1}, \ldots, \mathfrak{p}_{g}$ os ideais primos distintos de $\mathcal{O}_{\mathbb{K}}$ sobre $(p)$. Sejam e e $f$ o índice de ramificação e o grau do ideal primo $\mathfrak{p}_{i}$, respectivamente, então $e \cdot f \cdot g=n$.

Demonstração: Decorrência direta de $\sum_{i=1}^{g} \epsilon_{i} \cdot f_{i}=n$, pois todos os índices de ramificação são iguais e os graus também. 
1.2.24. Definição. Seja At um domínio de integridade. Uma valorização discreta no corpo de frações $\mathbb{L}$ de $A$ é uma aplicação $v: \mathbb{L} \longrightarrow \mathbb{Z} \cup\{\infty\}$, tal que $v(x \cdot y)=v(x)+v(y)$, $v(x+y) \geq \min \{v(x), v(y)\}$ e $v(x)=\infty \Longleftrightarrow x=0$.

1.2.25. Definição. Seja A um domínio de integridade e v uma valorização discreta no corpo de frações, $\mathbb{L}$ de $A$, definimos:

(a). $O$ anel de valorização de $v$ como o conjunto $A_{v}=\{x \in \mathbb{L}: v(x) \geq 0\}$;

(b). O ideal de valorização de $v$ como o conjunto $P_{v}=\{x \in \mathbb{L}: v(x)>0\}$.

1.2.26. Observação. Seja $A_{v}$ um anel de valorização e seja $x \in A_{v}, x \neq 0$, se $x^{-1} \in A_{v}$ então $v(x) \geq 0$ e $v\left(x^{-1}\right) \geq 0$, o que implica que $v(x)=0$. Portanto $x$ é invertível se $e$ somente se $v(x)=0$ o que equivale a $x \in A_{v} \backslash P_{v}$. Conseqüentemente, $P_{v}$ é o único ideal maximal de $A_{v}$.

1.2.27. Definição. Seja $\mathcal{O}_{\mathbb{L} v}$ um anel de valorização, $v$ a valorização de $\mathbb{L}$ (o corpo de frações de $\mathcal{O}_{\mathbb{L} v}$ ) e $P_{v}$ o ideal de valorização tal que $\mathcal{O}_{\mathbb{L} v} / P_{v}$ seja finita. Chamaremos qualquer elemento $\pi \in P_{v}$ tal que $\pi \mathcal{O}_{\mathbb{L} v}=P_{v}$ de uniformizante local, chamaremos de unidade de $\mathcal{O}_{\mathbb{L} v}$ todo elemento $x \in \mathcal{O}_{\mathbb{L} v}$ em que $v(x)=0$.

Para se obter um uniformizante local, basta tomar qualquer elemento de $P_{v}-P_{v}^{2}$.

1.2.28. Observação. Dois uniformizantes locais de um anel de valorização $\mathcal{O}_{\mathbb{L} v}$, diferem multiplicativamente por uma unidade. De fato, escolheremos um uniformizante local $\pi$ qualquer, se $\pi^{\prime}$ for um outro uniformizante local, temos $v\left(\pi \cdot \pi^{\prime-1}\right) \in \mathcal{O}_{\mathbb{L} v} \backslash P_{v}$, portanto $\pi \cdot \pi^{\prime-1}$ é uma unidade de $\mathcal{O}_{\mathbb{L} v}$, ou seja, quaisquer dois uniformizantes locais diferem multiplicativamente por uma unidade de $\mathcal{O}_{\mathbb{L} v}$.

1.2.29. Definição. Sejam $\mathbb{L} / \mathbb{K}$ uma extensão galoisiana de corpos de números, $\mathfrak{P}$ um ideal primo em $\mathbb{L}$ que contém $\mathfrak{p} \mathcal{O}_{\mathbb{L}}$, onde $\mathfrak{p}$ é um ideal deal primo em $\mathcal{O}_{\mathbb{K}}$. O subgrupo de $\operatorname{Gal}(\mathbb{L} / \mathbb{K})$ que fixa $\mathfrak{P}$ é chamado grupo de decomposição de $\mathfrak{P}$ sobre $\mathfrak{p}$ em $\mathbb{L} / \mathbb{Q}$. Denotaremos tal grupo por $D_{\mathfrak{P} / \mathfrak{p}}(\mathbb{L} / \mathbb{Q})$ ou simplesmente, $D_{\mathfrak{P} / \mathfrak{p}}$, quando não houver ambigüidade.

1.2.30. Proposição. Seja $\mathbb{L} / \mathbb{K}$ uma extensão finita galoisiana de corpos de números. Seja $\mathfrak{p} \subset \mathcal{O}_{\mathbb{E}}$ um ideal primo. Se $\mathfrak{P}^{\prime}$ e $\mathfrak{P}$ forem dois ideais primos sobre $\mathfrak{p}$, então $D_{\mathfrak{P}^{\prime}}$ é conjugado de $D_{\mathfrak{P}}$.

Demonstração: v. [ENDLER, 3, p. 175, teorema 20.4] 
1.2.31. Proposição. Seja $\mathbb{L} / \mathbb{K}$ uma extensão finita galoisiana de corpos de números. Seja p um ideal primo em $\mathcal{O}_{\mathbb{K}}, \mathfrak{P}$ um ideal primos acima de $\mathfrak{p}$ com índice de ramificação $\epsilon$, grau $f$ e $D_{\mathfrak{P}}$ o grupo de decomposição de $\mathfrak{P}$. Então o índice de $D_{\mathfrak{P}}$ em $\mathrm{Gal}(\mathbb{L} / \mathbb{K})$ é igual a $g$ e a ordem de $D_{\mathfrak{P}}$ é igual a $\epsilon \cdot f$.

Demonstração: v. [ENDLER, 3, p. 176, teorema 20.7]

1.2.32. Corolário. Sob as hipóteses da proposição anterior, $g=1$ se e somente se $D_{\mathfrak{P}}=\operatorname{Gal}(\mathbb{L} / \mathbb{K})$ e $g=[\mathbb{L}: \mathbb{K}]$ se e somente se $D_{\mathfrak{P}}$ for o grupo trivial.

Demonstração: $g=1 \Longleftrightarrow[\mathbb{L} / \mathbb{K}]=1 \Longleftrightarrow\left[\operatorname{Gal}(\mathbb{L} / \mathbb{K}): D_{\mathfrak{P}}\right]=1 \Longleftrightarrow D_{\mathfrak{P}}=$ $\operatorname{Gal}(\mathbb{L} / \mathbb{K})$ e $g=[\mathbb{L}: \mathbb{K}] \Longleftrightarrow e(\mathfrak{P} /(p))=1$ e $f(\mathfrak{P} /(p))=1$ pois $\epsilon \cdot f \cdot g=1 \Longleftrightarrow D_{\mathfrak{P}}$ é trivial.

1.2.33. Definição. Sejam $\mathbb{L} / \mathbb{K}$ é uma extensão finita galoisiana, p um ideal primo em $\mathbb{K}$ e $\mathfrak{P}$ um ideal primo acima de $\mathfrak{p}$, então o grupo $D_{\mathfrak{P}}$ corresponde a um corpo chamado corpo de decomposição de $\mathfrak{p}$, ou simplesmente corpo de decomposição, quando não houver ambigüidade e denotaremos esse corpo por $\mathbb{L}^{D_{\Downarrow 3}}$.

1.2.34. Observação. $\mathbb{L} / \mathbb{L}^{D_{\mathfrak{\beta}}}$ é uma extensão galoisiana finita cujo grupo de Galois é $D_{\mathfrak{P}}$.

Essa proposição quer dizer que o corpo de decomposição pode ser caracterizado como o menor corpo $\mathbb{K}^{\prime}$ entre $\mathbb{K}$ e $\mathbb{L}$ tal que $\mathfrak{P}$ seja o único ideal primo de $\mathcal{O}_{\mathbb{L}}$ (anel de inteiros de $\mathbb{L}$ ) acima de $\mathfrak{P} \cap \mathcal{O}_{\mathbb{K}^{\prime}}$ onde $\mathcal{O}_{\mathbb{K}^{\prime}}^{\prime}=\mathcal{O}_{\mathbb{L}} \cap \mathbb{K}^{\prime}$.

1.2.35. Definição. Sejam $\mathbb{L} / \mathbb{K}$ extensão finita galoisiana, $\mathfrak{p} \subset \mathbb{K}$ um ideal primo $\mathfrak{P}$ um ideal primo em $\mathbb{L}$ sobre $\mathfrak{p} \mathcal{O}_{\mathbb{L}}, D_{\mathfrak{P} / \mathfrak{p}}$ o grupo de decomposição de $\mathfrak{P}$ sobre $\mathfrak{p}$, onde $\mathcal{O}_{\mathbb{L}}$ é o anel de inteiros de $\mathbb{L}$ sobre $\mathbb{K}$. O subgrupo de $\operatorname{Gal}(\mathbb{L} / \mathbb{K})$ definido por

$$
\left\{\sigma \in D_{\mathfrak{P} / \mathfrak{p}} \mid \sigma(\alpha)-\alpha \in \mathfrak{P} \forall \alpha \in \mathcal{O}_{\mathbb{L}}\right\}
$$

será chamado o grupo de inércia de $\mathfrak{P}$ sobre $\mathbb{K}$, que será denotado por $T_{\mathfrak{P} / \mathfrak{p}}$.

Denotaremos, respectivamente, por $D_{\mathfrak{P}}$ e $T_{\mathfrak{p}}$ ou simplesmente por $D$ e $T$ os grupos de decomposição e inércia, quando $\mathfrak{p}$ for fixo ou não houver perigo de ambigüidade.

Se $\mathbb{E}$ é um corpo entre $\mathbb{K}$ e $\mathbb{L}$, seja $\mathcal{O}_{\mathbb{E}}=\mathbb{E} \cap \mathcal{O}_{\mathbb{L}} \circ$ fecho integral de $\mathcal{O}_{\mathbb{K}}$ em $\mathbb{E}$, $\mathfrak{P}_{\mathbb{E}}=\mathfrak{P} \cap \mathcal{O}_{\mathbb{E}}$, e denotaremos por $\widetilde{E}$ o corpo de resíduos $\mathcal{O}_{\Xi} / \mathfrak{P}_{\mathbb{E}}$. Em particular, isso se aplica a $\mathbb{K}$ e $\mathbb{L}$, definindo os corpos $\underset{\widetilde{K}}{\mathbb{K}}$ e $\widetilde{\mathbb{L}}$. Se $s \in D$, s define, por passagem ao quociente, um automorfismo $\widetilde{s}$ de $\widetilde{\mathbb{L}}$, que fixa $\widetilde{\mathbb{K}}$. Portanto, obtemos um homomorfismo

$$
\varepsilon: D \longrightarrow \operatorname{Gal}(\tilde{\mathbb{L}} / \tilde{\mathbb{K}})
$$

cujo kernel será chamado $\widetilde{T}$. 
1.2.36. Proposição. O homomorfismo

$$
\Xi: D \longrightarrow \operatorname{Gal}(\tilde{\mathbb{L}} / \widetilde{\mathbb{K}})
$$

define um isomorfismo de $D / T$ sobre $\operatorname{Gal}(\widetilde{\mathbb{L}} / \widetilde{\mathbb{K}})$.

Demonstração: v. [SERRE, 19, p. 31, teorema 20]

1.2.37. Corolário. Seja $\mathbb{L} / \mathbb{K}$ uma extensão galoisiana de corpos de números. A seguinte seqüencia de grupos é exata:

$$
\{1\} \longrightarrow T \longrightarrow D \longrightarrow \operatorname{Gal}(\tilde{\mathbb{L}} / \widetilde{\mathbb{K}}) \longrightarrow\{1\}
$$

Demonstração: Decorrência direta da proposição anterior.

1.2.38. Teorema. Sejam $\mathbb{L} / \mathbb{Q}$ uma extensão galoisiana finita, $p \in \mathbb{Z}$ um primo que não divida $[\mathbb{L}: \mathbb{Q}]$ e $\mathfrak{P}$ um ideal primo de $\mathcal{O}_{\mathbb{Z}}$ acima de $(p)$. Então $\in(\mathfrak{P} / \mathbb{Q})$ divide $p^{f}-1$ onde $f=f(\mathfrak{P} / \mathbb{Q})$.

Demonstração: v. [IYANAGA, 11, p. 155, corolário do teorema 6.8]

1.2.39. Proposição. $\widetilde{\mathbb{L}} / \widetilde{\mathbb{K}}$ tem grupo de Galois $D / T$ e valem:

$$
\left[\mathbb{L}: \mathbb{L}^{T}\right]=e, \quad\left[\mathbb{L}^{T}: \mathbb{L}^{D}\right]=f, \quad\left[\mathbb{L}^{D}: \mathbb{K}\right]=g .
$$

Demonstração: v. [SERRE, 19, p. 22, corolário 22]

Apresentamos aqui, as torres de corpos com seus respectivos graus e o que ocorre com $(p)$ em cada uma das extensões de $\mathbb{K}$

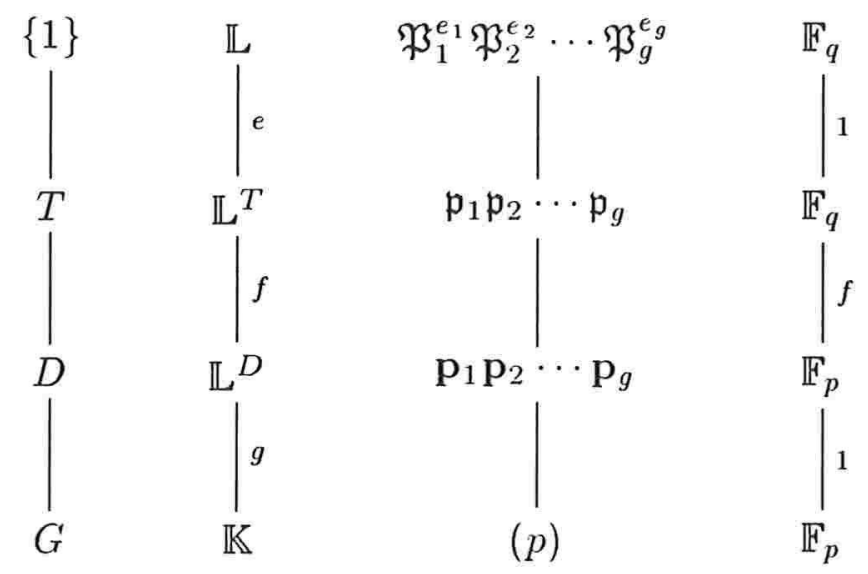

1.2.40. Definição. Sejam $\mathbb{L} / \mathbb{K}$ uma extensão galoisiana de corpos de números, $A$ e $B$ ideais em $\mathbb{L}$ e $\mathbb{K}$, respectivamente. Definimos o codiferente de $A$ relativo a $B$ como $A^{*}:=\left\{\alpha \in \mathbb{L}: \operatorname{Tr}_{\mathbb{L} / \mathbb{K}}(\alpha A) \subset B\right\}$ e denotaremos por $\mathfrak{D}_{\mathbb{L} / \mathbb{K}}(A)$. Quando $A=\mathcal{O}_{\mathbb{L}}$ e $B=\mathcal{O}_{\mathbb{K}}$, dizemos que $A^{*}$ é o codiferente da extensão $\mathbb{L} / \mathbb{K}$ e denotaremos por $\mathfrak{D}_{\mathbb{L} / \mathbb{K}}$. 
1.2.41. Definição. Sejam $\mathbb{L} / \mathbb{K}$ uma extensão galoisiana de corpos de números, $f$ um ideal de $\mathbb{L}, B$ um ideal de $\mathbb{K}$ e $A^{*}$ o codiferente de $A$ relativo a $B$. Definimos o diferente de $A$ como o ideal inverso de $A^{*}$. Quando $A=\mathcal{O}$ e e $B=\mathcal{O}$, dizemos que $A^{*-1}$ é o diferente de $\mathbb{L} / \mathbb{K}$.

1.2.42. Teorema (Divisor). Seja $\mathbb{L} / \mathbb{K}$ uma extensão galoisiana finita. Então o diferente $\mathfrak{D}_{\mathbb{L} / \mathbb{E}}$ divide o diferente de $\mathfrak{D}_{\mathbb{L} / \mathbb{E}}\left(a \mathcal{O}_{\mathbb{Z}}\right)$ para qualquer a é um inteiro algébrico de $\mathbb{L}$ sobre $\mathbb{K}$.

Demonstração: Sejam $m$ o grau de $\mathbb{L} / \mathbb{K}, \theta$ um elemento primitivo de $\mathbb{L}$ sobre $\mathbb{K}$. Denotaremos $\theta^{(n)}$, a $n$-ésima imersão de $\theta$ em $\overline{\mathbb{K}}$ e seja $f(x)=\prod_{\nu=0}^{n-1}\left(x-\theta^{(\nu)}\right)$ o polinômio característico de $\theta$, tal que $D(\theta)=f^{\prime}(\theta)$ seja o diferente de $\theta$. Seja $g(x)$ qualquer polinômio sobre $\mathbb{K}$ de grau menor ou igual a $m$.

Como $\mathbb{L} / \mathbb{K}$ é separável e $\left|\left(\theta^{(\nu)}\right)^{i}\right|_{i, \nu=0 \ldots \ldots m-1} \neq 0$, existe somente um polinômio (com coeficientes em alguma extensão de $\mathbb{K}$ ) de grau menor ou igual a $m$, que assume, para os argumentos $\theta^{(\nu)}$, o valor $g\left(\theta^{(\nu)}\right)$, e que é o próprio polinômio $g(x)$.

Esta única determinação de $g(x)$ por seus valores $g\left(\theta^{(\nu)}\right)$ é expressa computacionalmente pela fórmula de interpolação de Lagrange

$$
g(x)=\sum_{i=0}^{m-1} \frac{g\left(\theta^{(\nu)}\right)}{f^{\prime}\left(\theta^{(\nu)}\right)} \frac{f(x)}{x-\theta^{(\nu)}},
$$

na qual o lado direito é polinômio (com coeficientes em algum fecho algébrico de $\mathbb{L}$ sobre $\mathbb{K})$, de grau menor do que $m$ que assume os valores $g\left(\theta^{(\nu)}\right)$ para $\theta^{(\nu)}$. Essa fórmula pode ser reescrita como

$$
g(x)=T r_{\mathbb{L} / \mathbb{E}}\left(\frac{g(\theta)}{D(\theta)} \frac{f(x)}{x-\theta}\right) .
$$

Se concordarmos que o traço de um polinômio sobre $\mathbb{K}$ é para ser entendido coeficiente a coeficiente, isto é,

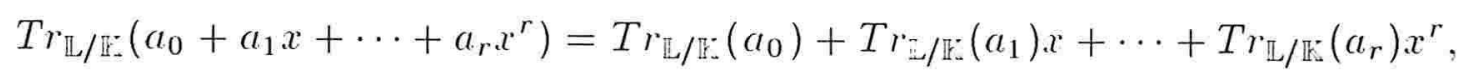

então mostraremos que $\mathfrak{D}$ divide $D(\theta)$.

Para isso, precisamos mostrar que todo múltiplo de $\frac{1}{\mathfrak{D}}$ em $\mathbb{L}$ é um múltiplo de $\frac{1}{D(\theta)}$, portanto que o elemento $g \in \mathbb{L}$ definido por $a=\frac{g}{D(\theta)}$ é inteiro. Se colocarmos $g=g(\theta)$, com o polinômio $g(x)$ sobre $\mathbb{K}$ de grau menor que $m$, então de acordo com a fórmula de interpolação de Lagrange,

$$
T r_{\mathbb{L} / \mathbb{E}}\left(a \frac{f(x)}{x-\theta}\right)=g(x) .
$$


Desde que o polinômio $\frac{f(x)}{x-\theta}=\prod_{\nu=1}^{n-1}\left(x-\theta^{(\nu)}\right)$ tem coeficientes interos, os coeficientes de $a \frac{f(x)}{x-\theta}$ são múltiplos de $\frac{1}{\mathfrak{D}}$. Portanto, pela definição do diferente, os traços desses coeficientes são inteiros. Portanto $g(x)$ tem coeficientes inteiros e como $g=g(\theta)$ é um inteiro de $\mathbb{L}$.

Como os diferentes dos elementos não primitivos são nulos, temos que o diferente $\mathfrak{D}$ é um divisor comum de todos os diferentes dos elementos inteiros de $\mathbb{L}$.

1.2.43. Definição. Sejam $\mathbb{L} / \mathbb{Q}$ uma extensão de corpos, galoisiana, finita e $\left(\theta_{1}, \ldots, \theta_{n}\right)$ uma $n$-upla de números algébricos de $\mathbb{L}$. Definimos o discriminante de $\left(\theta_{1}, \ldots, \theta_{n}\right)$, $\mathbb{L} / \mathbb{Q}$ como

$$
\mathfrak{d}_{\mathbb{L} / \mathbb{Q}}\left(\theta_{1}, \ldots \theta_{n}\right)=\operatorname{det}\left(T r_{\mathbb{L} / \mathfrak{G}}\left(\theta_{i} \theta_{j}\right)\right)
$$

1.2.44. Teorema. Seja $\mathbb{L} / \mathbb{Q}$ uma extensão galoisiana finita de corpos de grau $n$ e sejam $\left\{\theta_{1}, \ldots \theta_{n}\right\},\left\{\omega_{1} \ldots \ldots \omega_{n}\right\}$ duas bases de inteiros algébricos de $\mathbb{L} / \mathbb{Q}$. Então

$$
\mathfrak{d}_{\mathbb{L} / \mathbb{Q}}\left(\theta_{1}, \ldots, \theta_{n}\right)=\mathfrak{d}_{\mathbb{L} / \mathbb{Q}}\left(\omega_{1}, \ldots \omega_{n}\right) .
$$

Demonstração: v. [Rosen, M \& IRELAND. K, 18, pp. 173, 174, 175, 176, proposições $12.1 .2,12.2 .2]$

1.2.45. Observação. O discriminante é invariante em relação à base de inteiros algébricos. Portanto podemos definir o discriminante de $\mathbb{L} / \mathbb{Q}$, como o discriminante de qualquer base de inteiros algébricos de $\mathbb{L}$.

1.2.46. Teorema. Seja $\mathbb{K} / \mathbb{Q}$ uma extensão tal que $[\mathbb{K}: \mathbb{Q}]>1$, então $\mathbb{K} / \mathbb{Q}$ tem discriminante maior que 1.

Demonstração: v. [MARCus, 16, p. 137 corolário 13]

1.2.47. Teorema. Seja $\mathbb{K} / \mathbb{Q}$ uma extensão de corpos de números de grau n. Então um primo $p$ de $\mathbb{Z}$ ramifica se e somente se $p$ dividir o discriminante de $\mathbb{K} / \mathbb{Q}$.

Demonstração: v. [MARCus, 16, p. 72, teorema 24]

1.2.48. Teorema. Seja $\mathbb{Q}(\sqrt{a}) / \mathbb{Q}$, onde $a \in \mathbb{Z} .|a|>1$ e a livre de quadrados, uma extensão quadrática de $\mathbb{Q}$, então o discriminante de $\mathbb{Q}(\sqrt{a})$ sobre $\mathbb{Q}$ é a quando $\alpha \equiv$ $1(\bmod 4)$ ou $4 \cdot a$ quando $a \equiv 2,3(\bmod 4)$.

Demonstração: v. [ENDLER, 3, p. 45] 
1.2.49. Definição. Se $\zeta_{m} \in \mathbb{C}$ é uma raiz $m$-ésima primitiva da unidade, $m \geq 1$, dizemos que $\mathbb{Q}\left(\zeta_{m}\right)$ e seus subcorpos são corpos ciclotômicos.

1.2.50. Proposição. Se $m$ for um inteiro positivo e $\zeta_{m}$ for uma raiz $m$-ésima primitiva da unidade, temos que $\left[\mathbb{Q}\left(\zeta_{m}\right): \mathbb{Q}\right]=\hat{\gamma}(m)$ onde $\hat{\tau}$ é a função de Euler e mais ainda,

$$
\operatorname{Gal}\left(\mathbb{Q}\left(\zeta_{m}\right) / \mathbb{Q}\right) \cong(\mathbb{Z} / m \mathbb{Z})^{*}
$$

Demonstração: Seja $\sigma: \mathbb{Q}^{\prime} \longrightarrow \overline{\mathbb{Q}}$ um homomorfismo que fixe $\mathbb{Q}$. Então $\sigma\left(\zeta_{m}\right)$ também é uma raiz m-ésima da unidade e $\mathbb{Q}^{\prime}\left(\zeta_{m}\right)=\mathbb{Q}^{\prime}\left(\sigma\left(\zeta_{m}\right)\right)$. Portanto, $\sigma\left(\zeta_{m}\right)=\zeta_{m}^{e_{\sigma}}$, onde $\epsilon_{\sigma} \in(\mathbb{Z} / m \mathbb{Z})^{*}$. Claramente a aplicação $\sigma \longmapsto \epsilon_{\sigma}$ é um isomorfismo.

1.2.51. Teorema. Sejam $p$ um primo positivo, $m=p^{n},(n \geq 1), \zeta_{m}$ uma raiz $m$-ésima primitiva da unidade. Então o discriminante de $\mathbb{Q}\left(\zeta_{m}\right)$ é

$$
(-1)^{\frac{p(p-1)}{2}} p^{n+\left(p^{n}\right)-p^{n-1}}
$$

Demonstração: v. [E.NDLER, 3, p. 45]

1.2.52. Teorema. Seja $m$ um inteiro positivo, então o anel de inteiros de $\mathbb{Q}\left(\zeta_{m}\right)$ é $\mathbb{Z}\left[\zeta_{m}\right]$ onde $\zeta_{m}$ é uma raiz primitiva m-ésima da unidade.

Demonstração: v. [ENDLER, 3, p. 35] ou [MARCUS, 16, p. 76, teorema 26]

1.2.53. Teorema. Seja $p$ um primo racional positivo, $\zeta_{p}$ uma raiz p-ésima primitiva da unidade, $\mathbb{Q}\left(\zeta_{p}\right)$, uma extensão ciclotômica de $\mathbb{Q}$ então o ideal $(p)$ se decompõe em $\mathbb{Q}\left(\zeta_{p}\right)$ da seguinte forma:

$$
(p)=\left(\left(1-\zeta_{p}\right)^{p-1}\right)
$$

em $\mathbb{Q}\left(\zeta_{p}\right)$.

Demonstração: v. [ENDLER, 3, p. 35] ou de modo mais geral em [MARCUS, 16, p. 76, teorema 26]

1.2.54. Definição. Seja $\mathbb{L} / \mathbb{K}$ uma extensão finita galoisiana de corpos de números. Dizemos que um ideal primo $\mathfrak{p}_{i} \subset \mathcal{O}_{\mathbb{K}}$ se decompõe completamente em $\mathbb{L}$ se $e_{\mathfrak{P}_{i} / \mathfrak{p}}=1$ e $f_{\mathfrak{P}_{i} / \mathfrak{p}}=1$ para todo primo $\mathfrak{P}_{i}$ que esteja acima de $\mathfrak{p}$. 
1.2.55. Teorema (Lei de decomposição). Sejam $p$ um primo positivo, $m$ um inteiro tal que $p \nmid m$ e $\zeta_{m}$ uma raiz primitiva m-ésima da unidade. Então no anel de inteiros $\mathbb{Z}\left[\zeta_{\mathrm{m}}\right]$, temos

$$
(p)=\mathfrak{p}_{1} \cdots \mathfrak{p}_{g}, \quad N_{Q\left(\zeta_{m}\right) / q}\left(\mathfrak{p}_{i}\right)=p^{f}, \quad f . g=\varphi(m) .
$$

onde $\varphi$ é a função de Euler e $f$ é o menor natural positivo tal que

$$
p^{f} \equiv 1(\bmod m)
$$

Se $p \equiv 1(\bmod m)$, então $p$ se decompõe completamente.

Demonstração: v. [COHN, 2, p. 96, teorema 10.45]

1.2.56. Definição. Sejam $\mathbb{L} / \mathbb{Q}$ uma extensão galoisiana finita, $I_{\mathbb{Z}}$ o grupo dos ideais fracionários em $\mathbb{L}$ e $P_{\mathbb{L}}$ o grupo dos ideais principais de $\mathbb{L}$. Chamamos de grupo de classes o grupo $\frac{I_{\mathbb{L}}}{P_{\mathbb{L}}}$.

1.2.57. Teorema (Kummer). Seja $m$ um inteiro positivo arbitrário. Então cada classe de ideais de $\mathbb{Q}\left(\zeta_{m}\right)$ contém um produto de ideais primos de grau 1.

Demonstração: Uma demonstração desse resultado pode ser encontrado em [HILBERT, 8, pp. 92, 93, 94, "Satz 89"]. 


\subsection{Extensões de grupos}

Os resultados de extensão de grupos, como o teorema de Schur-Zassenhaus, serão usados no capítulo 2, quando falarmos sobre seqüências de grupos exatas que cindem.

1.3.1. Definição. Dizemos que uma seqüência exata curta de grupos

$$
\{1\} \longrightarrow H \longrightarrow \widetilde{G} \underset{\psi}{\stackrel{\varphi}{\rightleftharpoons}} G \longrightarrow\{1\}
$$

cinde se existe um morfismo $\psi: G \longrightarrow \widetilde{G}$ tal que $\varphi \circ \psi=1_{G}$.

1.3.2. Teorema. Sejam $G$ e $H$ dois subgrupos de um grupo $\widetilde{G}$ tais que $H \triangleleft \widetilde{G}, \widetilde{G}=G \cdot H$ e $G \cap H=\{1\}$. Então

$$
\{1\} \longrightarrow H \longrightarrow \widetilde{G} \longrightarrow G \longrightarrow\{1\}
$$

é uma seqüência exata curta de grupos que cinde, se e somente se $\widetilde{G} \cong H \rtimes G$, onde $\rtimes$ significa o produto semidireto de $H$ e $G$, com a ação de conjugação.

Demonstração: v. [Gorestein, 6, p. 26, teorema 5.1] ou [Johnson, 13, p. 127, teorema 2]

1.3.3. Teorema. Se $H$ e $G$ são subgrupos de um grupo $\tilde{G}$ tais que $H \triangleleft \widetilde{G}, H \cap G=\{1\}$ e $H \cdot G=\widetilde{G}$ então $G \cong H \times G$ se e somente se $G$ agir trivialmente sobre $H$.

Demonstração: v. [Gorestein, 6, p. 26, teorema 5.2] ou [Johnson, 13, p. 126, teorema 1]

1.3.4. Teorema (Schur-Zassenhaus). Se $G$ e $H$ são dois grupos finitos tais que $H \triangleleft G$ e $\operatorname{mdc}(|H|,[G: H])=1$ então a seqüência

$$
\{1\} \longrightarrow H \longrightarrow G \longrightarrow G / H \longrightarrow\{1\}
$$

cinde.

Demonstração: v. [GoRESTEIN, 6, p. 221, teorema 2.1] 


\section{Capítulo 2}

\section{Alguns Teoremas Sobre Extensões Centrais}

\subsection{Generalidades}

O objetivo deste capítulo é descrever extensões cíclicas finitas de $\mathbb{Q}$ de grau $m$, fazendo o compositum entre uma extensão cíclica $\mathbb{L}$, e $\mathbb{Q}^{\prime}$. Através de elementos primitivos de $\mathbb{L}^{\prime}:=$ $\mathbb{L} \cdot \mathbb{Q}^{\prime}$ como extensão de $\mathbb{Q}^{\prime}$, encontraremos um elemento primitivo de $\mathbb{L}^{\prime}=\mathbb{Q}^{\prime}(\sqrt[m]{\beta})$ sobre $\mathbb{Q}^{\prime}$ que tem a propriedade $\sigma(\beta)=3^{r(\sigma)} \delta^{m}$, que será descrito por $\Delta(\gamma)=\prod_{\sigma \in G}\left(\sigma^{-1}(\gamma)\right)^{r(\sigma)}$, onde $\gamma \in \mathbb{Q}^{\prime}$ e $\mathbb{Q}^{\prime}(\sqrt[m]{\Delta(\gamma)})=\mathbb{Q}^{\prime}(\sqrt[m]{\beta})$.

O grupo de Galois de $\mathbb{L}^{\prime} / \mathbb{Q}$ é descrito como um produto direto do grupo cíclico $\operatorname{Gal}(\mathbb{L} / \mathbb{Q})$ e $G$.

É conhecido que se $\mathbb{K}$ é um corpo qualquer e $\mathbb{L} / \mathbb{K}\left(\zeta_{m}\right)$ é uma extensão cíclica de grau $m$, então $\mathbb{L}$ é uma extensão que tem a forma $\mathbb{K}\left(\zeta_{m}\right)(\sqrt[m]{\beta})$ onde $\beta \in \mathbb{K}\left(\zeta_{m}\right)$. Isso é conhecido como teoria de Kummer que pode ser estudado, sob um ponto de vista mais geral, em [Artin, 1, pp. 59, 60, 61, 62, 63, 64] ou em [Frölich, 4, p. 89].

2.1.1. Lema. Sejam $a, b \in \mathbb{Q}^{\prime} . S e \mathbb{Q}^{\prime}(\sqrt[m]{a})$ e $\mathbb{Q}^{\prime}(\sqrt[m]{b})$ são duas extensões cíclicas de mesmo grau $m$ sobre $\mathbb{Q}^{\prime}$ então são equivalentes:

(i). $\mathbb{Q}^{\prime}(\sqrt[m]{a})=\mathbb{Q}^{\prime}(\sqrt[m]{b})$;

(ii). $b=a^{r} c^{m}$, onde $c \in \mathbb{Q}^{\prime}$ e $\operatorname{mdc}(r, m)=1,1 \leq r \leq m-1$.

Demonstração: $(i) . \Rightarrow$ (ii). Como $\mathbb{Q}^{\prime}(\sqrt[m]{a})$ é extensão cíclica de $\mathbb{Q}^{\prime}$, tomemos $\sigma$ um gerador de $\operatorname{Gal}\left(\mathbb{Q}^{\prime}(\sqrt[m]{a}) / \mathbb{Q}^{\prime}\right)$, tal que, se $a=\sqrt[m]{a}$, então $\sigma(\alpha)=\zeta_{m} \alpha$ e seja $\beta=\sqrt[m]{b}$. 
Como $\left\{1, a \cdot a^{2} \ldots \ldots a^{m-1}\right\}$ é base de $\mathbb{Q}^{\prime}(a)=\mathbb{Q}^{\prime}(.3)$ sobre $\mathbb{Q}^{\prime}$, temos

$$
3=\sum_{i=1}^{m-1} c_{i} a^{i}
$$

e aplicando $\sigma$ a $\beta$, obtemos

$$
\sigma(\beta)=\zeta_{m}^{r} \beta=\sum_{i=1}^{m-1} c_{i} \zeta_{m}^{i} \alpha^{i},
$$

$\operatorname{com} \operatorname{mdc}(r, m)=1 .^{[6]}$ Conseqüentemente, $\beta=c_{r} \zeta_{m}^{-r} \alpha^{r} \operatorname{com} \operatorname{mdc}(r, m)=1$, de onde concluímos que

$$
b=a^{r} c_{r}^{m}
$$

$\operatorname{com} c \in \mathbb{Q}^{\prime}$ e $\operatorname{mdc}(r, m)=1$.

(ii). $\Rightarrow(i)$. Notemos que $\left[\mathbb{Q}^{\prime}(\sqrt[m]{a}): \mathbb{Q}^{\prime}\right]=\left[\mathbb{Q}^{\prime}(\sqrt[m]{b}): \mathbb{Q}^{\prime}\right]$ e portanto, da inclusão $\mathbb{Q}^{\prime}(\sqrt[m]{b}) \subseteq \mathbb{Q}^{\prime}(\sqrt[m]{a})$, tiramos a igualdade.

2.1.2. Lema. Sejam $\mathbb{L}^{\prime}=\mathbb{Q}^{\prime}(\sqrt[m]{\beta})$, para algum $\beta \in \mathbb{Q}^{\prime}$. Então $\mathbb{L}^{\prime} / \mathbb{Q}$ é galoisiana se e somente se $\forall \sigma \in G, \exists r_{\sigma} \in\{1, \ldots, m-1\}$ tal que $\sigma(\beta)=\beta^{r_{\sigma}} \delta_{\sigma}^{m}$, onde $\delta_{\sigma} \in \mathbb{Q}^{\prime}$ e com $\operatorname{mdc}\left(r_{\sigma}, m\right)=1$.

Demonstração: Se $\mathbb{L}^{\prime} / \mathbb{Q}$ é galoisiana então $\mathbb{L}^{\prime} / \mathbb{Q}^{\prime}$ é galoisiana. Tomando $\sigma \in \operatorname{Gal}\left(\mathbb{L}^{\prime} / \mathbb{Q}^{\prime}\right)$, temos que $\sigma \in \operatorname{Gal}\left(\mathbb{L}^{\prime} / \mathbb{Q}\right)$, pois $\sigma$ fixa $\mathbb{Q}$. Note que $\sigma: \mathbb{L}^{\prime} \rightarrow \overline{\mathbb{Q}}$ e $\sigma(\sqrt[m]{\beta})^{m}=\sigma(\beta)$, ou seja,

$$
\mathbb{Q}^{\prime}(\sqrt[m]{\beta})=\mathbb{Q}^{\prime}(\sigma(\sqrt[m]{\beta}))=\mathbb{Q}^{\prime}(\sqrt[m]{\sigma(\beta)}) .
$$

Aplicando o lema 2.1.1, temos

$$
\sigma(\beta)=\beta^{r_{\sigma}} \delta_{\sigma}^{m}
$$

$\operatorname{com} \operatorname{mdc}\left(r_{\sigma}, m\right)=1$ e $\delta_{\sigma} \in \mathbb{Q}^{\prime}$.

Reciprocamente, se $\sigma(\beta)=\beta^{r} \delta_{\sigma}^{m}, \forall \sigma \in G$ então tomando qualquer monomorfismo $\tau: \mathbb{L}^{\prime} \longrightarrow \overline{\mathbb{Q}}$ que fixa $\mathbb{Q}, \tau\left(\mathbb{Q}^{\prime}\right)=\mathbb{Q}^{\prime}$. Como $\tau(\sqrt[m]{\beta})^{m}=\tau(\beta)=\rho(\beta)=\beta^{r_{\rho}} \delta_{\rho}^{m}$, para algum $\rho \in \operatorname{Gal}\left(\mathbb{Q}^{\prime} / \mathbb{Q}\right)$, com $\operatorname{mdc}\left(r_{\rho}, m\right)=1$, pois $\beta \in \mathbb{Q}^{\prime}$, temos $\tau(\sqrt[m]{\beta})=\sqrt[m]{\beta}{ }^{r} \delta$ para algum $\delta \in \mathbb{Q}^{\prime}$ e $\operatorname{mdc}(r, m)=1$, ou seja, $\tau\left(\mathbb{L}^{\prime}\right)=\mathbb{L}^{\prime}$.

Portanto, acabamos de demonstrar que $\mathbb{L}^{\prime} / \mathbb{Q}$ é uma extensão normal e como $\mathbb{Q}$ é separável, segue que $\mathbb{L}^{\prime}$ é separável sobre $\mathbb{Q}$, de onde segue que $\mathbb{L}^{\prime}$ é extensão galoisiana de $\mathbb{Q}$.

[6] Como $\mathbb{Q}^{\prime}(\alpha)=\mathbb{Q}^{\prime}(\beta)$, temos que $\sigma(\beta)=\zeta_{m}^{r} \beta$, onde $\operatorname{mdc}(r . m)=1$, pois $\sigma$ tem ordem $m$, pois caso tivéssemos $\operatorname{mdc}(r, m) \neq 1$, então existiriam um inteiro $0<k<m$ e um inteiro $u$ tais que $k r=m u$; portanto $\sigma^{k}(\beta)=3$, o que implicaria que $m \mid k$ (absurdo!). 
2.1.3. Definição. Sejam $\mathbb{L}^{\prime}=\mathbb{Q}^{\prime}(\sqrt[m]{3})$, onde is $\in \mathbb{Q}^{\prime *}$, uma extensão galoisiana de $\mathbb{Q}$. Dizemos que $\mathbb{L}^{\prime} / \mathbb{Q}$ é central sobre $\mathbb{L}^{\prime} / \mathbb{Q}^{\prime}$ se $\operatorname{Gal}\left(\mathbb{L}^{\prime} / \mathbb{Q}^{\prime}\right)$ está contido no centro de $\operatorname{Gal}\left(\mathbb{L}^{\prime} / \mathbb{Q}\right)$.

2.1.4. Lema. Seja $\mathbb{L}^{\prime}=\mathbb{Q}^{\prime}(\sqrt[m]{\beta})$, onde $\beta \in \mathbb{Q}^{\prime}$, uma extensão galoisiana de $\mathbb{Q}$. Então $\mathbb{L}^{\prime} / \mathbb{Q}$ é central sobre $\mathbb{L}^{\prime} / \mathbb{Q}^{\prime}$ se e somente se $\sigma(\beta)=\beta^{r(\sigma)} \delta_{\sigma}^{m}$ para todo $\sigma \in G$ e $\delta \in \mathbb{Q}^{\prime}$.

Demonstração: $(\Rightarrow)$ Sejam $H=\operatorname{Gal}\left(\mathbb{L}^{\prime} / \mathbb{Q}^{\prime}\right)$ e $T: H \rightarrow \mu_{m}$, dado por

$$
T(\varphi)=\frac{\varphi(\sqrt[m]{\beta})}{\sqrt[m]{\beta}}
$$

onde $\mu_{m}$ é $\circ$ grupo de todas as raízes $m$-ésimas da unidade.

Notemos que $T$ está bem definida, pois $\varphi \in \operatorname{Gal}\left(\mathbb{L}^{\prime} / \mathbb{Q}^{\prime}\right)$. Assim, se $\sigma \in G$ e $\sigma\left(\zeta_{m}\right)=$ $\left(\zeta_{m}\right)^{r(\sigma)}$, temos que

$$
\sigma(T(\varphi))=T(\varphi)^{r(\sigma)}
$$

Por outro lado,

$$
\sigma(T(\varphi))=\sigma\left(\frac{\varphi(\sqrt[m]{\beta})}{\sqrt[m]{\beta}}\right)=\frac{\tilde{\sigma}(\varphi(\sqrt[m]{\beta}))}{\widetilde{\sigma}(\sqrt[m]{\beta})}
$$

onde $\widetilde{\sigma}$ é qualquer extensão de $\sigma$ a $\operatorname{Gal}\left(\mathbb{L}^{\prime} / \mathbb{Q}\right)$, logo

$$
\frac{\tilde{\sigma}(\varphi(\sqrt[m]{\beta}))}{\tilde{\sigma}(\sqrt[m]{\beta})}=\frac{\varphi(\tilde{\sigma}(\sqrt[m]{\beta}))}{\tilde{\sigma}(\sqrt[m]{\beta})}=\frac{\varphi(\zeta \sqrt[m]{\sigma(\beta)})}{\zeta \sqrt[m]{\sigma(\beta)}}=\frac{\zeta \varphi(\sqrt[m]{\sigma(\beta)})}{\zeta \sqrt[m]{\beta}}=\frac{\varphi(\sqrt[m]{\sigma(\beta)})}{\sqrt[m]{\sigma(\beta)}}
$$

onde $\zeta \in \mu_{m}$, pois $\varphi \in \operatorname{Gal}\left(\mathbb{L}^{\prime} / \mathbb{Q}^{\prime}\right) \subset \mathbb{Z}\left(\operatorname{Gal}\left(\mathbb{L}^{\prime} / \mathbb{Q}\right)\right)$, isto é, $\varphi$ pertence ao centro de $\operatorname{Gal}\left(\mathbb{L}^{\prime} / \mathbb{Q}\right)$, e como $\mathbb{L}^{\prime} / \mathbb{Q}$ é extensão galoisiana e central, temos $\sigma(\beta)=\beta^{r_{\sigma}} \delta_{\sigma}^{m}$, logo

$$
\frac{\varphi(\sqrt[m]{\sigma(\beta)})}{\sqrt[m]{\sigma(\beta)}}=\frac{\varphi\left(\delta_{\sigma} \sqrt[m]{\beta^{r(\sigma)}}\right)}{\delta_{\sigma} \sqrt[m]{\beta^{r(\sigma)}}}=\frac{\delta_{\sigma} \varphi\left(\sqrt[m]{\beta^{r(\sigma)}}\right)}{\delta_{\sigma} \sqrt[m]{\beta^{r(\sigma)}}}=\frac{\varphi\left(\sqrt[m]{\beta^{r_{\sigma}}}\right)}{\sqrt[m]{\beta^{r_{\sigma}}}}=\left(\frac{\varphi(\sqrt[m]{\beta})}{\sqrt[m]{\beta}}\right)^{r_{\sigma}} .
$$

Logo, vale a seguinte igualdade:

$$
\left(\frac{\varphi(\sqrt[m]{\beta})}{\sqrt[m]{\beta}}\right)^{r_{\sigma}}=\left(\frac{\varphi(\sqrt[m]{\beta})}{\sqrt[m]{\beta}}\right)^{r(\sigma)} \forall \sigma \in G \forall \varphi \in H
$$

Variando $\varphi$, é possível obter todas as raízes $m$-ésimas da unidade, em $\frac{\varphi(\sqrt[m]{\beta})}{\sqrt[m]{\beta}}$, portanto, fixando $\sigma \in G, r(\sigma) \equiv r_{\sigma}(\bmod m) \quad \forall \sigma \in G$ e a escolha de $r(\sigma) \in\{0, \ldots, m-1\}$ e $r_{\sigma} \in\{0, \ldots, m-1\}$, segue $r(\sigma)=r_{\sigma}$.

Reciprocamente, notemos que

$$
\mathbb{L}^{\prime}=\mathbb{Q}\left(\zeta_{m}, \sqrt[m]{\beta}\right)
$$


Portanto basta mostrar que $\tau$ o $\sigma=\sigma \circ \tau \forall \tau \in \operatorname{Gal}\left(\mathbb{L}^{\prime} / \mathbb{Q}^{\prime}\right)$ e $\forall \sigma \in \operatorname{Gal}\left(\mathbb{L}^{\prime} / \mathbb{Q}\right)$, apenas nos elementos $\zeta_{m}$ e $\sqrt[m]{\beta}$. De fato, se $\tau \in \operatorname{Gal}\left(\mathbb{L}^{\prime} / \mathbb{Q}^{\prime}\right)$ e $\sigma \in \operatorname{Gal}\left(\mathbb{L}^{\prime} / \mathbb{Q}\right)$, então

$$
\tau \sigma\left(\zeta_{m}\right)=\tau\left(\zeta_{m}^{r(\sigma)}\right)=\zeta_{m}^{r(\sigma)}
$$

pois $\zeta_{m} \in \mathbb{Q}^{\prime}$ e portanto é fixo por $\tau$. Por outro lado,

$$
\sigma \tau\left(\zeta_{m}\right)=\sigma\left(\zeta_{m}\right)=\zeta_{m}^{r(\sigma)}
$$

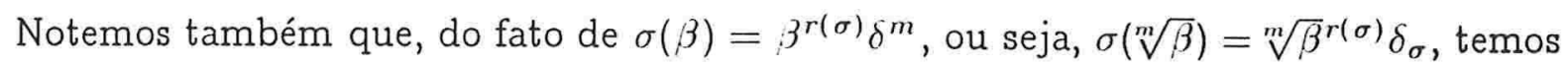

$$
\tau(\sigma(\sqrt[m]{\beta}))=\tau\left(\sqrt[m]{\beta}^{r(\sigma)} \delta_{\sigma}\right)=\sqrt[m]{\beta}^{r(\sigma)} \zeta_{m}^{t_{r} r(\sigma)} \delta_{\sigma}
$$

e que

$$
\sigma(\tau(\sqrt[m]{\beta}))=\sigma\left(\zeta_{m}^{t_{r} m} \sqrt{\beta}\right)=\zeta_{m}^{t_{r} r(\sigma)} \sqrt[m^{m}]{\beta} r(\sigma) \delta_{\sigma}
$$

portanto $\mathbb{L}^{\prime} / \mathbb{Q}$ é central sobre $\mathbb{L}^{\prime} / \mathbb{Q}^{\prime}$.

2.1.5. Lema. Seja $\mathbb{L}^{\prime}=\mathbb{Q}^{\prime}(\sqrt[m]{\beta})$, onde $\beta \in \mathbb{Q}^{\prime}$, uma extensão galoisiana de $\mathbb{Q}$. Sejam $H=\operatorname{Gal}\left(\mathbb{L}^{\prime} / \mathbb{Q}^{\prime}\right)$ e $\widetilde{G}=\operatorname{Gal}\left(\mathbb{L}^{\prime} / \mathbb{Q}\right)$. Então

$$
\{1\} \longrightarrow H \longrightarrow \widetilde{G} \longrightarrow G \longrightarrow\{1\}
$$

é central e cinde se e somente se existe uma extensão cíclica $\mathbb{L} / \mathbb{Q}$ tal que $\mathbb{L} \cap \mathbb{Q}^{\prime}=\mathbb{Q}$ e $\mathbb{L}^{\prime}=\mathbb{L} \cdot \mathbb{Q}^{\prime}$.

Demonstração: Se a seqüência

$$
\{1\} \longrightarrow H \stackrel{\psi}{\longrightarrow} \widetilde{G} \stackrel{\varphi}{\longrightarrow} G \longrightarrow\{1\}
$$

cinde, seja $\nu: G \rightarrow \widetilde{G}$ a cessão. Tomemos $\mathbb{L}=\left(\mathbb{L}^{\prime}\right)^{\nu(G)}$, onde $\left(\mathbb{L}^{\prime}\right)^{\nu(G)}$ é o corpo fixo pelo grupo $\nu(G)$. Precisamos mostrar que $\mathbb{L}^{\prime}=\mathbb{L} \cdot \mathbb{Q}^{\prime}$ e $\mathbb{Q}=\mathbb{L} \cap \mathbb{Q}^{\prime}$.

Como a seqüência cinde, $\operatorname{Gal}\left(\mathbb{L}^{\prime} / \mathbb{Q}\right)$ é isomorfo ao produto semidireto de $H$ e $G$.

Logo $\psi\left(\operatorname{Gal}\left(\mathbb{L}^{\prime} / \mathbb{Q}^{\prime}\right)\right) \cap \nu\left(\operatorname{Gal}\left(\mathbb{Q}^{\prime} / \mathbb{Q}\right)\right)=\left\{1_{\widetilde{G}}\right\}$, ou seja,
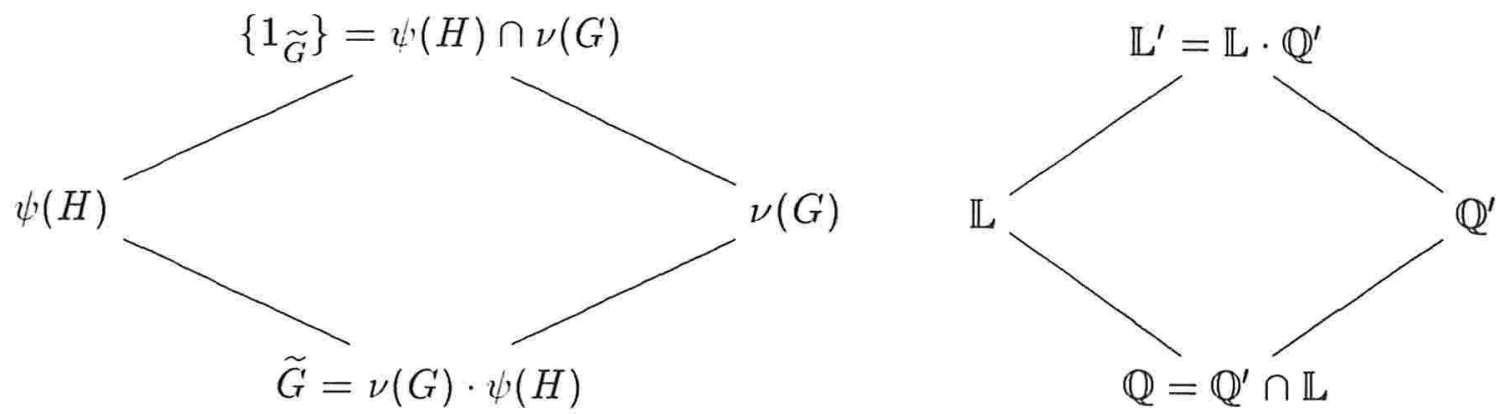
e pelo teorema fundamental da teoria de Galois, valem $\mathbb{L} \cap \mathbb{Q}^{\prime}=\mathbb{Q}$ e $\mathbb{L} \cdot \mathbb{Q}^{\prime}=\mathbb{L}^{\prime}$ e como $\mathbb{L}^{\prime} / \mathbb{Q}^{\prime}$ é uma extensão cíclica, temos que $\mathbb{L} / \mathbb{Q}$ também é um extensão cíclica.

Reciprocamente, se existe uma extensão cíclica de $\mathbb{L}$ de $\mathbb{Q}$, tal que $\mathbb{L}^{\prime}=\mathbb{Q}^{\prime} \cdot \mathbb{L}$ e $\mathbb{L} \cap \mathbb{Q}^{\prime}=\mathbb{Q}$, o grupo $\operatorname{Gal}\left(\mathbb{L}^{\prime} / \mathbb{Q}\right)$ é isomorfo ao produto direto de $\operatorname{Gal}\left(\mathbb{L}^{\prime} / \mathbb{Q}^{\prime}\right) \operatorname{com} \operatorname{Gal}\left(\mathbb{Q}^{\prime} / \mathbb{Q}\right)$ e tal isomorfismo é dado pela restrição. Como o grupo $\mathrm{Gal}(\mathbb{L} / \mathbb{Q})$ é cíclico, temos que $\mathbb{L}^{\prime} / \mathbb{Q}^{\prime}$ será sempre uma extensão cíclica, pois $\operatorname{Gal}\left(\mathbb{L}^{\prime} / \mathbb{Q}^{\prime}\right) \cong \operatorname{Gal}(\mathbb{L} / \mathbb{Q})$ e a seqüência $\{1\} \longrightarrow H \longrightarrow \widetilde{G} \longrightarrow G \longrightarrow\{1\}$ cinde.

2.1.6. Lema. Se $m \geq 3, \mathbb{L}^{\prime}=\mathbb{Q}^{\prime}(\sqrt[m]{\beta})$, onde $\beta \in \mathbb{Q}^{\prime}$, é uma extensão galoisiana de $\mathbb{Q}$, $H=\operatorname{Gal}\left(\mathbb{L}^{\prime} / \mathbb{Q}^{\prime}\right)$ e $\widetilde{G}=\operatorname{Gal}\left(\mathbb{L}^{\prime} / \mathbb{Q}\right)$ e a extensão de grupos

$$
\{1\} \longrightarrow H \longrightarrow \widetilde{G} \longrightarrow G \longrightarrow\{1\}
$$

é central e cinde então a norma de $\beta$, em relação a $\mathbb{Q}^{\prime} / \mathbb{Q}$, é uma potência $m$-ésima de um elemento de $\mathbb{Q}$.

Demonstração: Se a seqüência for central e cindir então $\sigma(\beta)=\beta^{r(\sigma)} \delta_{\boldsymbol{\sigma}}^{m}$, para todo $\sigma \in \operatorname{Gal}\left(\mathbb{Q}^{\prime} / \mathbb{Q}\right)$. Portanto

$$
N_{Q^{\prime} / \mathbb{Q}}(\beta)=\prod_{\sigma \in G} \sigma(\beta)=\prod_{\sigma \in G} \beta^{r(\sigma)} \delta_{\sigma}^{m}
$$

Como $\operatorname{Gal}\left(\mathbb{Q}^{\prime} / \mathbb{Q}\right) \cong \mathbb{Z}_{m}^{*}$, então $s:=\sum_{\sigma \in G} r(\sigma) \equiv 0(\bmod m)$, logo

$$
N_{0 Q^{\prime Q Q}}(\beta)=\prod_{\sigma \in G} \beta^{r(\sigma)} \delta_{\sigma}^{m}=\beta^{s} \prod_{\sigma \in G} \delta_{\sigma}^{m}=\left(\beta^{k}\right)^{m}\left(\prod_{\sigma \in G} \delta_{\sigma}\right)^{m}=\delta^{m},
$$

onde $\delta \in \mathbb{Q}^{\prime}$.

Seja $U$ o conjunto $\nu(G)$, onde $\nu: G \longrightarrow \widetilde{G}$ é a cessão, e consideremos o seguinte produto:

$$
b=\prod_{\bar{\sigma} \in U} \bar{\sigma}(\sqrt[m]{\beta})
$$

que claramente está em $\mathbb{L}^{\prime}$. Notemos também que $b^{m}=\delta^{m}$, e portanto $b$ difere de $\delta$ por uma raiz $m$-ésima da unidade, o que implica que $b \in \mathbb{Q}^{\prime}$.

Se $\tau \in G$, consideremos $\bar{\tau}=\nu(\tau)$ então

$$
\tau(b)=\tau\left(\prod_{\bar{\sigma} \in U} \bar{\sigma}(\sqrt[m]{\beta})\right)=\prod_{\bar{\sigma} \in U} \bar{\tau}(\bar{\sigma}(\sqrt[m]{\beta}))=\prod_{\bar{\sigma} \in U} \bar{\sigma}(\sqrt[m]{\beta})=b
$$

$b$ é fixo por $\tau, \forall \tau \in G$, o que implica que $b \in \mathbb{Q}$. 
Queremos elementos em $\beta \in \mathbb{Q}^{\prime}$ tais que $\sigma(\beta)=\beta^{r(\sigma)} \delta_{\sigma}^{m}$. Notemos que sempre temos, para os elementos primitivos de $\mathbb{L}^{\prime} / \mathbb{Q}^{\prime}, \sigma(\beta)=\beta^{r_{\sigma}} \delta_{\sigma}^{m}$, para algum $\delta_{\sigma} \in \mathbb{Q}^{\prime}$, mas nem sempre $\sigma(\beta)=\beta^{r(\sigma)} \delta_{\sigma}^{m}$.

Consideremos em $\mathbb{Z}[G]$ (o anel de grupo de $G$ ), o seguinte elemento:

$$
\Delta=\sum_{\sigma \in G} r(\sigma) \sigma^{-1}
$$

onde $r: G \longrightarrow(\mathbb{Z} / m \mathbb{Z})^{*}$ é o isomorfismo dado por

$$
\sigma\left(\zeta_{m}\right)=\zeta_{m}^{r(\sigma)} \forall \sigma \in G, r(\sigma) \in\{1,2, \ldots, m\}
$$

Alguns exemplos de $\Delta$, identificando $G \operatorname{com}(\mathbb{Z} / m \mathbb{Z})^{*}$, temos:

$\mathrm{m}=2 \Delta=1$

$\mathrm{m}=3 \Delta=1 \cdot 1+2 \cdot r\left(2^{-1}\right)=1+4=5 ;$

$\mathrm{m}=4 \Delta=1 \cdot 1+3 \cdot r\left(3^{-1}\right)=1+9=10 ;$

$\mathrm{m}=5 \Delta=1 \cdot 1+2 \cdot r\left(2^{-1}\right)+3 \cdot r\left(3^{-1}\right)+4 \cdot r\left(4^{-1}\right)=1+2 \cdot 3+4 \cdot 4+2 \cdot 3=1+6+16+6=29$.

Para $\Delta$, é fácil ver que vale a seguinte congruência:

$$
\tau \Delta \equiv r(\tau) \Delta(\bmod m \mathbb{Z}[G])
$$

De fato, de $\Delta=\sum_{\sigma \in G} r(\sigma) \sigma^{-1}$ temos

$$
\begin{gathered}
\tau \Delta=\sum_{\sigma \in G} \tau r(\sigma) \sigma^{-1}=\sum_{\sigma \in G} r(\sigma) \tau \sigma^{-1}=\sum_{\sigma \in G} r\left(\sigma \tau^{-1} \tau\right)\left(\sigma \tau^{-1}\right)^{-1} \\
\equiv r(\tau) \sum_{\sigma \in G} r\left(\sigma \tau^{-1}\right)\left(\sigma \tau^{-1}\right)^{-1}=r(\tau) \sum_{\sigma \in G} r(\sigma) \sigma^{-1}(\bmod m \mathbb{Z}[G]) .
\end{gathered}
$$

Definimos a ação de um elemento, $\theta=\sum_{\sigma \in G} a_{\sigma} \sigma$ em $\gamma \in \mathbb{Q}^{\prime}$, do seguinte modo:

$$
\theta(\gamma)=\prod_{\sigma \in G}(\sigma(\gamma))^{a_{\sigma}}
$$

desse modo, a ação de $\Delta$ em $\gamma \in \mathbb{Q}^{\prime}$ é

$$
\Delta(\gamma)=\prod_{\sigma \in G}\left(\sigma^{-1}(\gamma)\right)^{r(\sigma)} .
$$

De $(*)$, concluímos que se $\sigma \in G$ então

$$
\sigma(\Delta(\gamma))=(\Delta(\gamma))^{r(\sigma)} \delta_{\sigma}^{m} .
$$


2.1.7. Lema. Se $\mathbb{L}^{\prime}=\mathbb{Q}^{\prime}(\sqrt[4]{\beta}),\left(\beta \in \mathbb{Q}^{\prime}\right)$ é extensão galoisiana de $\mathbb{Q}$ então $\mathbb{L}^{\prime} / \mathbb{Q}$ é central sobre $\mathbb{L}^{\prime} / \mathbb{Q}^{\prime}$ se e somente se

$$
\mathbb{L}^{\prime}=\mathbb{Q}^{\prime}\left(\sqrt[4]{\Delta(\imath) \delta^{2}}\right)
$$

$\operatorname{com} \gamma, \delta \in \mathbb{Q}^{\prime}$ e $\delta^{2} \in \mathbb{Q}$.

Demonstração: Sejam $H=\operatorname{Gal}\left(\mathbb{L}^{\prime} / \mathbb{Q}^{\prime}\right), \widetilde{G}=\operatorname{Gal}\left(\mathbb{L}^{\prime} / \mathbb{Q}\right)$ e $r(G)=\{-1,1(\bmod 4)\}$.

Se $\sigma \in G_{1}, \sigma \neq 1_{G}$, então

$$
\Delta=1_{G_{i}}+3 \sigma=1_{G}-\sigma+4 \sigma,
$$

pois $r(G)=\{1,-1(\bmod 4)\}$. Do fato de $\mathbb{L}^{\prime} / \mathbb{Q}$ ser central sobre $\mathbb{L}^{\prime} / \mathbb{Q}^{\prime}$, temos $\sigma(\beta)=$ $\beta^{-1} \delta_{\sigma}^{4}$, ou seja,

$$
N_{Q / \mathcal{Q}}\left(\beta^{2} \delta^{-4}\right)=\beta^{2} \delta^{-4} \sigma(\beta) \delta^{-4}=\beta^{2} \sigma(\beta)^{2} \delta^{-4} \sigma(\delta)^{-4}=\delta^{8} \delta^{-8}=1,
$$

como a norma é multiplicativa, temos

$$
N_{Q^{\prime} / Q^{\prime}}\left(\beta \delta^{-2}\right)^{2}=1 \Rightarrow N_{Q / Q}\left(\beta \delta^{-2}\right)= \pm 1 \text {. }
$$

Do fato da norma de qualquer elemento de $\mathbb{Q}^{\prime}$ não poder ser negativa, pois tal norma é soma de dois quadrados, temos $N_{Q^{\prime} / Q}\left(B \delta^{-2}\right)=1$.

Pelo "Satz-90" de Hilbert ${ }^{[i]}$, existe $\gamma \in \mathbb{Q}^{\prime *}$, tal que

$$
\beta \delta^{-2}=\frac{\gamma}{\sigma(\gamma)}
$$

em que $\sigma$ é um gerador de $G$. Como

$$
N_{Q^{\prime} / Q^{\prime}}\left(\beta \delta^{-2}\right)=1
$$

temos

$$
\beta \sigma(\beta) \delta^{-2} \sigma\left(\delta^{-2}\right)=1,
$$

porém $\beta \sigma(\beta)=\delta^{4}, \log$ o, $\sigma\left(\delta^{2}\right)=\delta^{2}$, ou seja, $\delta^{2} \in \mathbb{Q}$, e de

$$
\Delta(\gamma)=\frac{\gamma}{\sigma(\gamma)} \sigma(\gamma)^{4}
$$

temos

$$
\beta=\frac{\delta^{2} \Delta(\gamma)}{\sigma(\gamma)^{4}}
$$

Reciprocamente se $\mathbb{L}^{\prime}=\mathbb{Q}^{\prime}\left(\sqrt[4]{\left.\delta^{2}\right\lrcorner(\hat{\jmath})}\right)$ e $\delta^{2} \in \mathbb{Q}$, então para mostrar que $\mathbb{L}^{\prime} / \mathbb{Q}$ é central sobre $\mathbb{L}^{\prime} / \mathbb{Q}^{\prime}$, basta calcular $\sigma(\beta)$ para $\beta=\Delta(\gamma) \delta^{2}$.

[7] Cf. Capítulo 1, teorema 1.1.3. 
Mas

$$
\sigma(\Delta(\vartheta))=\left(\Delta\left(\imath_{i}\right)\right)^{3} u^{4}
$$

portanto

$$
\sigma(\beta)=(\Delta(\gamma))^{3} u^{4} \sigma\left(\delta^{2}\right)=\beta^{3} u^{4} \delta^{-4},
$$

pois $\delta^{2} \in \mathbb{Q}$. Portanto pelo lema 2.1.3, $\mathbb{L}^{\prime} / \mathbb{Q}$ é central.

O caso em que $\ell=2^{n}$ são diferenciados do caso em que $\ell$ é um primo ímpar, porque precisamos mostrar a existência de um elemento primitivo de $\mathbb{Z} / m \mathbb{Z}$, onde $m=\ell^{n}$. Sabemos que $\left(\mathbb{Z} / 2^{n} \mathbb{Z}\right)^{*}$ é isomorfo ao produto de um grupo cíclico de ordem $2^{n-2}$ por outro de grau 2.

2.1.8. Lema. Sejam $\ell \neq 2$ um primo, $m=\ell^{n} e \mathbb{L}^{\prime}=\mathbb{Q}^{\prime}(\sqrt[m]{\beta})$, onde $\beta \in \mathbb{Q}^{\prime}$, uma extensão galoisiana de $\mathbb{Q}$ então $\mathbb{L}^{\prime} / \mathbb{Q}$ é central sobre $\mathbb{L}^{\prime} / \mathbb{Q}^{\prime}$ se e somente se

$$
\mathbb{L}^{\prime}=\mathbb{Q}^{\prime}(\sqrt[m]{\Delta(\gamma) \zeta})
$$

onde $\gamma \in \mathbb{Q}^{\prime}$ e $\zeta^{m}=1$.

Demonstração: Seja $f=|G|$, ou seja, $f=\varphi\left(\ell^{n}\right)=\ell^{n-1}(\ell-1)$. Lembrando que $G \stackrel{r}{\longrightarrow} \mathbb{Z}_{m}^{*}$, é um isomorfismo e como $C \neq 2$, existe um elemento $g$ que gera $\mathbb{Z}_{m}^{*}$. Sempre é possível escolher $g$ em $\mathbb{Z}_{m}^{*}$ tal que

$$
g^{f} \equiv 1\left(\bmod \ell^{n}\right) \quad \text { e } \quad g^{f} \not \equiv 1\left(\bmod \ell^{n+1}\right) .
$$

De fato, se $g_{1}$ gera $\mathbb{Z}_{\ell}^{*}$, então podem ocorrer: $\left\{\begin{array}{l}g_{1}^{\ell-1} \neq 1\left(\bmod \ell^{2}\right) \\ \text { ou } \\ g_{1}^{\ell-1} \equiv 1\left(\bmod \ell^{2}\right)\end{array}\right.$.

No segundo caso, escolhe-se $g_{1}+\ell$ como gerador. Naturalmente, a possibilidade de escolha pode ser generalizada, seja

$$
g_{n}=g_{1}^{\ell^{n-1}}(1+\ell) \quad \text { para } n \geq 2
$$

então

$$
g_{n}^{\ell^{n-1}(\ell-1)} \equiv 1\left(\bmod \ell^{n}\right)
$$

e

$$
g_{n}^{\ell^{n-1}(\ell-1)} \equiv(1+\ell)^{\ell^{n-1}(\ell-1)} g_{1}^{\ell^{2^{n}-2}(\ell-1)} \equiv(1+\ell)^{\ell^{n-1}(\ell-1)} \not \equiv 1\left(\bmod \ell^{n-1}\right)
$$

caso $g_{n}$ não satisfaça as condições dadas.

Logo existe um elemento $\sigma$ em $G$ tal que $\sigma\left(\zeta_{m}\right)=\zeta_{m}^{g}$. Seja $\bar{\sigma}$ qualquer extensão de $\sigma$ a $\operatorname{Gal}\left(\mathbb{L}^{\prime} / \mathbb{Q}\right)$, calculando $\bar{\sigma}(\sqrt[m]{\beta})$, obtemos

$$
\bar{\sigma}(\sqrt[m]{\beta})=\zeta \sqrt[m]{\sigma(\beta)}=\sqrt[m]{\beta}{ }^{g} \delta
$$

para algum $\delta \in \mathbb{Q}^{\prime}$, por outro lado, $\bar{\sigma}(\bar{\sigma}(\sqrt[m]{\beta}))=\sqrt[m]{\beta^{g^{2}}} \delta^{g} \sigma(\delta)$. 
Dessa forma:

$$
\bar{\sigma}^{f}(\sqrt[m]{\beta})=\sqrt[m]{3^{g^{j}}} \delta^{g^{f-1}} \sigma\left(\delta^{g^{j-2}}\right) \sigma^{2}(\delta)^{g^{f-3}} \cdots \sigma^{f-1}(\delta)=\sqrt[m]{\beta^{g^{j}}} \Delta(\sigma(\delta)) .
$$

Isso porque vale a seguinte relação de congruência:

$$
r(\sigma) \sigma \equiv g^{-1} \sigma(\bmod m \mathbb{Z}[G])
$$

pois sendo $\sigma$ a conjugação, $\sigma\left(\zeta_{m}\right)=\zeta_{m}^{-1}$, e portanto,

$$
\sum_{\sigma \in G} r\left(\sigma^{-1}\right) \sigma \equiv \sum_{i=1}^{f} g^{-i} \sigma^{i}(\bmod m \mathbb{Z}[G]) .
$$

Portanto $\sqrt[m]{\beta^{g^{\prime}}}=\sqrt[m]{\beta} \beta^{q}$, onde $\ell \chi q$, pois

$$
g^{f} \equiv 1\left(\bmod \ell^{n}\right) \quad \text { e } \quad g^{f} \not \equiv 1\left(\bmod \ell^{n+1}\right) .
$$

Logo,

$$
\bar{\sigma}^{f}(\sqrt[m]{\beta})=\sqrt[m]{\beta} \Delta(\sigma(\delta)) \beta^{q}
$$

$\operatorname{com} C X q$.

$\operatorname{Mas} \bar{\sigma}^{f} \in \operatorname{Gal}\left(\mathbb{L}^{\prime} / \mathbb{Q}^{\prime}\right)$, pois

$$
\bar{\sigma}^{f}\left(\zeta_{m}\right)=\zeta_{m}^{g^{\prime}}=\zeta_{m}
$$

de onde $\mathbb{Q}^{\prime}$ fica fixo por $\bar{\sigma}^{f}$, portanto

$$
\bar{\sigma}^{f}(\sqrt[m]{\beta})=\sqrt[m]{\beta} \zeta
$$

onde $\zeta$ é uma raiz $m$-ésima da unidade. Pelo fato de $\mathbb{L}^{\prime} / \mathbb{Q}^{\prime}$ ser galoisiana, temos

$$
\mathbb{L}^{\prime}=\mathbb{Q}^{\prime}\left(\sqrt[m]{\beta^{q}}\right)
$$

porque $\mathbb{L}^{\prime} / \mathbb{Q}^{\prime}$ é galoisiana e $\ell \nmid q$;

$$
\mathbb{Q}^{\prime}\left(\sqrt[m]{\beta^{q}}\right)=\mathbb{Q}^{\prime}\left(\sqrt[m]{\Delta(\sigma(\delta)) \zeta_{m}^{+}}\right)
$$

pois

$$
\mathbb{Q}^{\prime}\left(\sqrt[m]{\beta^{q}}\right)=\mathbb{Q}^{\prime}\left(\sqrt[m]{\beta^{-q}}\right)=\mathbb{Q}^{\prime}\left(\sqrt[m]{\Delta(\sigma(\delta)) \zeta^{-1}}\right)
$$

Reciprocamente, se

$$
\mathbb{L}^{\prime}=\mathbb{Q}^{\prime}(\sqrt[m]{\beta})=\mathbb{Q}^{\prime}\left(\sqrt[m]{\Delta(\sigma(\delta)) \zeta_{m}^{t}}\right)
$$

para mostrar que $\mathbb{L}^{\prime} / \mathbb{Q}$ é central sobre $\mathbb{L}^{\prime} / \mathbb{Q}^{\prime}$, basta calcular $\tau(\beta)$ onde $\beta=\Delta(\sigma(\delta)) \zeta_{m}^{t}$ e $\tau \in \operatorname{Gal}\left(\mathbb{Q}^{\prime} / \mathbb{Q}\right)$.

Como

$$
\tau(\Delta(\sigma(\delta)))=\Delta(\sigma(\delta))^{r(\tau)} u^{m} \quad \text { e } \quad \tau\left(\zeta_{m}^{t}\right)=\zeta_{m}^{t . r(\tau)}
$$

então

$$
\tau(\beta)=\beta^{r(\tau)} k^{m}
$$

e pelo lema 2.1.3 $\mathbb{L}^{\prime} / \mathbb{Q}$ é galoisiana e central sobre $\mathbb{L}^{\prime} / \mathbb{Q}^{\prime}$. 
2.1.9. Corolário. Sejam ( um primo, $\mathbb{L} / \mathbb{Q}$ qualquer extensão cíclica de grau ( e $\mathbb{Q}^{\prime}=$ $\mathbb{Q}\left(\zeta_{\ell}\right)$. Então para o compositum $\mathbb{L}^{\prime}=\mathbb{Q}^{\prime} \cdot \mathbb{L}$ vale $\mathbb{L}^{\prime}=\mathbb{Q}^{\prime}(\sqrt[l]{\Delta(\gamma)})$ para algum $\gamma \in \mathbb{Q}^{\prime}$.

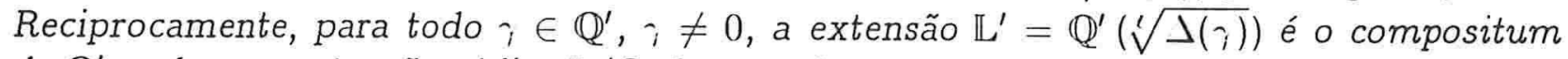
de $\mathbb{Q}^{\prime}$ e alguma extensão cíclica $\mathbb{L} / \mathbb{Q}$ de grau (.

Demonstração: $O$ caso $l^{\prime}=2$ é claro. Suponhamos que $\ell \neq 2$. Seja $\widetilde{\mathbb{Q}}:=\mathbb{L} \cap \mathbb{Q}^{\prime}$ então

$$
[\mathbb{L}: \widetilde{\mathbb{Q}}] \cdot[\widetilde{\mathbb{Q}}: \mathbb{Q}]=C,
$$

mas $\ell$ é um primo, de onde

$$
[\mathbb{L}: \widetilde{\mathbb{Q}}]=1 \quad \text { ou } \quad[\mathbb{L}: \widetilde{\mathbb{Q}}]=\ell \text {. }
$$

Dos fatos $\widetilde{\mathbb{Q}} \subseteq \mathbb{Q}^{\prime},\left[\mathbb{Q}^{\prime}: \widetilde{\mathbb{Q}}\right] \cdot[\widetilde{\mathbb{Q}}: \mathbb{Q}]=\ell-1,[\widetilde{\mathbb{Q}}: \mathbb{Q}]$ ser um divisor de $\ell$ e $\ell-1$, tiramos então, $[\widetilde{\mathbb{Q}}: \mathbb{Q}]=1$. Pelos lemas 2.1 .8 e 2.1 .5 a extensão cinde e

$$
\mathbb{L}^{\prime}=\mathbb{Q}^{\prime}(\sqrt[\ell]{\Delta(\gamma)})
$$

Reciprocamente, se

$$
\mathbb{L}^{\prime}=\mathbb{Q}^{\prime}(\sqrt[l]{\Delta(\gamma)})
$$

então a seqüência

$$
\{1\} \longrightarrow H \longrightarrow \widetilde{G} \longrightarrow G \longrightarrow\{1\}
$$

é exata e central. Como $\ell$ é um primo, $H$ tem ordem $\ell, G$ tem ordem $\ell-1$ e $H \subset \mathbb{Z}(\widetilde{G})$, pelo teorema de Schur-Zassenhaus ${ }^{[8]}$, a seqüência

$$
\{1\} \longrightarrow H \longrightarrow \widetilde{G} \longrightarrow G \longrightarrow\{1\}
$$

cinde e pelo lema 2.1.5, existe uma extensão cíclica tal que $\mathbb{L}^{\prime}=\mathbb{L} \cdot \mathbb{Q}^{\prime}$.

[8] Cf. Capítulo 1, teorema 1.3.4. 


\section{Capítulo 3}

\section{Alguns Resultados Sobre Corpos Ciclotômicos}

\subsection{Caracteres sobre corpos finitos}

O objetivo deste capítulo é demonstrar o teorema de Kummer sobre somas de Gauss e enunciar sua generalização, o teorema de Weber, que serão usados no capítulo 4.

Quanto ao grupo de Galois de $\mathbb{Q}\left(\zeta_{m}\right) / \mathbb{Q}$ temos o isomorfismo:

$$
\operatorname{Gal}\left(\mathbb{Q}\left(\zeta_{m}\right) / \mathbb{Q}\right) \stackrel{\cong}{\longrightarrow}(\mathbb{Z} / m \mathbb{Z})^{*}
$$

dado por $\sigma \longmapsto r(\sigma)$ onde $\sigma\left(\zeta_{m}\right)=\zeta_{m}^{r(\sigma)} \cdot[9]$

Seja $f: \mathbb{F}_{q} \longrightarrow \Omega$ uma função de $\mathbb{F}_{q}$ a valores num corpo algebricamente fechado de característica zero $\Omega$. A Transformada de Fourier de $f$ é definida por:

$$
T f(y)=\sum_{x \in \mathbb{F}_{q}^{*}} f(x) \lambda(-x y)=\sum_{x \in \mathbb{F}_{q}^{*}} f(x) \zeta^{-T r(x y)} .
$$

[9] Cf. Capítulo 1, proposição 1.2.50. 
3.1.1. Teorema. Seja $f^{-}$a função definida por $f^{-}(x):=f(-x)$. Então

(a). $T^{2} f=q f^{-}$.

(b). se definirmos a convolução das funções $f, g: \mathbb{F}_{q} \longrightarrow \Omega$, por:

$$
f * g(y)=\sum_{x \in \mathbb{F}_{q}} f(x) g(y-x)
$$

então $T(f * g)=(T f)(T g)$.

(c). $T(f . g)=\frac{1}{q} T f * T g$.

Demonstração: Verificação direta.

3.1.2. Definição. A soma de Gauss, $S(\chi, \lambda)$, é definida por:

$$
S(\chi, \lambda)=S(\chi)=\sum_{x \in \mathbb{F}^{*}} \backslash(x) \lambda(x) .
$$

Para as somas de Gauss valem:

3.1.3. Proposição. Para todo o caracter $\chi$ não trivial, temos $T(\chi)=\chi(-1) S(\chi) \chi^{-1}$. Demonstração: Por definição

$$
T \chi(y)=\sum_{x \in \mathbb{F}_{q}^{*}} \chi(x) \lambda(-y x)
$$

Se $y=0$, então é claro que $T_{\chi}(y)=0$.

Se $y \neq 0$, considere $x=-t / y$, então

$$
T \chi(y)=\sum_{t \in \mathbb{F}_{q}^{*}} \chi(-t / y) \lambda(t)=\chi(-1) \sum_{t \in \mathbb{F}_{q}^{*}} \chi(t) \lambda(t) \lambda(y)^{-1}
$$

3.1.4. Proposição. Se $\chi$ for o caracter trivial então $S(\chi)=-1$.

Demonstração: Verificação direta. 
3.1.5. Proposição. $S(\bar{\Upsilon})=\backslash(-1) \overline{S(\backslash)}$, onde $\overline{S(\backslash)}$ é o conjugado de $S(\backslash)$, e se \ não for o caracter trivial então $S(\uparrow) S(\bar{\Upsilon})=\backslash(-1) q$.

Demonstração: Notemos que

$$
T^{2}(\curlywedge)=T\left(\backslash(-1) S(\backslash)^{-1}\right)=\backslash(-1) S(\curlywedge) T\left(\curlywedge^{-1}\right)=S(\curlywedge) S\left(\chi^{-1}\right) \chi .
$$

Mas pelo teorema 3.1.1, $T^{2} \gamma=T^{-}$.

No caso $\Omega=\mathbb{C}$, chegamos a uma importante equação:

$$
|S(x)|=q^{1 / 2}
$$

3.1.6. Definição. A soma de Jacobi é definida por:

$$
J\left(\chi_{1}, \lambda_{2}\right)=-\sum_{x \in \mathbb{F}_{q}^{*}} \backslash_{1}(x) \chi_{2}(1-x)=-\left(\chi_{1} * \chi_{2}\right)(1)
$$

onde $\chi_{1}$ e $\chi_{2}$ são caracteres multiplicativos de de $\mathbb{F}_{q}^{*}$ sobre $\mathbb{C}$.

É fácil ver que $J(\mathbf{1}, \mathbf{1})=-(q-2)$, onde 1 representa o caracter trivial. Além disso:

3.1.7. Proposição. Se $\chi_{1} \chi_{2}$ não for o caracter trivial então

$$
J\left(\chi_{1}, \chi_{2}\right)=-\frac{S\left(\chi_{1}\right) S\left(\chi_{2}\right)}{S\left(\chi_{1} \chi_{2}\right)} .
$$

Em particular, $J\left(\mathbf{1}, \chi_{2}\right)=J\left(\chi_{1}, \mathbf{1}\right)=1$. Se $\chi_{1} \chi_{2}=1$ e pelo menos um $\chi_{i}$ for não trivial, então $J\left(\chi_{1}, \lambda_{2}\right)=\chi_{i}(-1)$.

Demonstração: Como $J\left(\chi_{1}, \chi_{2}\right)=-\left(\chi_{1} * \chi_{2}\right)(1)$,

$$
T\left(-\left(\chi_{1} * \curlywedge_{2}\right)\right)=-T\left(\chi_{1} * \chi_{2}\right)=-\left(T \backslash_{1}\right)\left(T \chi_{2}\right)=-\chi_{1}(-1) S\left(\chi_{1}\right) \chi_{1}^{-1} \chi_{2}(-1) S\left(\chi_{2}\right) \chi_{2}^{-1},
$$

ou seja,

$$
\begin{gathered}
-q\left(\chi_{1} * \chi_{2}\right)^{-}=-\chi_{1}(-1) \chi_{2}(-1) S\left(\chi_{1}\right) S\left(\chi_{2}\right)\left(\chi_{1} \chi_{2}\right)^{-1}(-1) S\left(\left(\chi_{1} \chi_{2}\right)^{-1}\right) \chi_{1}^{-1} \chi_{2}^{-1}= \\
=-\frac{q S\left(\chi_{1}\right) S\left(\chi_{2}\right)}{S\left(\chi_{1} \chi_{2}\right)} \chi_{1}^{-1} \chi_{2}^{-1} .
\end{gathered}
$$

Avaliando em -1 , obtemos o resultado. 
3.1.8. Proposição. $S\left(\curlywedge^{\prime}\right)=S(\curlywedge)$.

Demonstração: A aplicação $x \longmapsto x^{p}$ é um automorfismo de $\mathbb{F}_{q}$, logo $\operatorname{Tr}\left(x^{p}\right)=\operatorname{Tr}(x)$. é,

Seja $m$ um inteiro positivo dividindo $q-1$, e suponhamos que $\backslash$ tenha ordem $m$, isto

Então os valores de \ estão em $\mathbb{Q}\left(\mu_{m}\right)$ e

$$
S(\chi)=S(\chi, \lambda) \in \mathbb{Q}\left(\mu_{m}, \mu_{p}\right) .
$$

Para cada inteiro $c$, primo com $m$, temos o automorfismo $\sigma_{c, 1}$ de $\mathbb{Q}\left(\mu_{m}, \mu_{p}\right)$ tal que

$$
\begin{aligned}
& \sigma_{c, 1}: \zeta_{m} \longrightarrow \zeta_{m}^{c} \\
& \sigma_{c, 1}=1_{Q}\left(\mu_{p}\right)
\end{aligned}
$$

e para cada inteiro $\nu$ primo com $p$, obtemos o automorfismo $\sigma_{1, \nu}$ tal que

$$
\begin{aligned}
& \sigma_{1, \nu}: \zeta_{p} \longrightarrow \zeta_{p}^{\nu} \\
& \sigma_{1, \nu}=1_{q\left(\zeta_{m}\right)}
\end{aligned}
$$

No que segue, $\nu$ é escolhido numa classe de restos $\bmod p$ de tal modo que $\nu$ seja também relativamente primo com $m$. Então:

3.1.9. Proposição. $\sigma_{c, 1} S(\chi)=S\left(\chi^{c}\right)$ e $\sigma_{1, \nu} S(\chi)=\bar{\chi}(\nu) S(\chi)$.

Demonstração: A primeira igualdade é clara, decorrendo da definição. A segunda decorre da mudança de variável

e da observação $\sigma_{1, c} \lambda(x)=\zeta_{p}^{\nu \operatorname{Tr}(x)}=\zeta_{p}^{x \longrightarrow \nu^{-1} x}=\lambda(\nu x)$.

3.1.10. Teorema. Suponhamos que \ tenha ordem m. Então

(a). $S(\chi)^{m} \in \mathbb{Q}\left(\mu_{m}\right)$.

(b). Se b é um inteiro primo com $m$ e $\sigma_{b}=\sigma_{b, 1}$ então $S(\chi)^{b-\sigma_{b}} \in \mathbb{Q}\left(\mu_{m}\right)$.

Demonstração: Para provar (a), basta mostrar que $S(\chi)^{m}$ fica fixo pelos automorfismos $\sigma_{1, \nu}$. Mas $\sigma_{1, \nu} S(\chi)^{m}=\bar{\chi}(\nu)^{m} S(\chi)^{m}$, pois $\chi^{m}=1$. Para provar (b):

Mas

$$
\sigma_{1, \nu} \frac{S(\chi)^{b}}{S(\chi)^{\sigma_{b}}}=\frac{\sigma_{1, \nu} S(\chi)^{b}}{\sigma_{1, \nu} S(\chi)^{\sigma_{b}}} .
$$

$$
S(\chi)^{\sigma_{b}}=\sum_{x \in \mathbb{F}} \chi(x)^{b} \lambda(x)=S\left(\chi^{b}\right)
$$

e portanto,

$$
\sigma_{1, \nu} \frac{S(\chi)^{b}}{S\left(\chi^{b}\right)}=\frac{\bar{\chi}(\nu)^{b} S(\chi)^{b}}{\bar{\chi}(\nu)^{b} S\left(\lambda^{b}\right)}=S(\chi)^{b-\sigma_{b}} .
$$




\subsection{Teoremas de Stickelberger e Kummer}

Nesta seção determinaremos a fatorização em ideais primos das somas de Gauss. Inicialmente será analisado o caso $q=p$. As propriedades que serão utilizadas são:

$$
S(\chi) \in \mathbb{Q}\left(\zeta_{p m}\right), \quad S(\chi)^{\sigma_{1, \mu}-1}=\overline{\chi(v)}, \quad|S(\chi)|=p^{1 / 2} .
$$

É necessário compreender a fatorização do primo $p$ no corpo $\mathbb{Q}\left(\zeta_{p m}\right)$. O primo $p$ se ramifica totalmente em $\mathbb{Q}\left(\zeta_{p}\right)$ e, de fato,

$$
p \sim\left(1-\zeta_{p}\right)^{p-1}
$$

onde $\sim$ denota igualdade a menos da multiplicação por uma unidade e $p$ se decompõe totalmente em $\mathbb{Q}\left(\zeta_{m}\right)$, se $p \equiv 1(\bmod m) \cdot{ }^{[10]}$ Esses dois fatos determinam completamente a ramificação de $p$ em $\mathbb{Q}\left(\zeta_{p m}\right)$.

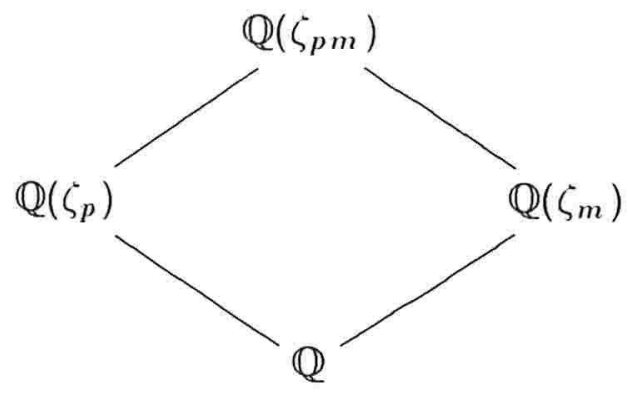

Em $\mathbb{Q}\left(\zeta_{p m}\right)$, o ideal $(p)$ se decompõe totalmente em $\mathbb{Q}\left(\zeta_{m}\right)$ e cada primo que aí aparece se ramifica totalmente em $\mathbb{Q}\left(\zeta_{p m}\right)$.

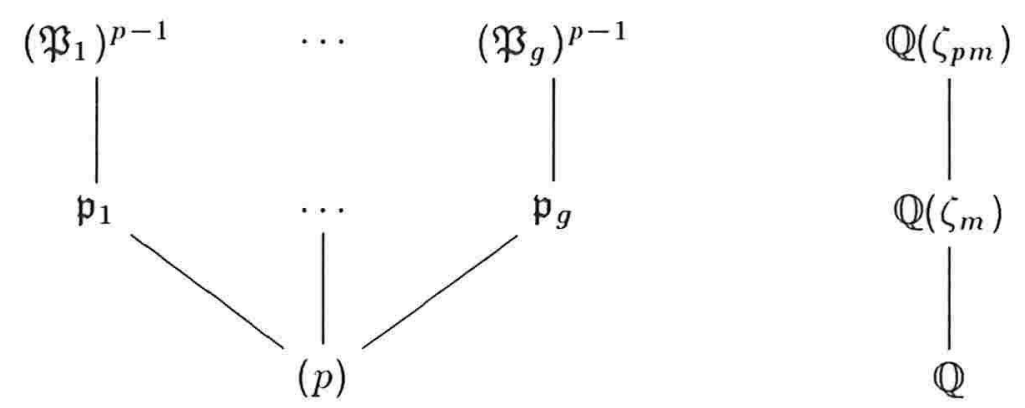

onde $g=\varphi(m)$.

Assim, para cada primo $\mathfrak{P}_{\mathrm{i}}$ acima de $(p)$, o corpo de restos é isomorfo a $\mathbb{F}_{p}$ e o grupo de inércia de $\mathfrak{P}_{i}$ na extensão $\mathbb{Q}\left(\zeta_{p m}\right) / \mathbb{Q}$ é exatamente $\operatorname{Gal}\left(\mathbb{Q}\left(\zeta_{p m}\right) / \mathbb{Q}\left(\zeta_{m}\right)\right)$. De fato, um primo $p \in \mathbb{Z}$ é ramificado numa extensão $\mathbb{L} / \mathbb{Q}$ se e somente se $p$ divide o discriminante de $\mathbb{L} / \mathbb{Q}^{[11]}$, e do fato de $\mathbb{Q}\left(\zeta_{p m}\right)$ ser o compositum dos corpos $\mathbb{Q}\left(\zeta_{p}\right)$ e $\mathbb{Q}\left(\zeta_{m}\right)$, temos que em

[10] Cf. Capítulo 1, teorema 1.2.55.

[11] Cf. teorema 1.2.47. 
$\mathbb{Q}\left(\zeta_{m}\right), p$ não ramifica e como $p \equiv 1(\bmod m)$, pela lei de decomposição ${ }^{[12]}$, temos que $\operatorname{Gal}\left(\mathbb{Q}\left(\zeta_{p m}\right) / \mathbb{Q}\left(\zeta_{m}\right)\right) \cong \operatorname{Gal}\left(\mathbb{Q}\left(\zeta_{p}\right) / \mathbb{Q}\right)$.

O conjunto dos $\hat{\gamma}(m)$ primos $p_{i}$ acima de $(p)$ em $\mathbb{Q}\left(\zeta_{m}\right)$ está em bijeção canônica com o conjunto dos monomorfismos de $\mu_{m}$ em $\mathbb{F}_{p}^{*}$. De fato, ao primo $p$ corresponde a projeção natural

$$
\mu_{m} \stackrel{\pi}{\longrightarrow} \mathbb{Z}\left[\zeta_{m}\right] / p
$$

que é um monomorfismo, pois $x^{m}-1$ é separável $(\bmod p)$.

Como $\mathbb{Z}\left[\zeta_{m}\right] / \mathfrak{p}_{i} \cong \mathbb{F}_{p}$, para cada $1 \leq i \leq \varphi(m)$, a cada primo $\mathfrak{p}$ associemos o monomorfismo obtido da composta dos dois anteriores

$$
\mu_{m} \stackrel{\pi_{\mathfrak{p}}}{\longrightarrow} \mathbb{F}_{p}^{*}
$$

Isso quer dizer que, fixado um primo $\mathfrak{p}$ em $\mathbb{Q}\left(\zeta_{m}\right)$ sobre $(p)$, existe um único caracter $\omega$ de $\mathbb{F}_{p}^{*}$ tal que

$$
\omega(u) \equiv u(\bmod \mathfrak{p}) .
$$

Tal caracter é chamado o caracter de Teichmuller e é um gerador do grupo de caracteres de $\mathbb{F}_{p}^{*}$ de ordem $m$.

Como na seção 1, é possível escolher o seguinte caracter de Teichmuller: escolhemos uma raiz primitiva $g(\bmod p)$, então $\backslash(g)$ é um gerador de $\mu_{m}$ e $g^{(p-1) / m}$ é um elemento de ordem $m$ em $\mathbb{F}_{p}^{*}$ considere $\omega$ que leva $\chi(g)$ em $g^{(p-1) / m}$, seja p o ideal primo de $\mathbb{Q}\left(\zeta_{m}\right)$ acima de $p^{[13]}$ correspondente a $\omega$ e seja $\mathfrak{P} \circ$ primo de $\mathbb{Q}\left(\zeta_{p m}\right)$ acima de $\mathfrak{p}$ então $\mathfrak{P}^{p-1}=\mathfrak{p}$.

Em termos de congruências temos

$$
\curlyvee(x) \equiv x^{(p-1) / m}(\bmod \mathfrak{P}) \quad \forall x \in \mathbb{F}_{p}^{*} .
$$

Seja $v_{\mathfrak{p}}$ a valorização de $\mathbb{Q}\left(\zeta_{p m}\right)$ correspondente ao ideal $\mathfrak{P}$ então $v_{\mathfrak{P}}(p)=p-1$, e portanto $v_{\mathfrak{p}}\left(1-\zeta_{p}\right)=1$, ou seja, $\left(1-\zeta_{p}\right)$ é um uniformizante local ${ }^{[14]}$ para $\mathfrak{P}$.

Para cada inteiro $0<c<m$ e primo $\operatorname{com} m$, definindo o inteiro $k_{c}$ por

$$
k_{c}=v_{\mathfrak{p}}\left(S(\uparrow)^{\sigma_{c, 1}}\right) .
$$

Isto é, $k_{c}$ é o expoente de $\mathfrak{P}$ na fatorização de $S(\backslash)^{\sigma_{c, 1}} \mathrm{em} \mathbb{Q}\left(\zeta_{p m}\right)$. É também o expoente de $\mathfrak{P}^{\sigma_{c, 1}^{-1}}$ na fatorização de $S(\chi)$, visto que o grupo de Galois age transitivamente nos primos acima de $(p)^{[15]}$. O elemento de Stickelberger será definido por

$$
\theta=\sum_{c} \frac{c}{m} \sigma_{c, 1}^{-1}
$$

[12] Cf. Capítulo 1, teorema 1.2.55.

[13] Cf. Capítulo 1, teoremas $1.2 .55,1.2 .53,1.2 .51$ e 1.2.47.

[14] Cf. Capítulo 1, observação 1.2.28.

[15] Cf. Capítulo 1, teorema 1.2.21. 
onde a soma é feita sobre todos os inteiros $0<c<m$ primos com $m$. É um elemento do anel de grupo $\mathbb{Q}\left[\operatorname{Gal}\left(\mathbb{Q}\left(\zeta_{m}\right) / \mathbb{Q}\right)\right]$, onde $\zeta_{m}$ é uma raiz primitiva $m$-ésima da unidade.

Se $\mathfrak{a}$ for um ideal fracionário em $\mathbb{Q}\left(\zeta_{m}\right)$ e $a \in \mathbb{Q}\left[\operatorname{Gal}\left(\mathbb{Q}\left(\zeta_{m}\right) / \mathbb{Q}\right)\right]$ então, se o ideal fracionário gerado por $\mathfrak{a}$ em $I\left(\mathbb{Q}\left(\zeta_{p m}\right)\right)$ for $\mathfrak{b}^{r}$, para um ideal fracionário $\mathfrak{b}$ em $I\left(\mathbb{Q}\left(\zeta_{p m}\right)\right)$ e $r \in \mathbb{Z} \operatorname{com} r a \in \mathbb{Z}\left[\operatorname{Gal}\left(\mathbb{Q}\left(\zeta_{m}\right) / \mathbb{Q}\right)\right]$, convencionamos que

$$
\mathfrak{a}^{\alpha}:=\mathfrak{b}^{r \alpha} .
$$

Assim, é claro que $\mathfrak{p}^{\theta}=\mathfrak{P}^{(p-1) \theta}$.

3.2.1. Teorema (Stickelberger). Na situação acima, $(S(\chi))=\mathfrak{P}^{(p-1) \theta}$, onde $(S(\chi))$ é o ideal gerado por $S(\chi)$.

$\mathrm{Na}$ prova desse teorema, será utilizado um lema bastante simples, cuja formulação geral é: Seja $\mathbb{F}$ um corpo, v uma valorização discreta em $\mathbb{F}$ com anel de valorização $\mathcal{O}_{v}$ e ideal maximal $P_{v}$. Seja $F_{v}=\mathcal{O}_{v} / P_{v}$ o corpo de restos de $v$ e $\pi \in \mathbb{F}$ um uniformizante de $v,{ }^{[16]}$ isto é, tal que $v(\pi)=1$.

Usaremos o homomorfismo

$$
\lambda: \mathbb{F}^{*} \longrightarrow \mathbb{Z} \times \mathbb{F}_{v}^{*}
$$

definido por

$$
\alpha \in \mathbb{F}^{*} \longmapsto\left(v(\alpha), \alpha / \pi^{v(\alpha)}\right) \in \mathbb{Z} \times \mathbb{F}_{v}^{*} .
$$

Chama-se o grupo de inércia de $I_{v}$ de $\mathbb{F}$ em $v$ o grupo daqueles automorfismos de $\mathbb{F}$ que fixam $v$ e induzem $o$ automorfismo trivial no corpo de restos de $v, \mathbb{F}_{v}$. Seja $\gamma \in I_{v}$ um elemento dado. Considere o homomorfismo $\rho$ de $\mathbb{F}^{*}$ em si mesmo tal que $\rho(\alpha)=\gamma(\alpha) / \alpha$. São imediatas as seguintes propriedades:

(1). Se $u \in \mathbb{F}$ é tal que $v(u)=0$ então $\lambda(\rho(u))=(0,1)$.

(2). $\lambda(\rho(\pi))=(0, z)$ onde $z$ é a classe de restos de $\gamma(\pi) / \pi$.

3.2.2. Lema. Seja $\alpha \in \mathbb{F}^{*}$. Se $\lambda(\alpha)=(k, r)$, então $\lambda(\rho(\alpha))=\left(0, z^{k}\right)$.

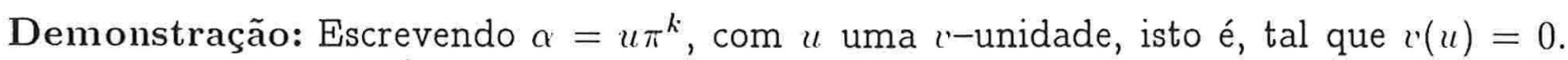
Então $\rho(\alpha)=\rho(u) \rho(\pi)^{k}$. Como $\lambda$ é um homomorfismo de grupos e valem (1) e (2) acima, prova-se o lema.

[16] Cf. Capítulo 1, observação 1.2.28. 
Observação. O lema foi feito para o seguinte tipo de aplicação: dado a $\in \mathbb{F}^{*}$. queremos calcular r(a). Tomemos um elemento năo trivial $; \in I_{r}$ e calculemos $\epsilon_{0} \in \mathbb{N} \cup\{\infty\}$ como a ordem de $\gamma(\pi) / \pi$ em $\mathbb{F}_{r}^{*}$. Feito isso. procuremos um inteiro $k$ tal que

$$
\gamma_{i}(\alpha) / \alpha \equiv(\gamma(\pi) / \pi)^{k}\left(\bmod P_{r}\right) .
$$

Segue-se que $v(\alpha) \equiv k\left(\bmod \epsilon_{0}\right)\left(\right.$ se $\epsilon_{0}=\infty$, obtemos simplesmente a igualdade).

Demonstração do teorema de Stickelberger: Notemos que $S(\backslash)$ é um inteiro algébrico de $\mathbb{Q}\left(\zeta_{p m}\right)$ que divide $p$. Logo, apenas os primos que estão acima de $p$ podem aparecer na fatorização de $S(\chi)$.

Tomemos $0<c<m$ primo com $m$ e calculemos $k_{c}=v_{\mathfrak{P}}\left(S(\Upsilon)^{\sigma_{c, 1}}\right)$. Aplicando o lema na situação: $\mathbb{F}=\mathbb{Q}\left(\zeta_{p m}\right), v=v_{\mathfrak{p}}, \alpha=S(\chi)^{\sigma_{c, 1}}, \pi=1-\zeta_{p}$ e $\gamma=\sigma_{1 . g}$, onde $g$ é uma raiz primitiva mod $p$, isto é, um gerador de $\mathbb{F}_{p}^{*}$. Neste caso, $k=k_{c}$ e o corpo de restos $\mathbb{F}_{v}=\mathbb{F}_{p}$.

A escolha satisfaz as hipóteses do lema, pois $\sigma_{1 . g} \in \operatorname{Gal}\left(\mathbb{Q}\left(\zeta_{p}\right) / \mathbb{Q}\right)$, que é o grupo de inércia de $\mathfrak{P}$ na extensão $\mathbb{Q}\left(\zeta_{p m}\right) / \mathbb{Q}$.

Logo

$$
\rho(\alpha)=S(\chi)^{\sigma_{c, 1}\left(\sigma_{1, g}-1\right)}=S\left(\chi^{c}\right)^{\sigma_{1, g}-1}=\bar{\chi}^{c}(g) .
$$

Como $\bar{\Upsilon}^{c}(g)=\bar{\Upsilon}^{c}(g) \equiv g^{(p-1) c / m}(\bmod \mathfrak{P})$, temos

$$
\lambda(\rho(\alpha))=\left(0, g^{(p-1) c / m}\right) .
$$

Por outro lado,

$$
z=\left(1-\zeta_{p}\right)^{\sigma_{1, g}-1}=\frac{1-\zeta_{p}^{g}}{1-\zeta_{p}}=\sum_{i=0}^{g-1} \zeta_{p}^{i} \equiv\left(\sum_{i=0}^{g-1} \zeta_{p}^{i}\right)^{p}(\bmod \mathfrak{P}) .
$$

Como esta última expressão é congruente a $g$ módulo $\mathfrak{P}$, segue

$$
\left(0, z^{k}\right)=\left(0, g^{k_{c}}\right), \quad g^{(p-1) c / m} \equiv g^{k_{c}}(\bmod p) .
$$

Pela escolha de $g$,

$$
k_{c} \equiv \frac{(p-1) c}{m}(\bmod (p-1)) .
$$

Como $0<(p-1) c / m<p-1$ e $0 \leq k_{c} \leq v_{\mathfrak{p}}(p)=p-1$, temos, de fato, a igualdade

$$
k_{c}=\frac{(p-1) c}{m} .
$$

A próxima definição servirá para obtermos explicitamente elementos primitivos de corpos ciclotômicos de determinados graus. 
3.2.3. Definição. Sejam $p$ um primo positivo, g uma raiz primitiva de $\mathbb{Z}_{p}$ e $\zeta_{p}$ uma raiz primitiva $p$-ésima da unidade. Definimos os períodos gaussianos, ou períodos ciclotômicos gaussianos de $\mathbb{Q}\left(\zeta_{p}\right) / \mathbb{Q}$ como sendo os seguintes números

$$
\eta_{i, \epsilon}=\sum_{j=0}^{\frac{p-3}{2}} \zeta_{p}^{\epsilon j+i}, \quad 0 \leq i \leq(\epsilon-1)
$$

onde $e$ percorre todos os divisores de $p-1$.

3.2.4. Teorema (Kummer). Seja $m$ um inteiro positivo arbitrário, $p$ um primo racional com $p \equiv 1(\bmod m), \mathbb{L} / \mathbb{Q}$ o subcorpo cíclico de grau $m$ dentro de $\mathbb{Q}\left(\zeta_{p}\right), \eta$ um período ciclotômico gaussiano gerando $\mathbb{L}$ sobre $\mathbb{Q}$ e $\nmid$ um caracter de $G:=\operatorname{Gal}(\mathbb{L} / \mathbb{Q})$ de ordem $m$. Então:

(a). $S(\backslash)=\sum_{\sigma \in G} \backslash(\sigma) \sigma(\eta)$.

(b). $S(\curlyvee)^{m} \in \mathbb{Q}\left(\zeta_{m}\right)$ e $\mathbb{L}\left(\zeta_{m}\right)=\mathbb{Q}\left(\zeta_{m}, S(\chi)\right)$.

(c). A decomposição de $S(\chi)^{m}$ em $\mathbb{Q}\left(\zeta_{p m}\right)$ é dada por

$$
\left(S(\chi)^{m}\right)=\Delta(\mathfrak{p})
$$

onde $\mathfrak{p}$ é um ideal primo acima de $p$ em $\mathbb{Q}\left(\zeta_{m}\right)$ convenientemente escolhido e

$$
\Delta=\sum_{\sigma \in G} r\left(\sigma^{-1}\right) \sigma .
$$

Demonstração: Os subcorpos de $\mathbb{Q}\left(\zeta_{p}\right)$ podem ser determinados explicitamente pelos chamados períodos gaussianos, do seguinte modo: seja $g$ uma raiz primitiva $(\bmod p)$ e $\epsilon$ um divisor fixo de $p-1$. Seja $f=(p-1) / e$ e tomemos os períodos

$$
\eta_{i, e}=\sum_{j=0}^{f-1} \zeta_{p}^{e j+i}, \quad 0 \leq i \leq(e-1) .
$$

Seja $\sigma \circ$ automorfismo de $\mathbb{Q}\left(\zeta_{p}\right)$ dado por $\zeta_{p} \longmapsto \zeta_{p}^{g}$. Como g é raiz primitiva, $\sigma$ gera $\circ$ grupo de Galois de $\mathbb{Q}\left(\zeta_{p}\right)$ sobre $\mathbb{Q}$. O subgrupo de ordem $f$ é

$$
H=\left\{1, \sigma^{e}, \ldots, \sigma^{\epsilon(f+1)}\right\},
$$

correspondendo ao subgrupo

$$
\left\{1, g^{e}, \ldots, g^{e(f-1)}\right\} \subseteq(\mathbb{Z} / p \mathbb{Z})^{*} .
$$

Conseqüentemente, $\left\{g^{e f+i} \mid 0 \leq j \leq(f-1)\right\}$ é uma coclasse desse subgrupo. É fácil ver que $H$ fixa $\eta_{i, e}$ e que $\sigma\left(\eta_{i, e}\right)=\eta_{i+1, e}$ para $0 \leq i \leq(\epsilon-2)$ e $\sigma\left(\eta_{e-1, \epsilon}\right)=0$. Assim, $\eta_{i, \epsilon}$ possui 
exatamente $\epsilon$ conjugados sob $\operatorname{Gal}\left(\mathbb{Q}\left(\zeta_{p}\right) / \mathbb{Q}\right)$, de onde decorre que $\eta_{\text {i.t }}$ gera o subgrupo de $\mathbb{Q}\left(\zeta_{p}\right)$, de grau $\epsilon$, sobre $\mathbb{Q}$.

Por outro lado, o caracter $\lambda: \mathbb{F}_{p} \longrightarrow \mathbb{C}^{*}$, dado pelo traço induz a forma bilinear

$$
(x . y) \longmapsto \lambda(x y)
$$

e isso prova (a). A primeira parte do item (b) já foi provada. Para a segunda afirmação do item (b), basta notar que $S(\uparrow)$ é o resolvente de Lagrange associado à extensão de Kummer $\mathbb{Q}\left(\zeta_{m}, \eta\right) / \mathbb{Q}\left(\zeta_{m}\right)$. Para provar (c), usamos o teorema de Stickelberger, que fornece a decomposição de $S(\chi)$ em $\mathbb{Q}\left(\zeta_{p m}\right)$.

$$
(S(\backslash))=\mathfrak{P}^{(p-1) \theta} .
$$

Elevando a $m$,

$$
(S(\chi))^{m}=\left(S(\chi)^{m}\right)=\mathfrak{P}^{m(p-1) \theta}
$$

e portanto, abaixando para o corpo $\mathbb{Q}\left(\zeta_{m}\right)$, temos

$$
\left(S(\lambda)^{m}\right)=\mathfrak{p}^{m \theta}=\Delta(\mathfrak{p}) .
$$

O próximo teorema será usado para demonstrar que uma extensão cíclica de $\mathbb{Q}$, de grau $\ell^{n}$, onde $\ell$ é um primo, tem como elemento primitivo, o produto de uma raiz $m$-ésima da unidade, um período ciclotômico e um primo. Isso será importante para o teorema 4.1.2, resultado que mostra que uma extensão cíclica de $\mathbb{Q}^{\prime}$ de grau $m$, cujo elemento primitivo pode ser tomado da forma $\Delta(\gamma)$, é um corpo ciclotômico.

3.2.5. Teorema (Weber). Sejam $\left(\right.$ um primo, $m=\ell^{n}$ e $n \geq 2$ para $\ell=2$, $p$ um primo racional com $p \equiv 1(\bmod \ell)$ para $\ell \neq 2$ ou $p \equiv 1(\bmod 4)$ para $\ell=2$. Sejam $m_{1}=\operatorname{mdc}(m, p-1), m_{2}:=m \cdot m_{1}^{-1}, \mathfrak{p}$ um ideal primo que divide $(p)$ em $\mathbb{Q}\left(\zeta_{m}\right)$ e $\eta$ algum período ciclotômico em $\mathbb{Q}\left(\zeta_{p}\right)$, que gera o subcorpo cíclico de grau $m_{1}$ em $\mathbb{Q}\left(\zeta_{p}\right)$. Seja

$$
\left(\zeta_{m}, \eta\right)=\sum_{\sigma} \backslash(\sigma) \sigma(\eta)
$$

uma soma de Gauss onde $\sigma$ percorre todo o grupo $\operatorname{Gal}(\mathbb{Q}(\eta) / \mathbb{Q})$ e \é um caracter primitivo deste grupo. Então

$$
\Delta(\mathfrak{p})=\left(p^{\frac{m\left(m_{2}-1\right)}{2}} \cdot\left(\zeta_{m_{1}}, \eta\right)^{m}\right) .
$$

Demonstração: v. [WEBER, 22] 


\subsection{Um resultado parcial}

Nesta seção, apresentamos mais alguns resultados sobre corpos ciclotômicos, bem como um caso particular do teorema de Kronecker-Weber, o caso em que a extensão $\mathbb{L} / \mathbb{Q}$ é totalmente real e cujo grau de $\mathbb{L}$ sobre $\mathbb{Q}$ é uma potência de 2 , maior ou igual a 4. Estudaremos o caso em que o grau de $\mathbb{L} / \mathbb{Q}$ for 2 , no capítulo 4 , quando tratarmos especificamente do caso quadrático.

3.3.1. Lema. Seja a um inteiro algébrico cujos conjugados tem valor absoluto 1. Então $\alpha$ é uma raiz da unidade.

Demonstração: Sejam $p_{1}, p_{2}, \ldots, p_{m}, \ldots$ os polinômios irredutíveis de $\alpha, \alpha^{2}, \ldots \alpha^{m}, \ldots$, respectivamente.

Para cada $i \in \mathbb{N}$ o grau de $p_{i}$ é menor ou igual ao grau de $p_{1}$, para todo $i \geq 1$ e cada raiz tem valor absoluto 1 .

De fato, $\alpha$ é um inteiro algébrico, portanto $\alpha^{m}$ é elemento de $\mathbb{Z}[\alpha] \subset \mathbb{Q}(\alpha)$. Como $\alpha^{m}$ é um número algébrico, tal que $\mathbb{Q}\left(a^{m}\right) \subset \mathbb{Q}(\alpha)$, temos que para todo $i \in \mathbb{N}, p_{i}(\alpha)$ tem grau menor ou igual ao grau de $p_{1}$ e do fato dos conjugados de $\alpha$ terem valor absoluto 1 , temos:

$$
|\beta|=1 \Rightarrow\left|\beta^{j}\right|=|\beta|^{j}=1^{j}=1
$$

para todo 3 conjugado de $\alpha$ e qualquer $j \geq 1$.

Fixemos $i \in \mathbb{N}$. Os coeficientes de $p_{i}$ são funções simétricas das raízes e como cada raiz de $p_{i}$ tem valor absoluto 1 e o grau de $p_{i}$ é limitado, temos que os coeficientes de $p_{i}$ são inteiros racionais limitados, dependendo somente do grau de $\alpha$ sobre $\mathbb{Q}$.

Portanto existe apenas uma quantidade finita de polinômios irredutíveis que tem uma potência de $\alpha$ como raiz, ou seja, existem $n, k \geq 1$, tais que $\alpha^{k}=\alpha^{n+k}$, $\log \alpha^{n}=1$.

3.3.2. Lema. Sejam $\left(\right.$ um primo arbitrário, $m=\ell^{n}$ com $m \geq 3, \zeta_{m}$ uma raiz $m$-ésima primitiva da unidade, $\mathbb{Q}^{\prime}=\mathbb{Q}\left(\zeta_{m}\right)$ e $\mathbb{Q}^{\prime+}:=\mathbb{Q}\left(\zeta_{m}+\zeta_{m}^{-1}\right)$. Então toda unidade em $\mathbb{Q}^{\prime}$ é um produto de uma raiz m-ésima da unidade com alguma unidade em $\mathbb{Q}^{\prime+}$.

Demonstração: Notemos que

$$
\left[\mathbb{Q}^{\prime}: \mathbb{Q}^{\prime+}\right]=2
$$

e portanto $\operatorname{Gal}\left(\mathbb{Q}^{\prime} / \mathbb{Q}^{\prime+}\right)$ é formado pela identidade em $\mathbb{Q}^{\prime}$ e pela conjugação complexa.

Sejam $\Sigma$ uma unidade de $\mathbb{Q}^{\prime}$ e $\sigma \in \operatorname{Gal}\left(\mathbb{Q}^{\prime} / \mathbb{Q}^{\prime+}\right)$ a conjugação complexa. Então $\frac{\sigma(\varepsilon)}{\varepsilon}$ é outra unidade, e

$$
N_{Q^{\prime} / Q^{\prime}}+\left(\frac{\sigma(\varepsilon)}{\xi}\right)=1
$$

Como $\frac{\sigma(\varepsilon)}{\varepsilon} \in \mathbb{Q}^{\prime}$ e $\sigma$ é a conjugação complexa, temos que $\left|\frac{\sigma(\varepsilon)}{\varepsilon}\right|=1$. Portanto, pelo lema anterior, $\frac{\sigma(\varepsilon)}{\varepsilon}= \pm \zeta_{m}^{k}$ onde $k \leq m$. 
Nosso primeiro objetivo é mostrar que $\frac{\sigma(\varepsilon)}{\varepsilon}$ é um quadrado em $\mathbb{Q}^{\prime}$. Há dois casos a considerar, quando $C=2$ e quando $C \neq 2$.

No caso $t \neq 2, m=l^{n}$ é ímpar, de onde decorre que 2 é inversível módulo $m$ e portanto $\zeta_{m}^{k}$ é um quadrado, que será indicado por $\zeta_{m}^{2 t}$.

Suponhamos, por absurdo, que $\frac{\sigma(\varepsilon)}{\tilde{\varepsilon}}=-\zeta_{m}^{2 t}$. Seja $\tau$ a projeção canônica de $\mathcal{O}_{\mathbb{L}}$ em $\mathcal{O}_{\mathbb{L}} /\left(1-\zeta_{m}\right)$, onde $\left(1-\zeta_{m}\right)$ denota o ideal principal gerado por $1-\zeta_{m}$.

Notemos que $\zeta_{m}$ é uma unidade em $\mathbb{Q}^{\prime}$ e $\tau\left(\zeta_{m}\right)=\overline{1}$ em $\mathcal{O}_{\mathbb{L}} /\left(1-\zeta_{m}\right)$, temos também que $\tau\left(\sigma\left(\zeta_{m}\right)\right)=\tau\left(\zeta_{m}^{m-1}\right)=\overline{1}$ e $\tau(1)=\overline{1}$, onde $\bar{\alpha}$ representa a classe de $\alpha$. A projeção de um elemento da forma

$$
\sum_{j=1}^{n} a_{j} \zeta_{m}^{j}
$$

onde $a_{j} \in \mathbb{Q}$ coincide com a projeção de

$$
\sum_{j=1}^{n} \sigma\left(a_{j} \zeta_{m}^{j}\right)
$$

pois

$$
\sum_{j=1}^{n} \tau\left(\sigma\left(a_{j} \zeta_{m}^{j}\right)\right)=\sum_{j=1}^{n} \tau\left(a_{j} \sigma\left(\zeta_{m}^{j}\right)\right)=\sum_{j=1}^{n} \tau\left(a_{j}\right) \tau\left(\zeta_{m}^{-j}\right)=\sum_{j=1}^{n} \tau\left(a_{j}\right)
$$

onde $n=\varphi(m)$ e $a_{j}^{\prime} s \in \mathbb{Q}$.

Se $\sigma(\varepsilon)=-\zeta_{m}^{k} \varepsilon$, teríamos $\tau(\varepsilon)=\tau(\sigma(\varepsilon))=-\tau(\varepsilon)$. Assim

$$
\tau(\varepsilon)=-\tau(\varepsilon) \Longleftrightarrow \tau(2 \varepsilon)=2 \tau(\varepsilon)=0 \Longleftrightarrow 2 \varepsilon \in\left(1-\zeta_{m}\right) .
$$

Como é uma unidade, então $2 \in\left(1-\zeta_{m}\right) \cap \mathbb{Z} \subseteq C \mathbb{Z}$, absurdo, pois $\ell$ é um primo diferente de 2.

No caso $\ell=2$, temos que $\sigma(\varepsilon)=\zeta_{m}^{a} \varepsilon \operatorname{com} a$ sendo par (não foi necessário supor que $\sigma(\varepsilon)= \pm \zeta_{m}^{a} \varepsilon$, porque no caso $(=2,-1$ é um quadrado).

Suponhamos, por absurdo, que $\sigma(\varepsilon)=\zeta_{m}^{a}=$ com a ímpar, podemos supor, sem perda de generalidade, que a é 1 , pois caso contrário, podemos fazer essas contas com $\zeta_{\frac{a-1}{2}} \varepsilon$, $\circ$ que não altera o nosso resultado. Então o ideal diferente de $\varepsilon$ em relação à extensão $\mathbb{Q}^{\prime} / \mathbb{Q}^{\prime}$ é simplesmente $(\varepsilon-\sigma(\varepsilon))=\left(1-\zeta_{m}^{a}\right)=\left(1-\zeta_{m}\right)$, pois tanto $\varepsilon$, como $\zeta_{m}$ são unidades.

Assim, temos, pelo teorema do divisor, ${ }^{[1 \tau]}$ que o ideal diferente de $\mathbb{Q}^{\prime} / \mathbb{Q}^{\prime+}$ divide o ideal diferente de $\varepsilon$. Mas o ideal diferente de $\mathbb{Q}^{\prime} / \mathbb{Q}^{\prime+}$ é o ideal

$$
\left(\zeta_{m}-\sigma\left(\zeta_{m}\right)\right)=\left(\zeta_{m}-\zeta_{m}^{-1}\right)=\left(1-\zeta_{m}^{2}\right)=\left(1-\zeta_{m}\right)^{2}
$$

[1i] Cf. Capítulo 1, teorema 1.2.42. 
Como $\left(1-\zeta_{m}\right)$ é um ideal primo, se a for ímpar temos um absurdo!

Portanto $\sigma(\varepsilon)=\varepsilon \zeta_{m}^{-2 t}$ para algum $t<m$. Logo $\sigma\left(\Sigma \zeta_{m}^{t}\right)=\Sigma \zeta_{m}^{-2 t} \zeta_{m}^{-t}=\Sigma \zeta_{m}^{-t}$, isto é, $\Sigma \zeta_{m}^{-t}$ é uma unidade em $\mathbb{Q}^{\prime+} \mathrm{e}$

$$
\varepsilon=\left(\Sigma \zeta_{m}^{-t}\right) \zeta_{m}^{-t} \text {. }
$$

3.3.3. Lema. Seja $\mathbb{L} / \mathbb{Q}$ uma extensão galoisiana, cujo grau é $2^{n}(n \in \mathbb{N})$, totalmente real e que não ramifica fora de 2. Então $\mathbb{L} / \mathbb{Q}$ é ciclotômica e coincide com o corpo

$$
C_{n}:=\mathbb{Q}\left(\zeta_{2^{n+2}}+\zeta_{2^{n+2}}^{-1}\right) \text {. }
$$

Demonstração: Caso $n=0$, então $\mathbb{L}=\mathbb{Q}$ e nada há a fazer.

Caso $n=1$, então $\mathbb{L}=\mathbb{Q}(\sqrt{2})$. Isso decorre do fato de $\mathbb{L} / \mathbb{Q}$ ser um corpo quadrático que não ramifica fora de 2. De fato, as únicas extensões quadráticas de grau 2 de $\mathbb{Q}$ que não ramificam fora de 2 são $\mathbb{L}=\mathbb{Q}(\sqrt{2})$ ou $\mathbb{L}=\mathbb{Q}(\sqrt{-2})$, pois a ramificação só ocorre nos primos que dividem o discriminante. ${ }^{[18]}$ Como $\mathbb{L}$ é totalmente real, $\mathbb{L}=\mathbb{Q}(\sqrt{2})$, logo

$$
\mathbb{L} \subseteq \mathbb{Q}(\sqrt{2}, \sqrt{-1}) \cap \mathbb{R}=\mathbb{Q}\left(\zeta_{8}\right) \cap \mathbb{R}=\mathbb{Q}\left(\zeta_{8}+\zeta_{8}^{-1}\right)=\mathbb{Q}\left(\zeta_{2^{n+1}}+\zeta_{2^{n+1}}^{-1}\right)=C_{1}
$$

Caso $n>1$ : Sejam $\sigma$ um gerador de $\operatorname{Gal}\left(C_{n} / \mathbb{Q}\right), \tau$ uma extensão de $\sigma$ a $\operatorname{Gal}\left(\mathbb{L} \cdot C_{n} / \mathbb{Q}\right)$ e $\mathbb{K}=\left(\mathbb{L} \cdot C_{n}\right)^{\langle\tau\rangle}$, o corpo fixo pelo grupo gerado por $\tau$.

Notemos que $\mathbb{K} \cap C_{n}=\mathbb{Q}$, pois $x \in \mathbb{K} \cap C_{n} \Rightarrow \tau(x)=\sigma(x)=x \Rightarrow x \in \mathbb{Q}$, pois $\sigma$ é um gerador de $\operatorname{Gal}\left(C_{n} / \mathbb{Q}\right)$.

Se $[\mathbb{K}: \mathbb{Q}]>1$ então $\mathbb{K} \supset \mathbb{Q}(\sqrt{2})$. De fato, $\mathbb{K}$ estando contido no compositum de corpos totalmente reais e que não ramificam fora de 2 , $\mathbb{K}$ seria totalmente real e não ramificaria fora de 2 , e portanto, sendo os grupos de Galois de $\mathbb{L} / \mathbb{Q}$ e $C_{n} / \mathbb{Q}$ de ordens potências de 2 , teríamos que o grupo de Galois de $\mathbb{K} / \mathbb{Q}$ seria abeliano cuja ordem seria potência de 2 , logo conteria um subcorpo de grau 2, totalmente real que não ramifica fora de 2, a saber $\mathbb{Q}(\sqrt{2})$, o que implicaria que $\mathbb{K} \cap C_{n} \supset \mathbb{Q}(\sqrt{2})$, o que seria um absurdo. Portanto $[\mathbb{K}: \mathbb{Q}]=1$, ou seja, $\mathbb{K}=\mathbb{Q}$.

Notemos que $\tau$ tem ordem $2^{n}$, pois $\operatorname{Gal}\left(\mathbb{L} \cdot C_{n} / \mathbb{Q}\right) \hookrightarrow \operatorname{Gal}(\mathbb{L} / \mathbb{Q}) \times \operatorname{Gal}\left(C_{n} / \mathbb{Q}\right)$ e $\left.\tau\right|_{C_{n}}$ é um gerador de $\operatorname{Gal}\left(C_{n} / \mathbb{Q}\right)$, que tem ordem $2^{n}$.

Como $\mathbb{Q}=\left(\mathbb{L} \cdot C_{n}\right)^{\langle\tau\rangle}$, pelo teorema fundamental da teoria de Galois, temos $\langle\tau\rangle=$ $\operatorname{Gal}\left(\mathbb{L} \cdot C_{n} / \mathbb{Q}\right)$ e do fato de $\left.\tau\right|_{C_{n}}=\sigma$ (gerador de $\left.\operatorname{Gal}\left(C_{n}^{\prime} / \mathbb{Q}\right)\right)$, temos que $\operatorname{Gal}\left(C_{n} / \mathbb{Q}\right)=$ $\operatorname{Gal}\left(\mathbb{L} \cdot C_{n}^{\prime} / \mathbb{Q}\right)$, portanto $\mathbb{L} \cdot C_{n} / \mathbb{Q}$ tem grau $2^{n}$ e como $C_{n}^{\prime} / \mathbb{Q}$ tem grau $2^{n}$ e $C_{n} \subset \mathbb{L} \cdot C_{n}$, temos que $\mathbb{L} \cdot C_{n}=C_{n} \Rightarrow \mathbb{L} \subset C_{n}$.

[18] Cf. Capítulo 1, teorema 1.2.47. Vale notar que o discriminante de um corpo quadrático $\mathbb{Q}(\sqrt{m}), m \in \mathbb{Z}(|m|>1$ livre de quadrados $)$ é $m$ ou $4 m$. Cf. Capítulo 1, teorema 1.2.48. 
3.3.4. Teorema (Weber). Se $L^{+}$é o é o grupo das unidades totalmente positivas de $C_{n}$ e $L^{T}$ é o grupo das unidades de $C_{n}$, então $L^{+}=L^{2}$.

Demonstração: Seja c uma unidade totalmente positiva em $C_{n}$, isto é, tal que para todo monomorfismo $\tau: C_{n} \longrightarrow \bar{C}_{n}$ que fixa $\mathbb{Q}, \tau(\xi) \in \mathbb{R}$ e $\tau(\xi)>0$. Considere $C_{n}(\sqrt{\xi})$ uma extensão de $C_{n}$. então

Suponhamos que $C_{n}(\sqrt{\varepsilon}) / \mathbb{Q}$ seja galoisiana e $C_{n}^{\prime} \neq C_{n}(\sqrt{\varepsilon})$, seja $\sigma \in \operatorname{Gal}\left(C_{n}(\sqrt{\varepsilon}) / \mathbb{Q}\right)$,

$$
\sigma(\sqrt{\varepsilon}) \in C_{n}^{\prime}(\sqrt{\varepsilon}) \text {, }
$$

de onde

$$
\sigma(\sqrt{\Sigma})=a_{\sigma}+b_{\sigma} \sqrt{\varepsilon},
$$

$\operatorname{com} a_{\sigma}, b_{\sigma} \in C_{n}$. Logo

$$
\sigma\left(\sqrt{\varepsilon}^{2}\right)=a_{\sigma}^{2}+\varepsilon b_{\sigma}^{2}+2 a_{\sigma} b_{\sigma} \sqrt{\varepsilon},
$$

que implica, $a_{\sigma} b_{\sigma}=0$. Como $\sigma(\sqrt{\varepsilon}) \notin C_{n}$, concluímos que $b_{\sigma} \neq 0$, de onde, $a_{\sigma}=0$.

Portanto,

$$
\sigma(\sqrt{\varepsilon})=b_{\sigma} \sqrt{\varepsilon} \Rightarrow \sigma(\varepsilon)=b_{\sigma}^{2} \varepsilon \text {. }
$$

Como

$$
\sigma \in \operatorname{Gal}\left(C_{n}(\sqrt{\varepsilon}) / \mathbb{Q}\right),
$$

tomando $k \in \mathbb{N}^{*}$ tal que $k=\left|\operatorname{Gal}\left(C_{n}(\sqrt{\varepsilon}) / \mathbb{Q}\right)\right|$ temos $\left.\sigma^{k}\right|_{C_{n}}=1_{C_{n}}, \log$ o

$$
\varepsilon=\sigma^{k}(\varepsilon)=\left(b_{\sigma}^{2}\right)^{k} \varepsilon \Rightarrow b_{\sigma}^{2 k}=1 \text {. }
$$

Como $\varepsilon$ é uma unidade totalmente positiva, $\sigma(\varepsilon)$ também é totalmente positiva, de onde

$$
b_{\sigma}=1
$$

Logo $C_{n}(\sqrt{\tilde{\varepsilon}})=C_{n}^{\prime}$, pois ह é fixo por $\operatorname{Gal}\left(C_{n}(\sqrt{\varepsilon}) / \mathbb{Q}\right)$, o que é uma contradição.

Se $C_{n}(\sqrt{\varepsilon})$ não for extensão galoisiana, então considere $\mathbb{L}$ o fecho galoisiano de $C_{n}(\sqrt{\varepsilon})$. Do fato de $C_{n}^{\prime}$ ser um subcorpo totalmente real e $\varepsilon$ ser uma unidade totalmente positiva, se $\tau$ é um monomorfismo de $\mathbb{L}$ em $\overline{\mathbb{Q}}$ que fixa $\mathbb{Q}(\overline{\mathbb{Q}}$ é o fecho algébrico de $\mathbb{Q}$ ), então $\tau\left(C_{n}\right) \subset C_{n}^{\prime} \mathrm{e}$

$$
\tau(\sqrt{\varepsilon})^{2}=\tau(\varepsilon) \Rightarrow \tau(\sqrt{\varepsilon})= \pm \sqrt{\tau(\varepsilon)} .
$$

Como e é uma unidade totalmente positiva,

$$
\tau(\varepsilon)>0
$$

portanto

$$
\tau\left(C_{n}(\sqrt{\Xi})\right) \subseteq \mathbb{R}
$$

Como o compositum de corpos que não ramificam fora de 2 , é um corpo que não ramifica fora de 2 , concluímos que $\mathbb{L}$ é uma extensão totalmente real que não ramifica fora de 2 . 
Portanto, pelo lema anterior (3.1.3),

$$
\mathbb{L}=C_{n+1},
$$

o que implica que a fatoração de (2) em $\mathbb{L}$ é $\lambda^{2^{n}}$ ( $\lambda$ é o único ideal primo em $\mathbb{L}$ que está sobre 2) que é a mesma fatoração de (2) em $C_{n}$, o que é absurdo, pois isso implica que em $C_{n+1}$ e em $C_{n}$, (2) tem a mesma fatoração e em $C_{n+1}$, a fatoração de (2) é da forma $\nu^{2^{n+1}}$, onde $\nu$ é o único ideal primo acima de (2) em $C_{n+1}^{\prime}$.

Logo, $\sqrt{\varepsilon} \in C_{n} \quad \forall \varepsilon \in C_{n}$, unidade totalmente positiva, ou seja, aqui está demonstrado que $U^{+} \subseteq U^{2}$. Como $U^{2} \subseteq U^{+}$, segue

$$
U^{+}=U^{2}
$$

3.3.5. Corolário. Sejam $\ell$ um primo, $m=\ell^{n}, m \geq 3, G=\operatorname{Gal}\left(\mathbb{Q}^{\prime} / \mathbb{Q}\right)$. Seja $\eta$ uma unidade em $\mathbb{Q}^{\prime}$ satisfazendo a condição:

$$
\sigma(\eta)=\eta^{r(\sigma)} \tilde{\varepsilon}(\sigma)^{m} \quad \forall \sigma \in G, \varepsilon(\sigma) \in \mathbb{Q}^{\prime} .
$$

Então existem uma raiz $m$-ésima da unidade $\zeta$ e uma unidade em $\mathbb{Q}^{\prime}$, $\varepsilon$, tal que $\eta=\zeta \xi^{m}$.

Demonstração: Inicialmente, todos esses resultados são gerais, isto é, valem para todo $\ell>1$. Sejam $\left(\mathbb{Q}^{\prime}\right)^{+}:=\mathbb{Q}\left(\zeta_{m}+\zeta_{m}^{-1}\right), U \circ$ grupo das unidades de $\mathbb{Q}^{\prime}, U^{+}$o grupo das unidades em $\mathbb{Q}^{\prime+}$. Pelo lema 3.3.2,

$$
U^{m}=\left(U^{+}\right)^{m}, \eta=\zeta_{1} \varepsilon^{+},
$$

onde $\zeta_{1}^{m}=1, \varepsilon^{+} \in U^{+}$.

Tomemos $\tau$ a conjugação complexa e consideremos a classe de $\eta$ módulo $\left\langle\zeta_{m}\right\rangle L^{T^{m}}$ $\left(\left\langle\zeta_{m}\right\rangle U^{m}\right.$ é o subgrupo gerado por $\zeta_{m}$ e $U^{m}$ contido em $\left.U\right)$.

Um representante da classe de $\eta$ é $\varepsilon^{+}$, que é invariante pela ação de $\tau$ e portanto toda a classe definida por $\eta$ é invariante pela ação de $\tau$.

Por outro lado, $r(\tau) \equiv-1(\bmod m)$ e por hipótese,

$$
\eta \equiv \tau(\eta) \equiv \eta^{m-1} \equiv \eta^{-1}\left(\bmod \left\langle\zeta_{m}\right\rangle\left(U^{+}\right)^{m}\right)
$$

Logo

$$
\eta^{2} \equiv 1\left(\bmod \left\langle\zeta_{m}\right\rangle\left(U^{+}\right)^{m}\right),
$$

ou seja, existe uma raiz $m$-ésima da unidade $\zeta_{2}$ e uma unidade $\varepsilon_{2}^{+}$em $U^{+}$tais que

$$
\eta^{2}=\zeta_{2}\left(\varepsilon_{2}^{+}\right)^{m}, \text { onde } \zeta_{2}^{m}=1 .
$$

Agora temos dois casos distintos, a considerar, o caso $\ell \neq 2$ e o caso $\ell=2$.

Caso $C \neq 2$, então temos que

$$
\eta=\zeta_{3}\left(\varepsilon_{3}^{+}\right)^{m} .
$$


para alguma raiz $m$-ésima da unidade $\zeta_{3}$ e alguma unidade $\varepsilon_{3}^{+} \in U^{+}$, pois neste caso, em $(\star), \zeta_{2}$ é um quadrado e $\varepsilon_{2}^{+}$também, porque,

$$
\left(\varepsilon_{2}^{+}\right)^{m}=\eta^{2} \zeta_{3}^{2} .
$$

com $m$ ímpar, ou seja, $\varepsilon_{2}^{+}$é um quadrado.

Caso $\ell=2$. De $(*)$, temos:

$$
\eta^{2}=\zeta_{2}\left(\varepsilon_{2}^{+}\right)^{m},
$$

$\operatorname{com} \zeta_{2}^{m}=1 \mathrm{e}$

$$
\varepsilon_{2}^{+} \in U^{+} \Rightarrow \zeta_{2}=\eta^{2}\left(\varepsilon_{2}^{+}\right)^{-m}
$$

o que mostra que $\zeta_{2}$ é um quadrado em $\mathbb{Q}^{\prime}$. Logo existe $\zeta^{\prime} \in \mathbb{Q}^{\prime}$ tal que $\left(\zeta^{\prime}\right)^{2}=\zeta_{2}$ de onde segue o resultado. 


\section{Capítulo 4}

\section{O Teorema de Kronecker-Weber}

Neste capítulo, serão apresentadas duas demonstrações do teorema de KroneckerWeber. O capítulo tem duas seções, a primeira reservada à demonstração do caso quadrático e a do teorema que demonstra que se $\mathbb{L}^{\prime} / \mathbb{Q}$ for uma extensão central sobre $\mathbb{Q}^{\prime} / \mathbb{Q}$ então $\mathbb{L}^{\prime}$ é uma extensão ciclotômica.

\subsection{O teorema chave}

4.1.1. Teorema. Toda extensão quadrática de $\mathbb{Q}, \mathbb{Q}(\sqrt{\delta})$, onde $\delta \in \mathbb{Q}$, é um corpo ciclotômico.

Demonstração: Notemos que um corpo quadrático, $\mathbb{Q}(\sqrt{\gamma})$, em que $\gamma \in \mathbb{Q}^{*}$, pode ser escrito como $\mathbb{Q}(\sqrt{d})$ onde $d \in \mathbb{Z}$ e $d$ livre de quadrados.

Pelo fato de $d$ ser inteiro racional livre de quadrados, pelo teorema fundamental da aritmética,

$$
d= \pm \ell_{1} \ell_{2} \cdots \ell_{n}
$$

onde cada $\ell_{i}$ é primo positivo e $\ell_{i} \neq \ell_{j}$ se $i \neq j$.

Logo $\mathbb{Q}(\sqrt{d})$ está contido no compositum dos corpos $\mathbb{Q}(\sqrt{-1})$ e $\mathbb{Q}\left(\sqrt{\ell_{i}}\right) \quad 1 \leq i \leq n$.

No caso $\ell \neq 2,\left(\mathbb{Z} /(\mathbb{Z})^{*}\right.$ é um subgrupo cíclico de ordem $\varphi(\ell)=\ell-1 \equiv 0(\bmod 2)$, existe um único subgrupo $U$, de índice 2 , isomorfo ao grupo dos quadrados módulo $C$.

Logo, $\mathbb{Q}\left(\zeta_{\ell}\right)$ possui exatamente um subcorpo quadrático $K_{\ell}$, o corpo fixo por $U$.

Denotaremos $\bar{a}$ a classe de resíduos de a módulo $\ell$. Para determinar $K_{\ell}$, consideremos a seguinte construção: 
Seja $\pi=\sum_{\bar{a} \neq \overline{0}} \zeta_{i}^{a}\left(\frac{a}{\ell}\right)$, onde ( é um primo que divide $d$ e $\left(\frac{a}{\ell}\right)$ é o símbolo de Legendre. Como $\pi$ é combinação inteira de números que são inteiros algébricos, segue que $\pi$ é inteiro algébrico.

Para mostrar que $\pi$ não é um quadrado nem é nulo, basta mostrar que $\pi^{2}=\ell$ ou que $\pi^{2}=-C$.

$$
\begin{gathered}
\pi^{2}=\sum_{\bar{a} \neq \overline{0}}\left(\frac{a}{\ell}\right) \zeta_{\ell}^{a} \sum_{\bar{a} \neq \overline{0}}\left(\frac{a}{\ell}\right) \zeta_{\ell}^{a} \\
=\sum_{\bar{a}, \bar{b} \neq \overline{0}}\left(\frac{a}{\ell}\right)\left(\frac{b}{\ell}\right) \zeta_{\ell}^{a+b}=\sum_{\bar{a}, \bar{b} \neq \overline{0}}\left(\frac{a b}{\ell}\right) \zeta_{\ell}^{a+b}
\end{gathered}
$$

Como $b \neq 0$ e $\ell$ é um primo, existe $a^{\prime} \in \mathbb{F}_{\ell}^{*}$ tal que $a \equiv a^{\prime} b(\bmod \ell)$. Assim, o somatório fica:

$$
\pi^{2}=\sum_{\bar{a}^{\prime}, \bar{b} \neq \overline{0}}\left(\frac{a^{\prime} b^{2}}{\ell}\right) \zeta_{\ell}^{\left(a^{\prime}+1\right) b}=\sum_{\bar{a}^{\prime} \neq \overline{0}}\left(\frac{a^{\prime}}{\ell}\right) \sum_{\bar{b} \neq \overline{0}} \zeta_{\ell}^{\left(a^{\prime}+1\right) b} .
$$

Porém

$$
\sum_{\bar{b}^{\prime} \neq \overline{0}} \zeta_{\ell}^{\left(a^{\prime}+1\right) b}=\left\{\begin{array}{cc}
\ell-1 & \text { para } a^{\prime} \equiv-1(\bmod \ell) \\
-1 & \text { para } a^{\prime} \neq \equiv-1(\bmod \ell)
\end{array}\right.
$$

Portanto, $\pi^{2}=\left(\frac{-1}{\ell}\right) \ell-\sum_{\bar{a} \neq \overline{0}}\left(\frac{a}{\ell}\right)$.

Como há exatamente $\frac{\ell-1}{2}$ resíduos quadráticos e $\frac{\ell-1}{2}$ resíduos não quadráticos módulo $\ell$,

$$
\sum_{\bar{a} \neq \overline{0}}\left(\frac{a}{\ell}\right)=0
$$

de onde

$$
\pi^{2}=\left(\frac{-1}{\ell}\right) \ell
$$

É possível que $\pi^{2}=-\ell$, quando $\ell \equiv 1(\bmod 4)$.

Se isso ocorrer, podemos considerar $K_{\ell} \subset \mathbb{Q}\left(i, \zeta_{\ell}\right) \subset \mathbb{Q}\left(\zeta_{4 \ell}\right)$.

Agora resta mostrar que $\mathbb{Q}(\sqrt{2})$ também é ciclotômico. De fato,

$$
\mathbb{Q}\left(\zeta_{8}\right)=\mathbb{Q}(\sqrt{2} / 2+i \sqrt{2} / 2)=\mathbb{Q}(\sqrt{2}, i) \supset \mathbb{Q}(\sqrt{2}) \text {. }
$$

Portanto todos os corpos quadráticos são ciclotômicos.

Demonstraremos um teorema chave, sobre extensões cíclicas, de grau $\ell^{n}$ onde $\ell$ é um primo, de corpos ciclotômicos. 
4.1.2. Teorema. Sejam ( um primo, $m=\left(^{n}, \operatorname{com} n \geq 1, \zeta \mathrm{m}\right.$ uma raiz $m$-ésima primitiva da unidade, $G=\operatorname{Gal}\left(\mathbb{Q}\left(\zeta_{m}\right) / \mathbb{Q}\right), \Delta=\sum_{\sigma \in G} r\left(\sigma^{-1}\right) \sigma$ e $\uparrow \in \mathbb{Q}\left(\zeta_{m}\right), \hat{\imath} \neq 0$. Então

$$
\mathbb{Q}\left(\zeta_{m}\right)(\sqrt[m]{\Delta(\vartheta)})
$$

é ciclotômico.

Demonstração: $O$ caso $C=2, m=2$, é o caso quadrático, já demonstrado, então podemos supor $m \geq 3$.

Sejam

$$
\mathbb{Q}^{\prime}=\mathbb{Q}\left(\zeta_{m}\right) \quad \text { e } \quad \mathbb{L}^{\prime}=\mathbb{Q}^{\prime}(\sqrt[m]{\Delta(\gamma)})
$$

então, pelo lema 2.1.5, $\mathbb{L}^{\prime} / \mathbb{Q}$ é extensão galoisiana e $\operatorname{Gal}\left(\mathbb{L}^{\prime} / \mathbb{Q}^{\prime}\right)$ está contido no centro de $\operatorname{Gal}\left(\mathbb{L}^{\prime} / \mathbb{Q}\right)$.

4.1.2.1. Lema. Se $p \nmid m$, podemos supor que $\sum_{\sigma \in D_{\mathfrak{\beta}}} r(\sigma) \equiv 0(\bmod m) \forall \mathfrak{P}$ ideal primo que está sobre de $p$, em $\mathbb{Q}^{\prime}$.

Demonstração: Tomemos um primo $p \in \mathbb{Z}$ tal que $p \nmid m$, então, pela lei de decomposição, ${ }^{[19]}$ teremos

$$
(p)=p_{1} \cdots p_{g}
$$

Temos ainda,

$$
p^{f} \equiv 1(\bmod m)
$$

e $f$ é o menor número natural positivo tal que $p^{f} \equiv 1(\bmod m)$.

Assim, se $\mathfrak{p}$ for um primo acima de $(p)$ e $D_{\mathfrak{p}}$ for o grupo de decomposição de $(p)$ em $\mathbb{Q}^{\prime}$ temos

$$
r\left(D_{\mathfrak{p}}\right)=\left\{p^{\alpha}(\bmod m) \mid 0 \leq \alpha \leq f-1\right\},
$$

onde $r\left(D_{\mathfrak{p}}\right)=\left\{r(\sigma) \mid \sigma \in D_{\mathrm{p}}\right\}$. $^{[20]}$

- Se $\ell \neq 2$ e $p \not \equiv 1(\bmod \ell)$, então dessa igualdade, segue que

$$
\sum_{\sigma \in D_{\mathfrak{p}}} r(\sigma) \equiv \frac{p^{f}-1}{p-1}(\bmod m) \equiv 0(\bmod m) \text {. }
$$

- Se $\ell=2$ e $p \equiv 3(\bmod 4)$, então há dois subcasos a se considerar: quando $m-1 \in$ $r\left(D_{\mathrm{p}}\right)$ e quando $m-1 \notin r\left(D_{\mathrm{p}}\right)$.

• Caso $m-1 \in r\left(D_{\mathfrak{p}}\right)$, então $\sum_{\sigma \in D_{\mathfrak{p}}} r(\sigma) \equiv 0(\bmod m)$.

• Caso $m-1 \notin r\left(D_{\mathrm{p}}\right)$, então as classes laterais de $r\left(\operatorname{Gal}\left(\mathbb{Q}^{\prime} / \mathbb{Q}\right)\right) / r\left(D_{\mathrm{p}}\right)$ são da forma

$$
\left\{\overline{1}, \overline{-1}, \overline{x_{1}}, \overline{-x_{1}}, \ldots \overline{x_{t}}, \overline{-x_{t}}\right\},
$$

[19] Cf. Capítulo 1, teorema 1.2.55.

[20] $r: \operatorname{Gal}\left(\mathbb{Q}^{\prime} / \mathbb{Q}\right) \longrightarrow(\mathbb{Z} / m \mathbb{Z})^{*}$ é a aplicação que satisfaz $r(\sigma)=\zeta_{m}^{r(\sigma)} \forall \sigma \in \operatorname{Gal}\left(\mathbb{Q}^{\prime} / \mathbb{Q}\right)$. 
onde $\left\{1,-1, x_{1},-x_{1} \ldots \ldots x_{t},-x_{1}\right\}$ forma um conjunto de representantes das classes laterais de $r\left(D_{\mathrm{p}}\right)$ em $r\left(\operatorname{Gal}\left(\mathbb{Q}^{\prime} / \mathbb{Q}\right)\right)^{[21]}$. Logo

$$
\left.\operatorname{Gal}\left(\mathbb{Q}^{\prime} / \mathbb{Q}\right)=\bigcup\left(x_{i} D_{\mathrm{p}} \cup-x_{i} D_{\mathrm{p}}\right)\right)
$$

Calculando $(\Delta(\hat{i}))$, temos $(\Delta(\gamma))=(\Delta(a))\left(\Delta\left(a^{\prime}\right)\right)$ onde $a \cdot a^{\prime} \in \mathbb{Q}^{\prime}$ e $\left(\Delta\left(a^{\prime}\right)\right)$ é composto apenas por ideais primos que não aparecem na fatoração de $(p)$ em $\mathbb{Q}^{\prime}$. Assim, podemos analisar apenas os primos que estão sobre $p$, isto é, basta analisar apenas $\Delta(a)$. Dessa forma, temos

$$
(\Delta(a))=\prod_{\sigma \in G}\left(\sigma^{-1}(\mathfrak{p})\right)^{r(\sigma)} .
$$

Lembrando que o grupo de Galois de $\mathbb{Q}^{\prime} / \mathbb{Q}$ age transitivamente sobre os primos que estão acima de $(p)^{[22]}$, temos

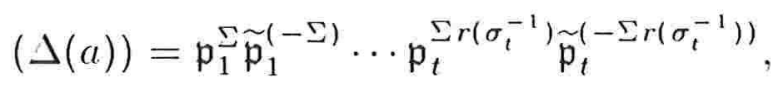

onde $\tilde{\mathfrak{p}}_{i}$ é o conjugado do primo $\mathfrak{p}_{i}, \sigma_{j}$ é um elemento do grupo de Galois de $\mathbb{Q}^{\prime} / \mathbb{Q}$ que leva o ideal primo $\mathfrak{p}_{1}:=\mathfrak{p}$ no ideal primo $\mathfrak{p}_{j}{ }^{[23]} \mathrm{e}$

$$
\Sigma=\sum_{\sigma \in D_{\mathbf{p}}} r(\sigma)
$$

Vamos mostrar que $r\left(\sigma_{t}^{-1}\right) \Sigma \equiv \Sigma(\bmod m)$. Antes precisamos mostrar que $2^{n-1} \mid \Sigma$. De fato, sabemos que $p^{f} \equiv 1\left(\bmod 2^{n}\right)$ e também sabemos, pela lei de decomposição[24], que

$$
\sum_{\sigma \in D_{\mathrm{p}}} r(\sigma) \equiv \sum_{k=1}^{f-1} p^{k}\left(\bmod 2^{n}\right) .
$$

Multiplicamos ambos os membros por $p$ e subtraímos a equivalência original, obtendo a seguinte equivalência:

$$
(p-1) \sum_{\sigma \in D_{\mathrm{p}}} r(\sigma) \equiv p^{f}-1\left(\bmod 2^{n}\right)
$$

[21] Notemos que existe um número par de classes laterais, pois $\ell=2$ e podem ser tomados da forma, $x,-x$, justamente porque a classe de $m-1$ não pertence a $r\left(D_{\mathrm{p}}\right)$.

[22] Cf. Capítulo 1, teorema 1.2.21.

[23] Isso é possível, pois o grupo de Galois de $\mathbb{Q}^{\prime}$ sobre $\mathbb{Q}$ age transitivamente sobre os primos que estão sobre $(p)$ em $\mathbb{Q}^{\prime}$. Cf. Capítulo 1, teorema 1.2.21.

[24] Cf. Capítulo 1, teorema 1.2.55. 
Então temos que $2^{n} \mid(p-1) \sum_{\sigma \in D_{p}} r(\sigma)$. Usando a hipótese de $p$ ser congruente a 3 módulo 4 , temos que $p-1$ é congruente a 2 módulo 4 . Portanto temos que

$$
2^{n-1} \mid \sum_{\sigma \in D_{\mathrm{p}}} r(\sigma) .
$$

Observemos que $2 \nmid r\left(\sigma_{j}\right)$, pois $\operatorname{Gal}\left(\mathbb{Q}^{\prime} / \mathbb{Q}\right) \sim\left(\mathbb{Z} / 2^{n} \mathbb{Z}\right)^{*}$. Assim, temos

$$
\begin{gathered}
\Sigma \equiv u 2^{n-1}\left(\bmod 2^{n}\right) \Rightarrow r\left(\sigma_{j}^{-1}\right) \Sigma \equiv u r\left(\sigma_{j}^{-1}\right) 2^{n-1}\left(\bmod 2^{n}\right) \Rightarrow \\
\Rightarrow r\left(\sigma_{j}^{-1}\right) \Sigma-\Sigma \equiv u 2^{n-1}\left(r\left(\sigma_{j}^{-1}\right)-1\right)\left(\bmod 2^{n}\right) .
\end{gathered}
$$

Como $r\left(\sigma_{j}^{-1}\right)$ é ímpar, temos

$$
r\left(\sigma_{j}^{-1}\right) \Sigma-\Sigma \equiv 0\left(\bmod 2^{n}\right)
$$

ou seja,

$$
r\left(\sigma_{j}^{-1}\right) \Sigma \equiv \Sigma\left(\bmod 2^{n}\right) .
$$

Logo, $(*)$, pode ser reescrita como

$$
(\Delta(a))=\mathfrak{p}_{1}^{\Sigma} \widetilde{\mathfrak{p}}_{1}^{\Sigma} \cdots \mathfrak{p}_{t}^{\Sigma} \widetilde{\mathfrak{p}}_{t}^{\Sigma}(\delta)^{m}=\left(\mathfrak{p}_{1} \widetilde{\mathfrak{p}}_{1} \cdots \mathfrak{p}_{t} \widetilde{\mathfrak{p}}_{t}\right)^{\Sigma}(\delta)^{m}=(p)^{\Sigma}(\delta)^{m}, \quad \delta \in \mathbb{Q}^{\prime} .
$$

Mas sabemos que $\Sigma$ é múltiplo de $2^{n-1}$, portanto $\mathbb{Q}^{\prime}(\sqrt[m]{\Delta(a)})=\mathbb{Q}^{\prime}(\sqrt{p})$, que se reduz ao caso quadrático e portanto conseguimos mostrar, neste caso, $m=2^{n}$ e $m-1 \notin r\left(D_{\mathrm{p}}\right)$, que o corpo $\mathbb{Q}^{\prime}(\sqrt[m]{\Delta(a)})$ é ciclotômico.

Continuemos com a demonstração de que $\mathbb{Q}^{\prime}(\sqrt[m]{\Delta(\gamma)})$ é ciclotômico, agora, com a propriedade que, na fatoração de $(\Delta(\gamma))$ em $\mathbb{Q}^{\prime}$, só aparecem primos em que vale a propriedade

$$
\sum_{\sigma \in D_{\mathrm{p}}} r(\sigma) \equiv 0(\bmod m)
$$

pois quando $\sum_{\sigma \in D_{\mathrm{p}}} r(\sigma) \not \equiv 0(\bmod m)$, já sabemos que são ciclotômicos.

Seja $S$ o conjunto de todos os primos racionais que são congruentes a

$$
\left\{\begin{array}{l}
1(\bmod \ell) \text { para } \ell \neq 2 \\
\text { ou } \\
1(\bmod 4) \text { para } \ell=2
\end{array} .\right.
$$

O ideal principal $(\gamma)$ pode ser decomposto em três fatores primos entre si:

$$
(\gamma)=\mathfrak{a b}\left(1-\zeta_{m}\right)^{c} \quad(c \in \mathbb{Z})
$$

onde, por definição, o ideal a consiste no produto de todos os ideais primos em $\mathbb{Q}^{\prime}$ cujos primos racionais que estão abaixo de a, pertencem a $S$. 
Por definição o ideal b é o produto de ideais primos, em $\mathbb{Q}^{\prime}$, cujos ideais primos que estão abaixo de $\mathfrak{b}$, são diferentes de ( e não pertencem a $S$.

Da decomposição de $\left(\gamma_{i}\right)$, segue:

$$
(\Delta(\jmath))=\Delta(\mathfrak{a}) \Delta(\mathfrak{b})\left(\Delta\left(1-\zeta_{m}\right)\right)^{c} .
$$

De $(* *), \Delta(\mathfrak{b})$ é uma potência $m$-ésima de algum ideal $\mathfrak{b}^{\prime}$

$$
\Delta(\mathfrak{b})=\left(\mathfrak{b}^{\prime}\right)^{m}
$$

Pelo teorema de Kummer para ideais primos ${ }^{[25]}, \mathfrak{b}^{\prime}$ é equivalente ao produto, $\mathfrak{a}^{\prime}$, de ideais primos de grau 1 (e diferentes de $\left(1-\zeta_{m}\right)$ )

$$
\mathfrak{b}^{\prime}=(\beta) \mathfrak{a}^{\prime} \quad\left(\beta \in \mathbb{Q}^{\prime}\right) .
$$

Também vale a seguinte igualdade para os ideais:

$$
\left(\sigma\left(1-\zeta_{m}\right)\right)=\left(1-\zeta_{m}\right)
$$

para todo $\sigma \in G$, desse modo,

$$
\left(\Delta\left(1-\zeta_{m}\right)\right)=\left(1-\zeta_{m}\right)^{\Sigma}
$$

onde $\Sigma$ denota a soma $\sum_{\sigma \in G} r\left(\sigma^{-1}\right)$, que é divisível por $m$, pois $m \geq 3$ e a imagem de $G$ sob $r$ contém o subgrupo $\{-1,1(\bmod m)\}$.

De (1), (2), (3) e (4) deduzimos

$$
\left(\Delta(\gamma) \beta^{-m}\right)=(\Delta(\mathfrak{a}))\left(\mathfrak{a}^{\prime}\right)^{m}\left(1-\zeta_{m}\right)^{c \Sigma} .
$$

e

$$
\mathbb{L}^{\prime}=\mathbb{Q}^{\prime}(\sqrt[m]{\Delta(\gamma)})=\mathbb{Q}^{\prime}(\sqrt[m]{\Xi}),
$$

a saber, $\Xi=\Delta(\gamma) 3^{-m}\left(1-\zeta_{m}\right)^{-c \Sigma} \mathrm{e} \Xi$ satisfaz a condição

$$
(\varepsilon)=\Delta(\mathfrak{a}) \cdot\left(\mathfrak{a}^{\prime}\right)^{m} \text {. }
$$

Suponhamos, primeiramente que $m>4$.

Pelo lema 2.1.9 existem um elemento $\psi \in \mathbb{Q}^{\prime}$ e uma raiz $m$-ésima da unidade $\zeta$ tais que

$$
\mathbb{L}^{\prime}=\mathbb{Q}^{\prime}(\sqrt[m]{\varepsilon})=\mathbb{Q}^{\prime}(\sqrt[m]{\Delta(\psi) \zeta})
$$

onde $\psi$ pode ser escolhido tal que o ideal principal ( $)$ é o produto dos ideais primos de grau 1 , que aparecem na fatoração de $(\hat{i})$, em $\mathbb{Q}^{\prime}$, como produto de primos.

[25] Cf. Capítulo 1, teorema 1.2.57. 
Então o primo racional que está abaixo de $\iota$ pertence a $S$, pois todo primo que divide $\mathfrak{a}$, tem seu primo abaixo em $S$, e pelo teorema de Weber para somas de Gauss ${ }^{[26]}$, existe um elemento $\tau(p) \in \mathbb{Q}\left(\zeta_{m}, \zeta_{p}\right)=\mathbb{Q}^{\prime}\left(\zeta_{p}\right)$ tal que

$$
\omega(\mathfrak{p}):=\tau(\mathfrak{p})^{m}
$$

pertence a $\mathbb{Q}^{\prime}$ e satisfaz a condição

$$
(\omega(\mathfrak{p}))=\Delta(\mathfrak{p})
$$

Como ( $\dot{ }$ ) pode ser decomposto, em $\mathbb{Q}^{\prime}$, como produto de primos, então podemos ordenar esses primos de tal modo que os primeiros $t_{1}$ primos estejam sobre um primo $p_{1} \in \mathbb{Q}$ previamente fixado. Assim, fixemos $p_{1} \in \mathbb{Q}$; portanto temos

$$
(\psi)=\mathfrak{p}_{1_{1}}^{\alpha_{1_{1}}} \mathfrak{p}_{1_{2}}^{\alpha_{1_{2}}} \cdots \mathfrak{p}_{1_{t_{1}}}^{\alpha_{1_{1}}} \mathfrak{q}
$$

onde q é um produto de ideais primos relativamente primos com o ideal primo $\left(p_{1}\right)$ (isto é, que não estão sobre $\left.\left(p_{1}\right)\right)$ e os primos $p_{1_{i}}^{\alpha_{1}}$ são os ideais primos sobre $p_{1}$ que aparecem na decomposição de $(\psi)$ em $\mathbb{Q}^{\prime}$. Portanto, existe um $\Gamma_{\mathrm{p}} \in \mathbb{Z}[G]$ (basta tomarmos $\Gamma_{\mathrm{p}}=$ $\sum_{\substack{j=1 \\ \text { que }}}^{t_{1}} \alpha_{1_{i}} \sigma_{i}$, onde $\sigma_{i}$ leva o ideal $\mathfrak{p}_{1}$ no ideal $\left.\mathfrak{p}_{t_{i}}\right)$, e algum ideal $\mathfrak{q}$ primo com o ideal $(p)$ tal

$$
(\psi)=\Gamma_{\mathfrak{p}}(\mathfrak{p}) \cdot \mathfrak{q} .
$$

Desse modo é possível obter a decomposição:

$$
(\psi)=\prod \Gamma_{\mathbf{p}}(\mathfrak{p})
$$

onde $\mathfrak{p}$ percorre todo o conjunto dos ideais primos que estão sobre os primos racionais que pertencem a $S$ e onde os $\Gamma_{\mathrm{p}}$ pertencem a $\mathbb{Z}[G]$.

De (6), do fato de $G$ ser abeliano e da ação de $\Delta$ ser multiplicativa, temos:

$$
(\Delta(\psi))=\prod \Gamma_{\mathfrak{p}}(\Delta(\mathfrak{p}))=\prod\left(\Gamma_{\mathrm{p} w}(\mathfrak{p})\right) .
$$

Portanto o corpo dado

$$
\mathbb{L}^{\prime}=\mathbb{Q}^{\prime}\left(\sqrt[m]{\Delta(\psi) \zeta^{\prime}}\right)
$$

está contido no compositum dos corpos ciclotômicos $\mathbb{Q}\left(\zeta_{m}, \zeta_{p}\right)(p \in S)$ e algum corpo

$$
\mathbb{L}^{\prime \prime}=\mathbb{Q}^{\prime}(\sqrt[m]{\eta})
$$

onde $\eta$ é uma unidade em $\mathbb{Q}^{\prime}$, pois repete-se o processo para todos os primos, e a extensão $\mathbb{L}^{\prime \prime} / \mathbb{Q}$ é central sobre $\mathbb{L}^{\prime \prime} / \mathbb{Q}^{\prime}$.

Pelo corolário 3.3.5

$$
\eta=\zeta^{\prime} \xi^{m}
$$

onde $\zeta^{\prime}$ é alguma raiz $m$-ésima da unidade e $\xi$ é uma unidade em $\mathbb{Q}^{\prime}$. Portanto $\mathbb{L}^{\prime \prime}$ está contido em $\mathbb{Q}^{\prime}\left(\sqrt[m]{\zeta_{m}}\right)=\mathbb{Q}\left(\zeta_{m^{2}}\right)$ e portanto o corpo $\mathbb{L}^{\prime}$ é ciclotômico.

[26] Cf. Capítulo 3, teorema 3.2.5. Notemos que, para $n=1, m=\ell$ e basta usar o teorema de Kummer (cf. Capítulo 3, teorema 3.2.4). 


\subsection{O teorema de Kronecker-Weber}

A demonstração do teorema de Kronecker-Weber será feita por passos, primeiro para extensões cíclicas cujo grau é uma potência de primo.

Para mostrar que o resultado vale para extensões cíclicas de grau potência de primo, comecemos com uma extensão cíclica de grau primo. Para extensões cíclicas de grau primo, podemos tirar de duas maneiras distintas. A primeira, como aplicação direta do teorema anterior e a segunda usando um pequeno argumento sobre o grau e ramificações de primos.

4.2.1. Proposição. Sejam $\ell$ um primo e $\mathbb{L} / \mathbb{Q}$ uma extensão cíclica de grau $\ell$. Então $\mathbb{L}$ é uma extensão ciclotômica.

Demonstração: Seja $\mathbb{Q}^{\prime}=\mathbb{Q}\left(\zeta_{\ell}\right), \mathbb{L}^{\prime}=\mathbb{Q}^{\prime} \cdot \mathbb{L}$. Pelo corolário 2.1.9, existe um $\gamma \in \mathbb{Q}^{\prime}, \gamma^{\prime} \neq$ 0 , tal que

$$
\mathbb{L}^{\prime}=\mathbb{Q}^{\prime}(\sqrt[\mathfrak{l}]{\Delta(\gamma)}) .
$$

Pelo teorema 4.1.2, $\mathbb{L}^{\prime}$ é ciclotômico. Portanto de $\mathbb{L} \subseteq \mathbb{L}^{\prime}$, tiramos que $\mathbb{L}$ é ciclotômico.

4.2.2. Definição. Sejam $\ell$ um primo e $\mathbb{L} / \mathbb{K}$ uma extensão finita galoisiana de corpos de números de grau $\ell^{n}$, e sejam $\mathfrak{p} \subset \mathcal{O}_{\mathbb{E}}$ um ideal primo e $\mathfrak{P}_{1}^{e_{1}} \cdots \mathfrak{P}_{g}^{e_{g}}$ a decomposição de $\mathfrak{p} \mathcal{O}_{\mathbb{L}}$ em $\mathcal{O}_{\mathbb{L}}$. Dizemos que $\mathfrak{P}_{i}$ ramifica moderadamente, se $\operatorname{mdc}\left(\epsilon\left(\mathfrak{P}_{i} / \mathfrak{p}\right), \ell\right)=1$ e diremos que $\mathfrak{P}_{i}$ ramifica selvagemente $\operatorname{se} \operatorname{mdc}\left(\epsilon\left(\mathfrak{P}_{i} / \mathfrak{p}\right), \ell\right)=\ell$.

4.2.3. Lema. Sejam $\ell$ um primo e $\mathbb{L} / \mathbb{Q}$ uma extensão galoisiana cíclica de grau $\ell^{n}, n \geq 1$. Então existe um único corpo, $\mathbb{L}_{1}$, de grau $\ell$ sobre $\mathbb{Q}$ e $\mathbb{L}_{1}$ pode ser imerso num corpo cíclico, ciclotômico de grau $\ell^{n}$.

Demonstração: Seja $\left\{p_{1}, \ldots, p_{s}\right\}$ o conjunto de todos os primos que ramificam moderadamente na extensão $\mathbb{L}_{1} / \mathbb{Q}$ (pelos teoremas 1.2.46 e 1.2.47, existe pelo menos um primo que ramifica moderadamente na extensão $\mathbb{L}_{1} \neq \mathbb{Q}$ ).

Se algum primo $p, p \neq \ell$, ramifica em $\mathbb{L}_{1} / \mathbb{Q}$ então $p$ é totalmente e moderadamente ramificado em $\mathbb{L} / \mathbb{Q}$. De fato, tomemos um ideal primo $(p) \subset \mathbb{Q}$ e algum ideal primo $\mathfrak{P}$ sobre $(p)$ em $\mathbb{L}$ e consideremos $D_{\mathfrak{P}} \circ$ grupo de decomposição de $\mathfrak{P}$ sobre $(p)$ em $\mathbb{L}$.

Seja $\mathbb{L}^{D_{\mathfrak{l}}}$ o corpo de decomposição de $\mathfrak{P}$ contido em $\mathbb{L}$. [2 ${ }^{[2]}$

De $\mathbb{L} / \mathbb{Q}$ ser uma extensão galoisiana cíclica de grau $l^{n}$ e $\ell$ ser um primo, temos que $\mathbb{L}^{D \cdot \Re}$ tem grau $\ell^{k}(k \in \mathbb{N})$, sobre $\mathbb{Q}$.

Como $\operatorname{Gal}(\mathbb{L} / \mathbb{Q})$ é cíclico, para cada $k \geq 1$ natural, $\mathbb{L}$ só tem um subcorpo de grau $c^{k}$, e esse subcorpo contém $\mathbb{L}_{1}$. Porém, no corpo de decomposição $\mathbb{L}^{D_{\sharp 3}}$, os ideais primos acima de $(p)$, devem aparecer com índice de ramificação 1 e como o índice de ramificação é multiplicativo em relação às extensões de corpos, temos que $\mathbb{L}^{D_{\sharp \mathfrak{\beta}}}=\mathbb{Q}$, ou seja, $D_{\mathfrak{P}}=$ $\operatorname{Gal}(\mathbb{L} / \mathbb{Q})$.

[2i] Neste corpo, $\mathbb{L}^{D_{\Downarrow\}}}$, o ideal primo $(p)$ se decompõe completamente, isto é, o primo $p$ é não ramificado e tem grau $f=1$. 
Seja $T=T_{\mathfrak{P} / p}(\mathbb{L} / \mathbb{Q})$ o grupo de inércia de $\mathfrak{P}$ sobre $p$ contido em $\operatorname{Gal}(\mathbb{L} / \mathbb{Q})$ e seja $\mathbb{L}^{T}$ o subcorpo fixo por $T$ em $\mathbb{L}$. $^{[28]}$

Do fato de $p$ ser um primo que ramifica em $\mathbb{L}_{1} \subset \mathbb{L}$, do grau de $\mathbb{L}_{1} / \mathbb{Q}$ ser $\ell$, $C$ ser um primo e $\mathbb{L} / \mathbb{Q}$ ser uma extensão cíclica galoisiana de grau (", temos que $\mathbb{L}_{1}$ estará contido em $\mathbb{L}^{T}$ se $\mathbb{L}^{T} / \mathbb{Q}$ tiver grau maior que 1 , o que seria um absurdo; pois o índice de ramificação é multiplicativo em relação às extensões de corpos, em $\mathbb{L}_{1}$ o primo $p$ ramifica enquanto em $\mathbb{L}^{T}$ o primo $p$ é inerte. Logo $\mathbb{L}^{T} / \mathbb{Q}$ tem grau 1 e portanto $T=D_{\mathfrak{P}}=\operatorname{Gal}(\mathbb{L} / \mathbb{Q})$.

Portanto o índice de ramificação é $\ell^{n}$ e necessariamente divide $(p-1)^{[29]}$. Dessa forma, $p_{i} \in\left\{p_{1}, \ldots, p_{s}\right\}$ é tal que $p_{i} \equiv 1\left(\bmod \left(^{n}\right) \forall i, 1 \leq i \leq s\right.$. Cada corpo ciclotômico $\mathbb{Q}\left(\zeta_{p_{i}}\right)(1 \leq i \leq s)$, contém uma única subextensão cíclica $\mathbb{L}\left(p_{i}\right) / \mathbb{Q}$ de grau $\ell^{n}$. Mais ainda, no caso $\ell \neq 2$, escolhemos única subextensão de grau $l^{n}$ dentro de $\mathbb{Q}\left(\zeta_{\ell^{n}+1}\right)$ e no caso $\ell=2$, escolhemos a única subextensão cíclica totalmente real $\mathbb{L}(2) / \mathbb{Q}$ de grau $2^{n}$ contida no corpo $\mathbb{Q}\left(\zeta_{2^{n+2}}\right)$.

Obviamente, o corpo ciclotômico $\mathbb{L}_{1}$ está contido no compositum

$$
\mathbb{L}^{\prime}:=\mathbb{L}(\ell) \cdot \mathbb{L}\left(p_{1}\right) \cdots \mathbb{L}\left(p_{s}\right),
$$

cujo grupo de Galois, $H$, é o produto direto

$$
H=H(\ell) \times H\left(p_{1}\right) \times \cdots \times H\left(p_{s}\right)
$$

onde os subgrupos cíclicos $H(\ell), H\left(p_{1}\right), \ldots, H\left(p_{s}\right)$ são os subgrupos de inércia correspondentes aos primos $\ell$ e $p_{i}(1 \leq i \leq s)$.

Agora mostremos que é possível tomarmos apenas um subcorpo $\mathbb{F}$ de $\mathbb{L}^{\prime}$, cujo grupo de Galois é cíclico de ordem $\ell^{n}$ e que contém $\mathbb{L}_{1}$.

O epimorfismo canônico

$$
H:=\operatorname{Gal}\left(\mathbb{L}^{\prime} / \mathbb{Q}\right) \longrightarrow \operatorname{Gal}\left(\mathbb{L}_{1} / \mathbb{Q}\right)
$$

pode ser composto com o isomorfismo $\varphi: \operatorname{Gal}\left(\mathbb{L}_{1} / \mathbb{Q}\right) \longrightarrow \mathbb{Z} / \mathfrak{Z}$.

Dessa maneira, temos um epimorfismo $\widetilde{\varphi}: H \longrightarrow \mathbb{Z} /(\mathbb{Z}$. Restringindo $\widetilde{\varphi}$ aos subgrupos cíclicos $H(\ell)$ e $H\left(p_{i}\right)(1 \leq i \leq s)$, temos morfismos

$$
\begin{aligned}
\widetilde{\varphi_{\ell}}: H(\ell) & \longrightarrow \mathbb{Z} /(\mathbb{Z} \\
\widetilde{\tau_{p_{i}}}: H\left(p_{i}\right) & \longrightarrow \mathbb{Z} /(\mathbb{Z} \quad(1 \leq i \leq s)
\end{aligned} .
$$

Os morfismos $\widetilde{\varphi_{p_{i}}}(1 \leq i \leq s)$ são sobrejetivos. Seja $\mu$ o epimorfismo canônico

$$
\nu: \mathbb{Z} / \ell^{n} \mathbb{Z} \longrightarrow \mathbb{Z} / \ell \mathbb{Z}
$$

[28] Neste corpo, $\mathbb{L}^{T}$, o ideal primo $(p)$ é inerte, ou seja, qualquer primo $\mathfrak{p}$ que divida $(p)$ no anel de inteiros de $\mathcal{O}_{\mathbb{E}^{T}}$, tem índice de ramificação 1 e grau $f$.

[29] Cf. Capítulo 1, corolário 1.2.23, $f=1$ e $g=1$ e o teorema 1.2.38. 
Como todos os grupos $H(c), H\left(p_{i}\right)$ são de ordem $\ell^{n}$, os morfismos $\widetilde{\tau} \ell, \widetilde{\tau_{p}}$ podem ser estendidos a morfismos

$$
\begin{aligned}
& \widetilde{\psi^{\prime} \ell}: H(\ell) \longrightarrow \mathbb{Z} / \epsilon^{n} \mathbb{Z} . \\
& \widetilde{\mho_{p_{i}}}: H\left(p_{i}\right) \longrightarrow \mathbb{Z} / \epsilon^{n} \mathbb{Z} \quad(1 \leq i \leq s)
\end{aligned}
$$

tais que

$$
\nu \circ \widetilde{\psi_{\ell}}=\widetilde{\varphi_{\ell}} \quad \nu \circ \widetilde{\psi_{p_{i}}}=\widetilde{\varphi_{p_{i}}} \quad(1 \leq i \leq s)
$$

Os morfismos $\widetilde{v_{i}}, \widetilde{v_{i}},(1 \leq i \leq s)$ determinam um morfismo

$$
\widetilde{\psi}: H \longrightarrow \mathbb{Z} / \ell^{n} \mathbb{Z}
$$

com a propriedade $\nu \circ \tilde{\psi}=\tilde{\varphi}$.

Então $\widetilde{\psi}$ será um epimorfismo se pelo menos $\widetilde{\psi_{\ell}}$ ou $\widetilde{\psi_{p}}$ for um epimorfismo.

Note que o subcorpo, $\mathbb{M}$ de $\mathbb{L}^{\prime}$ que é fixo por $\operatorname{ker}(\widetilde{\psi})$, é uma extensão cíclica ciclotômica de grau $\ell^{n}$ contendo $\mathbb{L}_{1}$.

Portanto o lema está demonstrado.

4.2.4. Teorema (Kronecker). Sejam $\ell$ um primo, $n \geq 1, \mathbb{L} / \mathbb{Q}$ uma extensão cíclica de grau $m=\ell^{n}$ então $\mathbb{L}$ é ciclotômico.

Demonstração: Suponhamos que para toda extensão galoisiana cíclica de grau $\ell^{k}$, seja ciclotômica para algum $k \geq 1$. Tomemos uma extensão $\mathbb{L} / \mathbb{Q}$ galoisiana, cíclica de grau $\ell^{k+1}$. Então $\mathbb{L}$ contém uma subextensão $\mathbb{L}_{1}$ galoisiana, de grau $\ell$ sobre $\mathbb{Q}$. Pelo lema anterior, $\mathbb{L}_{1}$ é ciclotômico pode ser imerso num corpo ciclotômico de grau $\ell^{n}$.

Utilizando a mesma notação do lema anterior, temos como escólio, os isomorfismos canônicos:

$$
\operatorname{Gal}(\mathbb{M} / \mathbb{Q}) \longrightarrow H / \operatorname{ker}(\bar{\psi}) \longrightarrow \mathbb{Z} /\left(\ell^{k}\right) \mathbb{Z}
$$

Pelo lema 1.1.1, o corpo $\mathbb{L}$ e o corpo construído $\mathbb{M}$ diferem por um corpo $\mathbb{E}$ de grau menor ou igual a $C^{k}$ sobre $\mathbb{Q}$ e pela hipótese de indução, $\mathbb{E}$ é um corpo ciclotômico e de $\mathbb{L} \cdot \mathbb{M}=\mathbb{E} \cdot \mathbb{M}$ concluímos que $\mathbb{L}$ é ciclotômico.

Kronecker quando enunciou seu famoso teorema para extensões abelianas de $\mathbb{Q}$ provou apenas para extensões cíclicas de $\mathbb{Q}$, afirmando que a redução da demonstração do teorema para extensões abelianas a partir de extensões cíclicas seria simples. Após tê-lo enunciado, foi necessário mais algum tempo até que Kronecker realmente demonstrasse o teorema. No caso em que as extensões são cíclicas de grau $2^{k}$, Kronecker afirmou ter dificuldades com a sua demonstração.

Agora apresentaremos a demonstração do mesmo resultado feita por Weber. A diferença entre essa demonstração e a demonstração do teorema anterior é que essa é uma prova mais curta e, de certo modo, mais simples. 
4.2.5. Teorema (Weber). Seja ( um primo e $\mathbb{L} / \mathbb{Q}$ uma extensão cíclica de grau $\left(^{n}\right.$. Então $\mathbb{L} / \mathbb{Q}$ é ciclotômica.

Demonstração: $\operatorname{Sejam~}\left(\right.$ um primo $n \geq 1, \mathbb{L} / \mathbb{Q}$ uma extensão cíclica de grau $m=l^{n} \neq 4$, $\mathbb{Q}^{\prime}:=\mathbb{Q}\left(\zeta_{m}\right), G:=\operatorname{Gal}\left(\left(\mathbb{Q}^{\prime} / \mathbb{Q}\right), \mathbb{L}^{\prime}=\mathbb{Q}^{\prime} \cdot \mathbb{L}\right.$ então a extensão $\mathbb{L}^{\prime} / \mathbb{Q}$ é central sobre $\mathbb{Q}^{\prime} / \mathbb{Q}$.

Pelo lema 2.1.8, vale: $\mathbb{L}^{\prime}=\mathbb{Q}^{\prime}(\sqrt[m]{\Delta(\gamma) \zeta})$ para algum $\gamma \in \mathbb{Q}^{\prime}$ e alguma raiz $m$-ésima da unidade, $\zeta$, pelo lema 2.1 .8 a extensão $\mathbb{Q}^{\prime}(\sqrt[m]{\Delta(\hat{\imath})}) / \mathbb{Q}$ é galoisiana e central sobre $\mathbb{Q}^{\prime} / \mathbb{Q}$, portanto $\mathbb{L}$ está contida no compositum dos corpos $\mathbb{Q}^{\prime}(\sqrt[m]{\Delta(\gamma)})$ e $\mathbb{Q}^{\prime}(\sqrt[m]{\zeta})$ e o corpo $\mathbb{Q}^{\prime}(\sqrt[m]{\Delta(\gamma)})$ é ciclotômico, pelo teorema 4.1.2.

Claramente o corpo $\mathbb{Q}^{\prime}(\sqrt[m]{\zeta})$ está contido no corpo ciclotômico $\mathbb{Q}\left(\zeta_{m^{2}}\right)$, sendo portanto, ciclotômico.

No caso particular $m=4$, a imagem de $G=\operatorname{Gal}(\mathbb{Q}(\sqrt{-1}) / \mathbb{Q}$ ) sob $r$ (lembrando que $\left.\sigma\left(\zeta_{m}\right)=\zeta_{m}^{r(\sigma)} \forall \sigma \in G\right)$ é simplesmente $\{1,3(\bmod 4)\}$, desde que o subcorpo totalmente real maximal de $\mathbb{L}$ tenha grau 2 ou 4 sobre $\mathbb{Q}$.

$O$ corpo $\mathbb{Q}(\sqrt{-1})$ não pode estar contido em $\mathbb{L}$, pois $\mathbb{L}$ é totalmente real, por esta razão, $\mathbb{L} \cap \mathbb{Q}(\sqrt{-1})=\mathbb{Q}$ e a extensão de grupos

$$
\{1\} \longrightarrow \operatorname{Gal}\left(\mathbb{L}^{\prime} / \mathbb{Q}^{\prime}\right) \longrightarrow \operatorname{Gal}\left(\mathbb{L}^{\prime} / \mathbb{Q}\right) \longrightarrow \operatorname{Gal}\left(\mathbb{Q}^{\prime} / \mathbb{Q}\right) \longrightarrow\{1\}
$$

é central e cinde, pelo lema 2.1.7, existe um,$\in \mathbb{Q}(\sqrt{-1})$, tal que

$$
\mathbb{L}^{\prime}=\mathbb{Q}(\sqrt{-1})\left(\sqrt[4]{\Delta(\gamma) d^{2}}\right)
$$

com $d \in \mathbb{Q}$. $\mathbb{L}^{\prime}$ está contido no compositum dos corpos ciclotômicos

$$
\mathbb{Q}(\sqrt{-1})(\sqrt[4]{\Delta(\gamma)})
$$

que, pelo teorema 4.1.2, são ciclotômicos. Portanto concluímos que $\mathbb{L}$ é ciclotômico.

No que se segue, apresentaremos uma demonstração de um resultado atualmente conhecido como teorema de Kronecker-Weber.

4.2.6. Teorema (Kronecker-Weber). Todas extensões abelianas de $\mathbb{Q}$ são ciclotômicas.

Demonstração: Seja $\mathbb{L} / \mathbb{Q}$ a extensão abeliana de $\mathbb{Q}$. Seja $G$ o grupo de Galois de $\mathbb{L} / \mathbb{Q}$ que, por ser abeliano, pode ser decomposto como produto de grupos cíclicos $G_{1} \cdot G_{2} \cdot G_{3} \cdots G_{k}$, onde cada $G_{i}$ tem ordem $p_{i}^{m_{i}}$ ( $p_{i}$ é um primo).

Sejam $\mathbb{L}_{j}$ o corpo fixo por $G_{1} \cdots \widehat{G_{j}} \cdots G_{k}$ onde $G_{1} \cdots \widehat{G_{j}} \cdots G_{k}$ significa o produto dos grupos $G_{1}, \ldots, G_{k}$ exceto o grupo $G_{j}$. Pelo teorema fundamental da teoria de Galois, $\mathbb{L}_{j} / \mathbb{Q}$ é extensão galoisiana cíclica, cujo grau é $p_{j}^{m_{j}}$. Assim, caso $p_{j}$ seja 2 , temos que $\mathbb{L}_{j} / \mathbb{Q}(i)$ é uma extensão cíclica, caso $p_{j}$ não seja 2 , então $\mathbb{L}_{j} / \mathbb{Q}$ é extensão cíclica, de qualquer modo, cada $\mathbb{L}_{j}$ em e $\mathbb{L}=\mathbb{L}_{1} \cdot \mathbb{L}_{2} \cdots \mathbb{L}_{k}$ é um corpo ciclotômico. ${ }^{[30]}$ Portanto, $\mathbb{L}$ está contido num compositum de corpos ciclotômicos, o que mostra que $\mathbb{L}$ é um corpo ciclotômico.

[30] Cf. teorema 4.2.4 de Kronecker ou o teorema 4.2.5 de Weber. 


\section{Bibliografia}

[1] Artin, E. Galois Theory, Notre Dame Math. Lectures, Number 2, 1942.

[2] Cohn, H. A Classical Invitation to Algebraic Numbers and Class Fields, SpringerVerlag, 1978.

[3] Endler, O. Teoria dos Números Algébricos, Rio de Janeiro, IMPA, 1986.

[4] Frölich, A. \& Cassels. J. W. S. Algebraic Number Theory, Academic Press, $2 \frac{n d}{}$ edition, 1967.

[5] Frölich, a \& TaYlor. M. J. Algebraic Number Theory, Cambridge University Press, 1 $\stackrel{\text { st }}{\text { pub., } 1991 .}$

[6] Goldstein, L. J. Analytic Number Theory, Prentice-Hall Inc., Englewood Cliffs, New Jersey, 1971.

[7] Gorestein, D. Finite Groups, Harper \& Row Publishers, 1968.

[8] Hasse, H. Number theory, Springer-Verlag, 3럼 edition 1969.

[9] Hilbert, D. Théorie des Corps de Nombres Algébriques, Librairie Scientifique A. Hermann et Fils, Paris, 1913.

[10] Hungerford, T. W. Algebra, Springer-Verlag, New York, 1974.

[11] IYANAGA, S. The theory of numbers, North-Holland mathematical library, v. 8, 1906.

[12] Jacobson, N. Basic Algebra I, W. H. Freeman \& Co., $2 \frac{\text { nd }}{2}$ edition, 1985.

[13] Johnson, D. L. Presentation of Groups, London Mathematical Society, Cambridge University Press, 1976.

[14] Karpilovsky, G. Field Theory (Classical Foundations and Multplicative Groups), Pure and Applied Mathematics, Marcel Dekker Inc., New York, 1988.

[15] LANG, S. Algebra, Addison Wesley Series in Mathematics, 1965.

[16] Marcus, D. A. Number Fields, Springer-Verlag, New York Inc. 1977.

[17] Neumann, O. Two Proofs of Kronecker-Weber Theorem "according to Kronecker, and Weber", J. Reine Angew. Math., 323 (1981), 105-126.

[18] Rosen, M. \& Ireland K. A Classical Introduction to Modern Number Theory, Springer-Verlag New York, 1982.

[19] Serre. J. P. Local Fields, Springer-Verlag, New York, 1979.

[20] Suzuki, M. Group Theory I, Springer-Verlag, New York, 1982. 
[21] Washington. L. C. Introduction to Cyclotomic Fields, Springer-Verlag New York, 1982.

[22] WeBER, H Theorie der Abel'schen Zahlkörper I: Abel'sche Körper; II: Über die Anzahl der Idealklassen und die Einheiten in den Kreiskörpern, deren Ordnung eine Potenz von 2 ist; III: Der Kronecker'sche Satz, Acta Math., 8, 1886, pp. 193-263. 